\title{
RERTR-10 Irradiation Summary Report
}

D. M. Perez

M. A. Lillo

G. S. Chang

G. A. Roth

N. E. Woolstenhulme

D. M. Wachs

May 2011

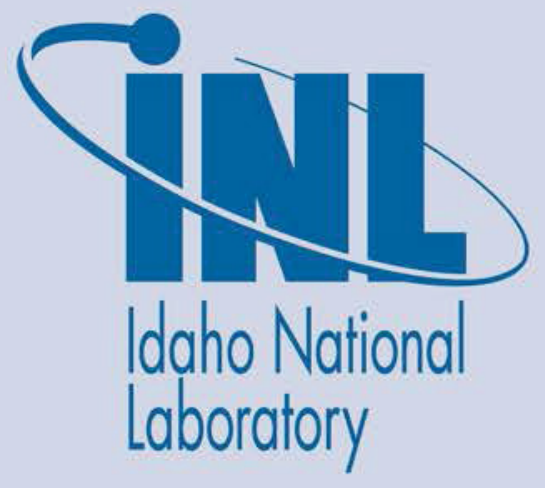

The INL is a U.S. Department of Energy National Laboratory operated by Battelle Energy Alliance 


\section{DISCLAIMER}

This information was prepared as an account of work sponsored by an agency of the U.S. Government. Neither the U.S. Government nor any agency thereof, nor any of their employees, makes any warranty, expressed or implied, or assumes any legal liability or responsibility for the accuracy, completeness, or usefulness, of any information, apparatus, product, or process disclosed, or represents that its use would not infringe privately owned rights. References herein to any specific commercial product, process, or service by trade name, trade mark, manufacturer, or otherwise, does not necessarily constitute or imply its endorsement, recommendation, or favoring by the U.S. Government or any agency thereof. The views and opinions of authors expressed herein do not necessarily state or reflect those of the U.S. Government or any agency thereof. 
INL/EXT-10-18456

\title{
RERTR-10 Irradiation Summary Report
}

\author{
D. M. Perez \\ M. A. Lillo \\ G. S. Chang \\ G. A. Roth \\ N. E. Woolstenhulme \\ D. M. Wachs
}

May 2011

\section{Idaho National Laboratory \\ Idaho Falls, Idaho 83415}

http://www.inl.gov

Prepared for the

U.S. Department of Energy

Office of National Nuclear Security Administration

Under DOE Idaho Operations Office

Contract DE-AC07-05ID14517 


\title{
RERTR-10 Irradiation Summary Report
}

\author{
INL/EXT-10-18456
}

Revision 0

May 2011

Approved by:

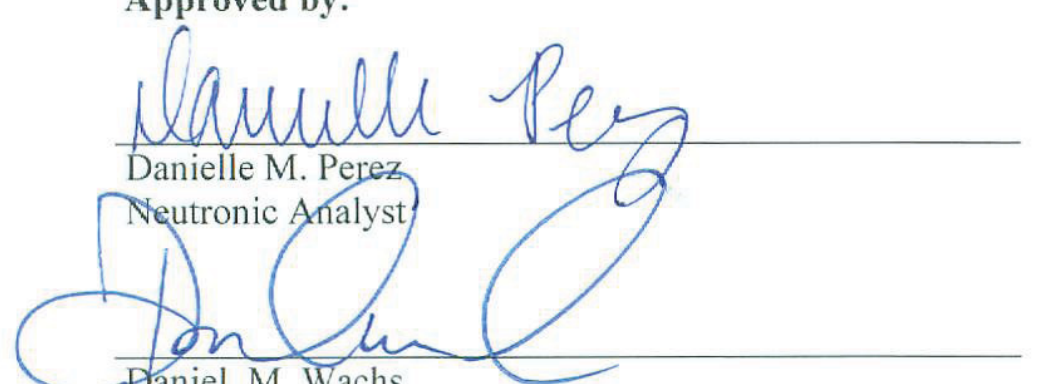

Baniel. M. Wachs

Principle Investigator
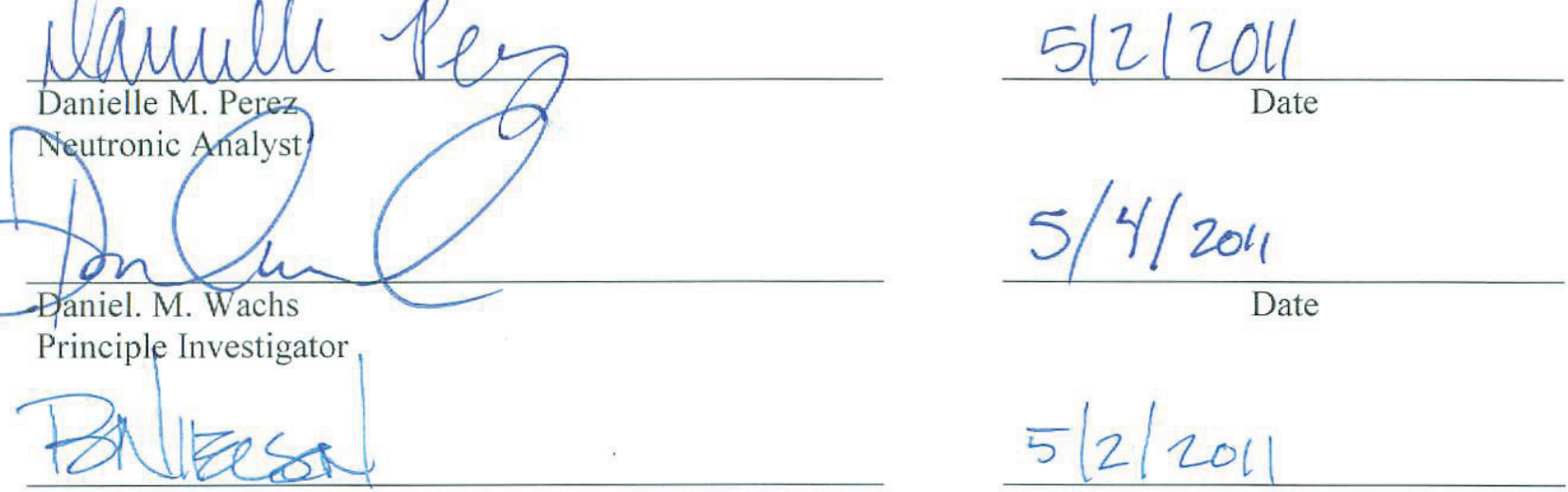

Bruce Nielson

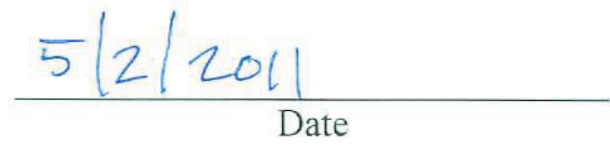

Experiment Manager 


\section{SUMMARY}

The Reduced Enrichment for Research and Test Reactor (RERTR) experiment RERTR-10 was designed to further test the effectiveness of modified fuel/clad interfaces in monolithic fuel plates. The experiment was conducted in two campaigns: RERTR-10A and RERTR-10B. The fuel plates tested in RERTR-10A were all fabricated by Hot Isostatic Pressing (HIP) and were designed to evaluate the effect of various Si levels in the interlayer and the thickness of the $\mathrm{Zr}$ interlayer (0.001") using 0.010 " and 0.020 " nominal foil thicknesses. The fuel plates in RERTR-10B were fabricated by Friction Bonding (FB) with two different thickness Si layers and $\mathrm{Nb}$ and $\mathrm{Zr}$ diffusion barriers. ${ }^{1}$

The following report summarizes the life of the RERTR-10A/B experiment through end of irradiation, including as-run neutronic analysis results, thermal analysis results and hydraulic testing results. 


\section{CONTENTS}

SUMMARY

ACRONYMS iv

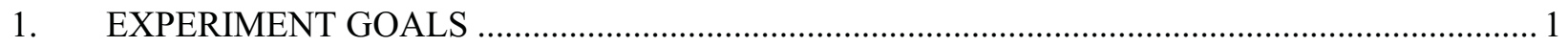

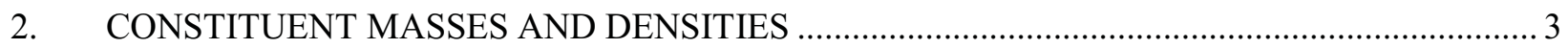

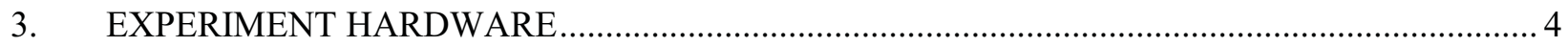

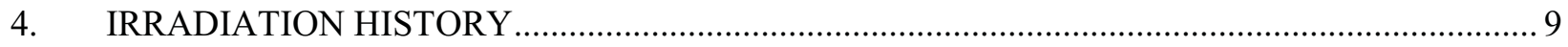

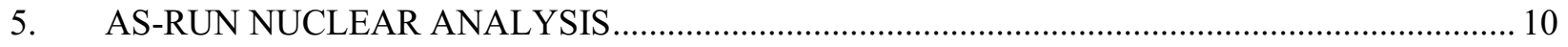

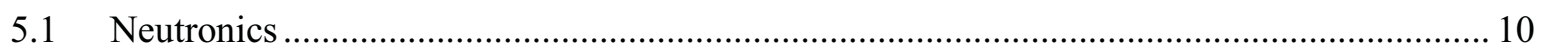

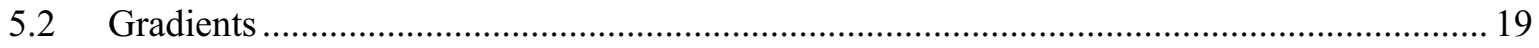

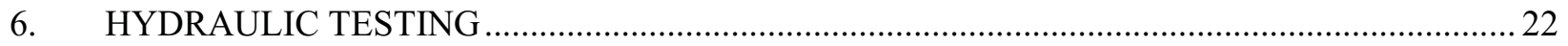

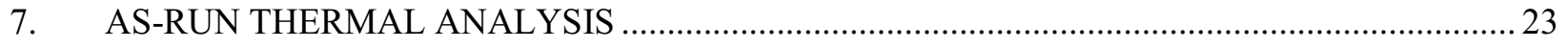

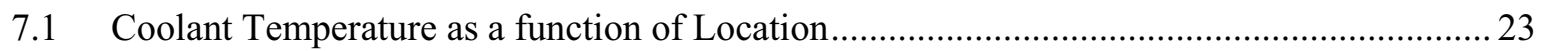

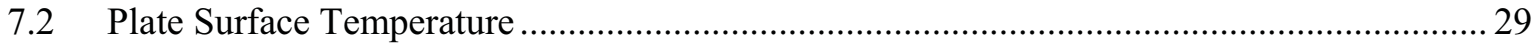

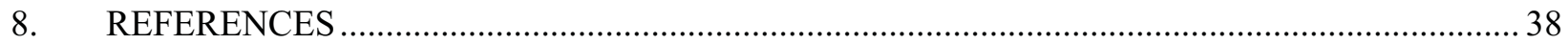

Appendix A Individual Plate Power and Fission Density Plots.......................................................... 39

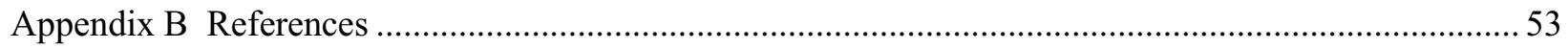

FIGURES

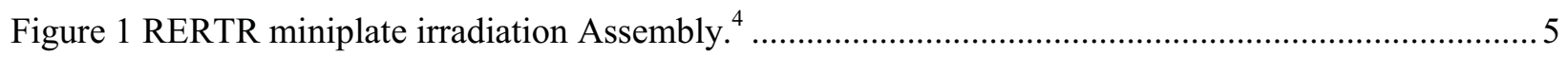

Figure 2. DWG-630244: RERTR monolithic fuel miniplate.............................................................. 6

Figure 3. DWG-630245: RERTR thick (0.020 in) monolithic fuel miniplate. .......................................... 7

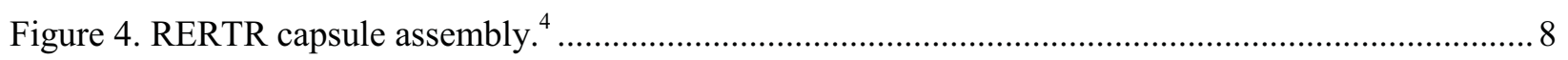

Figure 5. RERTR-10 fuel meat thermal neutron flux L2ARs in transverse direction. ${ }^{6}$............................. 19

Figure 6. RERTR-10 fuel meat fission rate L2ARs in transverse direction. ${ }^{6}$......................................... 20

Figure 7. RERTR-10 fuel meat thermal neutron flux L2ARs in axial direction. ${ }^{6}$................................... 20

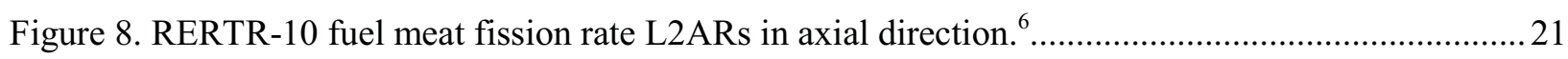

Figure 9. Coolant temperature as a function of location along the test assembly for Cycle 142B 01 (BOC). ${ }^{9}$.

Figure 10. Coolant temperature as a function of location along the test assembly for Cycle 142B 02. ${ }^{9}$

Figure 11. Coolant temperature as a function of location along the test assembly for Cycle 142B 03. ${ }^{9}$ 
Figure 12. Coolant temperature as a function of location along the test assembly for Cycle 142B 04 (EOC). ${ }^{9}$

Figure 13. Coolant temperature as a function of location along the test assembly for Cycle 143A 01 (BOC). ${ }^{9}$

Figure 14. Coolant temperature as a function of location along the test assembly for Cycle 143A $02 .^{9}$ 26

Figure 15. Coolant temperature as a function of location along the test assembly for Cycle 143A 03. ${ }^{9}$

Figure 16. Coolant temperature as a function of location along the test assembly for Cycle 143A 04. ${ }^{9}$

Figure 17. Coolant temperature as a function of location along the test assembly for Cycle 143A 05 (EOC). ${ }^{9}$

\section{TABLES}

Table 2. RERTR-10A/B constituent masses and densities. ${ }^{2,3}$............................................................. 3

Table 3. RERTR-10 Irradiation Hardware Drawing List. ${ }^{4}$.................................................................... 4

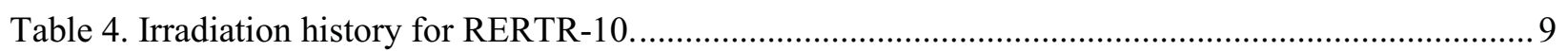

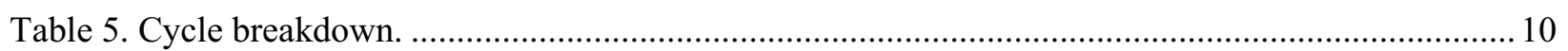

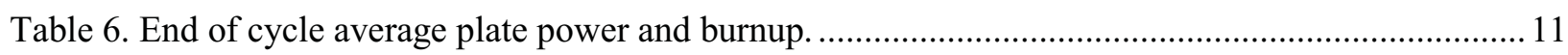

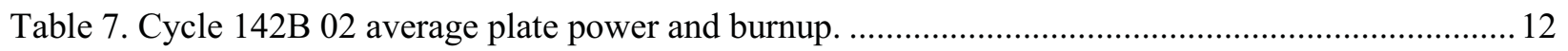

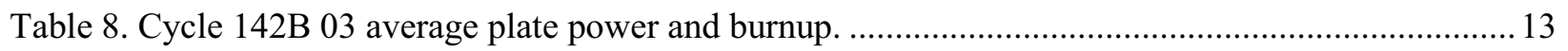

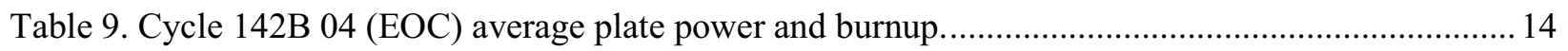

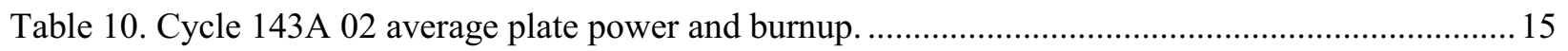

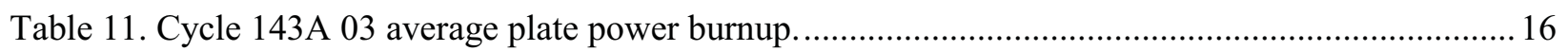

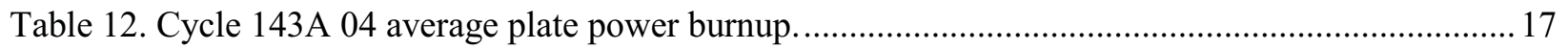

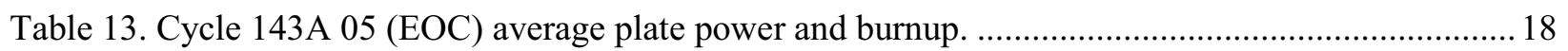

Table 14. Loss coefficients for the RERTR Irradiation Test Vehicle components. ${ }^{7}$................................ 22

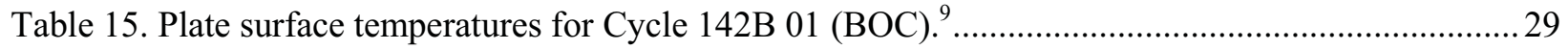

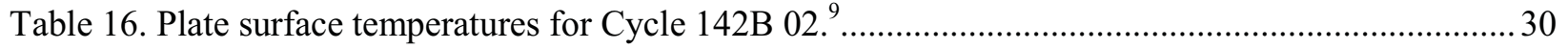

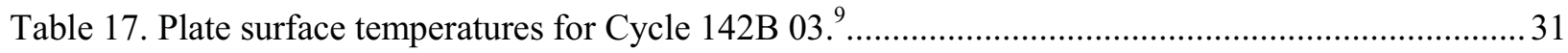

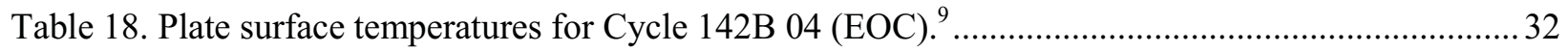

Table 19. Plate surface temperature for Cycle 143A 01 (BOC) ${ }^{9}$............................................................. 33

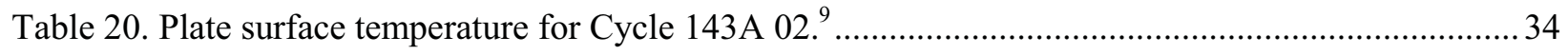

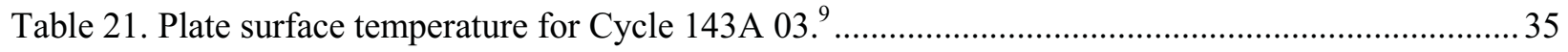

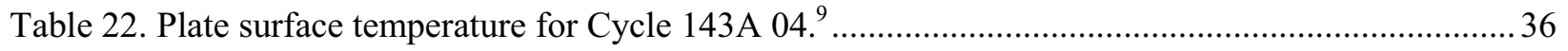






\section{ACRONYMS}

$\begin{array}{ll}\text { Al } & \text { aluminum } \\ \text { ATR } & \text { Advanced Test Reactor } \\ \text { BOC } & \text { beginning of cycle } \\ \text { EFPD } & \text { effective full power days } \\ \text { EOC } & \text { end of cycle } \\ \text { FB } & \text { friction bonding } \\ \text { HIP } & \text { hot isostatic pressing } \\ \text { L2AR } & \text { local-to-average ration } \\ \text { LEU } & \text { low enriched uranium } \\ \text { MCNP } & \text { Monte Carlo N-Particle } \\ \text { Mo } & \text { molybdenum } \\ \text { Nb } & \text { niobium } \\ \text { RERTR } & \text { Reduced Enrichment Research and Test Reactor } \\ \text { Si } & \text { silicon } \\ \text { U } & \text { uranium } \\ \text { U-Mo } & \text { uranium-molybdenum } \\ \text { Zr } & \text { zirconium }\end{array}$




\section{RERTR-10 Irradiation Summary Report \\ 1. EXPERIMENT GOALS}

The Reduced Enrichment Research and Test Reactor (RERTR)-10A/B test assembly holds 4 capsules, designated as A, B, C and D, with A at the top of the assembly and D at the bottom. Each capsule has 2 levels, with 4 plate positions per level, for a total of 8 plate positions per capsule and 32 plate positions per assembly. Within each capsule the 8 plate positions are azimuthally designated as 1 through 4 in the upper level and 5 through 8 in the lower level. The loading diagram for the RERTR-10A/B Experiment Matrix is shown in Table 1.

The RERTR-10 experiment was designed to further test the effectiveness of modified fuel/clad interfaces in monolithic fuel plates. The experiment was conducted in two campaigns: RERTR-10A and RERTR-10B. The fuel plates tested in RERTR-10A were all fabricated by Hot Isostatic Pressing (HIP) and were designed to evaluate the effect of various Si levels in the interlayer and the thickness of the $\mathrm{Zr}$ interlayer (0.001") using 0.010" and 0.020" nominal foil thicknesses. The fuel plates in RERTR-10B were fabricated by Friction Bonding (FB) with two different thickness Si layers and $\mathrm{Nb}$ and $\mathrm{Zr}$ diffusion barriers. ${ }^{1}$ 
Table 1. RERTR-10A/B experiment matrix loading diagram.1RERTR-10A/B Experiment Matrix

\begin{tabular}{|c|c|c|c|c|}
\hline Capsule & Column 1 & Column 2 & Column 3 & Column 4 \\
\hline & A1 & $\mathrm{A} 2$ & $\mathrm{~A} 3$ & A4 \\
\hline A-Top & $\begin{array}{c}\text { Zr Co-Roll } \\
\text { HIP } \\
\text { 0.001” Thick Layer } \\
\text { L1P30Z }\end{array}$ & $\begin{array}{c}\text { Al-12 Si alloy } \\
\text { HIP } \\
0.001 \text { ” Thick Layer } \\
\text { L1P256 }\end{array}$ & $\begin{array}{c}\text { Zr Co-Roll } \\
\text { HIP } \\
\text { Thick Foil } \\
\text { Constrained Plate } \\
\text { L2P15Z }\end{array}$ & $\begin{array}{c}\text { Al-2 Si alloy } \\
\text { HIP } \\
0.001 ” \text { Thick Layer } \\
\text { L1P135 }\end{array}$ \\
\hline & A5 & A6 & A7 & A8 \\
\hline A-Bottom & $\begin{array}{c}\text { Al-3.5 } \mathrm{Si} / \mathrm{Al}+5 \mathrm{Si} \\
\text { HIP } \\
\text { 0.001” Thick Layer } \\
\text { L1P234 }\end{array}$ & $\begin{array}{c}\mathrm{Al}+1.5 \mathrm{Si} / \mathrm{Al}+2 \mathrm{Si} \\
\text { HIP } \\
0.001 ” \text { Thick Layer } \\
\text { L1P213 }\end{array}$ & $\begin{array}{c}\mathrm{Al}+0 \mathrm{Si} / \mathrm{Al}+1 \mathrm{Si} \\
\text { HIP } \\
0.001 ” \text { Thick Layer } \\
\text { L1P192 }\end{array}$ & $\begin{array}{c}\text { thin } \mathrm{Si} \text { /thick Si } \\
\text { HIP } \\
\text { L1P171 }\end{array}$ \\
\hline & B1 & B2 & B3 & B4 \\
\hline B-Top & $\begin{array}{r}\text { Blank } \\
\text { DUMMY } \\
\end{array}$ & $\begin{array}{c}\text { Thin Si/thick Si } \\
\text { FB } \\
\text { Heat Treat } \\
\text { L1F401 } \\
\end{array}$ & $\begin{array}{c}\text { Thin Si/thick Si } \\
\text { FB } \\
\text { No Heat Treat } \\
\text { L1F417 } \\
\end{array}$ & DUMMY \\
\hline & B5 & B6 & B7 & B8 \\
\hline B-Bottom & $\begin{array}{c}\text { Blank } \\
\text { DUMMY } \\
\end{array}$ & $\begin{array}{l}\text { Zr Co-Roll } \\
\text { FB } \\
\text { Thick Foil } \\
\text { L2F46Z } \\
\end{array}$ & $\begin{array}{c}\text { Nb Co-Roll } \\
\text { FB } \\
\text { L1F44N } \\
\end{array}$ & DUMMY \\
\hline & $\mathrm{C} 1$ & $\mathrm{C} 2$ & $\mathrm{C} 3$ & $\mathrm{C} 4$ \\
\hline C-Top & $\begin{array}{c}\text { Zr Co-Roll } \\
\text { HIP } \\
\text { 0.001” Thick Layer } \\
\text { L1P12Z }\end{array}$ & $\begin{array}{c}\text { Al-12 Si alloy } \\
\text { HIP } \\
0.001 \text { ” Thick Layer } \\
\text { L1P266 }\end{array}$ & $\begin{array}{c}\text { Zr Co-Roll } \\
\text { HIP } \\
\text { Thick Foil } \\
\text { Constrained Plate } \\
\text { L2P16Z }\end{array}$ & $\begin{array}{c}\text { Al-2 Si alloy } \\
\text { HIP } \\
0.001 " \text { Thick Layer } \\
\text { L1P145 }\end{array}$ \\
\hline & $\mathrm{C} 5$ & C6 & $\mathrm{C} 7$ & $\mathrm{C} 8$ \\
\hline C-Bottom & $\begin{array}{c}\text { Al-3.5 Si/Al+5 Si } \\
\text { HIP } \\
\text { 0.001” Thick Layer } \\
\text { L1P244 } \\
\end{array}$ & $\begin{array}{c}\mathrm{Al}+1.5 \mathrm{Si} / \mathrm{Al}+2 \mathrm{Si} \\
\mathrm{HIP} \\
0.001 ” \text { Thick Layer } \\
\mathbf{L 1 P 2 2 3} \\
\end{array}$ & $\begin{array}{c}\mathrm{Al}+0 \mathrm{Si} / \mathrm{Al}+1 \mathrm{Si} \\
\text { HIP } \\
0.001 ” \text { Thick Layer } \\
\text { L1P202 }\end{array}$ & $\begin{array}{l}\text { thin } \mathrm{Si} \text { /thick Si } \\
\text { HIP } \\
\text { L1P181 }\end{array}$ \\
\hline & D1 & D2 & D3 & D4 \\
\hline D-Top & $\begin{array}{c}\text { Blank } \\
\text { DUMMY }\end{array}$ & $\begin{array}{c}\text { Zr Co-Roll } \\
\text { FB } \\
\text { L1F381 } \\
\end{array}$ & $\begin{array}{c}\text { Nb Co-Roll } \\
\text { FB } \\
\text { L1F427 } \\
\end{array}$ & $\begin{array}{c}\text { Blank } \\
\text { DUMMY }\end{array}$ \\
\hline & D5 & D6 & D7 & D8 \\
\hline D-Bottom & $\begin{array}{c}\text { Blank } \\
\text { DUMMY }\end{array}$ & $\begin{array}{l}\text { Zr Co-Roll } \\
\text { FB } \\
\text { Thick Foil } \\
\text { L2F45Z }\end{array}$ & $\begin{array}{c}\text { Zr Co-Roll } \\
\text { FB } \\
\text { L2F47Z }\end{array}$ & DUMMY \\
\hline
\end{tabular}




\section{CONSTITUENT MASSES AND DENSITIES}

Table 2. RERTR-10A/B constituent masses and densities. ${ }^{2,3}$

\begin{tabular}{|c|c|c|c|c|c|c|c|}
\hline \multirow[b]{2}{*}{$\begin{array}{c}\text { Fuel } \\
\text { Plate } \\
\text { ID } \\
\end{array}$} & \multirow[b]{2}{*}{$\begin{array}{c}\text { Fuel Plate } \\
\#\end{array}$} & \multicolumn{3}{|c|}{ Fuel Constituent Masses } & \multicolumn{3}{|c|}{ Constituent Densities } \\
\hline & & $\begin{array}{c}\text { Total-U } \\
(\mathrm{g})\end{array}$ & $\begin{array}{c}\mathrm{U}-235 \\
(\mathrm{~g})\end{array}$ & $\begin{array}{l}\text { Mo } \\
(\mathrm{g})\end{array}$ & $\begin{array}{c}\text { Total U } \\
\text { Density } \\
(\mathrm{g} / \mathrm{cc})\end{array}$ & $\begin{array}{c}\text { U-235 } \\
\text { Density } \\
(\mathrm{g} / \mathrm{cc})\end{array}$ & $\begin{array}{c}\text { Mo } \\
(\mathrm{g} / \mathrm{cc})\end{array}$ \\
\hline A1 & L1P30Z & 5.968 & 3.998 & 0.670 & 13.023 & 8.724 & 1.462 \\
\hline $\mathrm{A} 2$ & L1P256 & 6.080 & 4.073 & 0.682 & 15.253 & 10.218 & 1.711 \\
\hline A3 & L2P15Z & 11.522 & 3.845 & 1.298 & 14.258 & 4.758 & 1.606 \\
\hline A4 & L1P135 & 6.234 & 4.177 & 0.759 & 15.279 & 10.238 & 1.860 \\
\hline A5 & L1P234 & 6.669 & 4.468 & 0.747 & 15.144 & 10.146 & 1.696 \\
\hline A6 & L1P213 & 6.261 & 4.195 & 0.698 & 15.268 & 10.230 & 1.702 \\
\hline A7 & L1P192 & 6.553 & 4.390 & 0.730 & 15.522 & 10.398 & 1.729 \\
\hline A8 & L1P171 & 6.230 & 4.174 & 0.696 & 15.285 & 10.241 & 1.708 \\
\hline $\mathrm{B} 1$ & Blank & -- & -- & -- & -- & -- & -- \\
\hline $\mathrm{B} 2$ & L1F401 & 5.624 & 3.768 & 0.630 & 14.578 & 9.767 & 1.633 \\
\hline B3 & L1F417 & 5.730 & 3.839 & 0.648 & 14.326 & 9.598 & 1.620 \\
\hline B4 & Blank & -- & -- & -- & -- & -- & -- \\
\hline B5 & Blank & -- & -- & -- & -- & -- & -- \\
\hline B6 & L2F46Z & 10.365 & 3.431 & 1.160 & 13.154 & 4.354 & 1.472 \\
\hline B7 & L1F44N & 5.419 & 3.631 & 0.605 & 12.449 & 8.341 & 1.390 \\
\hline B8 & Blank & -- & -- & -- & -- & -- & -- \\
\hline $\mathrm{C} 1$ & L1P12Z & 6.009 & 4.026 & 0.470 & 12.492 & 8.370 & 0.977 \\
\hline $\mathrm{C} 2$ & L1P266 & 6.164 & 4.130 & 0.691 & 14.832 & 9.938 & 1.663 \\
\hline $\mathrm{C} 3$ & L2P16Z & 11.354 & 3.789 & 1.278 & 13.676 & 4.564 & 1.539 \\
\hline $\mathrm{C} 4$ & L1P145 & 6.265 & 4.197 & 0.703 & 15.041 & 10.076 & 1.688 \\
\hline $\mathrm{C} 5$ & L1P244 & 6.293 & 4.217 & 0.703 & 15.182 & 10.173 & 1.696 \\
\hline C6 & L1P223 & 6.352 & 4.256 & 0.712 & 15.335 & 10.275 & 1.719 \\
\hline $\mathrm{C} 7$ & L1P202 & 6.406 & 4.292 & 0.718 & 15.585 & 9.772 & 1.635 \\
\hline $\mathrm{C} 8$ & L1P181 & 6.202 & 4.155 & 0.692 & 15.067 & 10.094 & 1.681 \\
\hline D1 & Blank & -- & -- & -- & -- & -- & -- \\
\hline $\mathrm{D} 2$ & L1F381 & 5.624 & 3.768 & 0.630 & 14.997 & 10.048 & 1.680 \\
\hline D3 & L1F427 & 5.707 & 3.824 & 0.642 & 14.983 & 10.039 & 1.685 \\
\hline $\mathrm{D} 4$ & Blank & -- & -- & -- & -- & -- & -- \\
\hline D5 & Blank & -- & -- & -- & -- & -- & -- \\
\hline D6 & L2F45Z & 10.510 & 3.507 & 1.189 & 19.716 & 6.579 & 2.231 \\
\hline D7 & L2F47Z & 10.651 & 3.554 & 1.205 & 13.603 & 4.539 & 1.539 \\
\hline D8 & Blank & -- & -- & -- & -- & -- & -- \\
\hline
\end{tabular}




\section{EXPERIMENT HARDWARE}

The experiment hardware configuration is identical to that used in the RERTR-7A, $-7 \mathrm{~B},-8,-9 \mathrm{~A}$, and -9B experiments. A list of irradiation hardware drawings used for analysis is given in Table 3.

Table 3. RERTR-10 Irradiation Hardware Drawing List. ${ }^{4}$

\begin{tabular}{cl}
$\begin{array}{c}\text { Drawing } \\
\text { Number }\end{array}$ & \multicolumn{1}{c}{ Drawing Title } \\
\hline DWG-630223 & RERTR ATR Large B-Position Irradiation Experiment Assembly \\
DWG-630233 & ATR Large B-Position Basket \\
DWG-630231 & ATR Top Spacer Assembly \\
DWG-630225 & ATR Upper Spacer Assembly \\
DWG-630229 & ATR Bottom Spacer Assembly \\
DWG-630227 & ATR Large B-Position Fuel Capsule Assembly \\
DWG-630237 & Fuel Capsule \\
DWG-630239 & Capsule Cap \\
DWG-630244 & RERTR Mini-Plate \\
DWG-630245 & Fuel Plate, 0.020 Monolithic \\
\hline
\end{tabular}

The RERTR miniplate irradiation assembly ${ }^{4}$, (see Figure 1) shows the main components of the test assembly, which include the bottom spacer, upper and top spacers, experiment capsules and basket. The bottom spacer elevates the experiment capsules to the correct location in the core. The upper and top spacers allow the operators to assure that the experiment is seated fully into the basket. All spacers are similar to the capsule design except the spacers do not have the grooves for the plates. The capsules hold the fuel plates; a capsule cap is welded onto the top of the capsule to keep the plates from sliding out during handling and irradiation. The fuel plate drawings for monolithic and thick monolithic plates (DWG-630244 and DWG-630245, respectively) and RERTR miniplate capsule assembly ${ }^{4}$ are shown in Figure 2, Figure 3 and Figure 4, respectively. Each capsule has a notch at the top and a groove at the bottom which allow the capsules to stack and align properly into the core. The basket holds the test assembly in the reactor during irradiation, the notches on the outer wall allow for bypass coolant flow to cool the outer wall. The basket has two guide bars on the inside wall to guide the assembly into the baskets. 


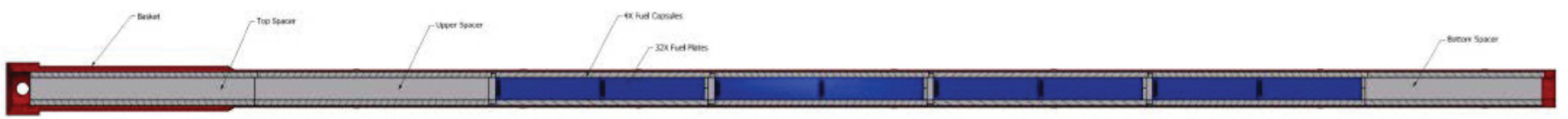

Figure 1 RERTR miniplate irradiation Assembly. ${ }^{4}$ 


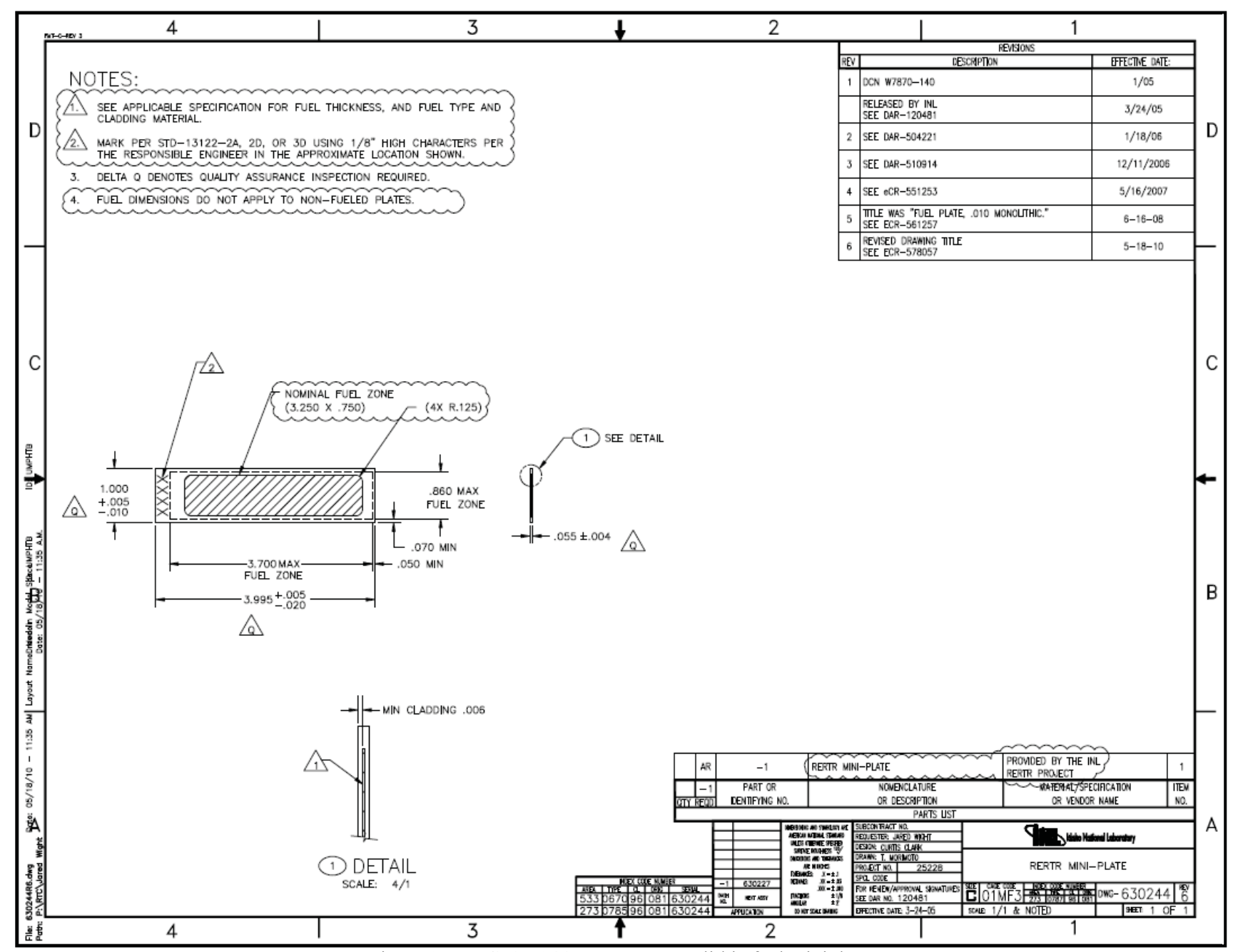

Figure 2. DWG-630244: RERTR monolithic fuel miniplate. 


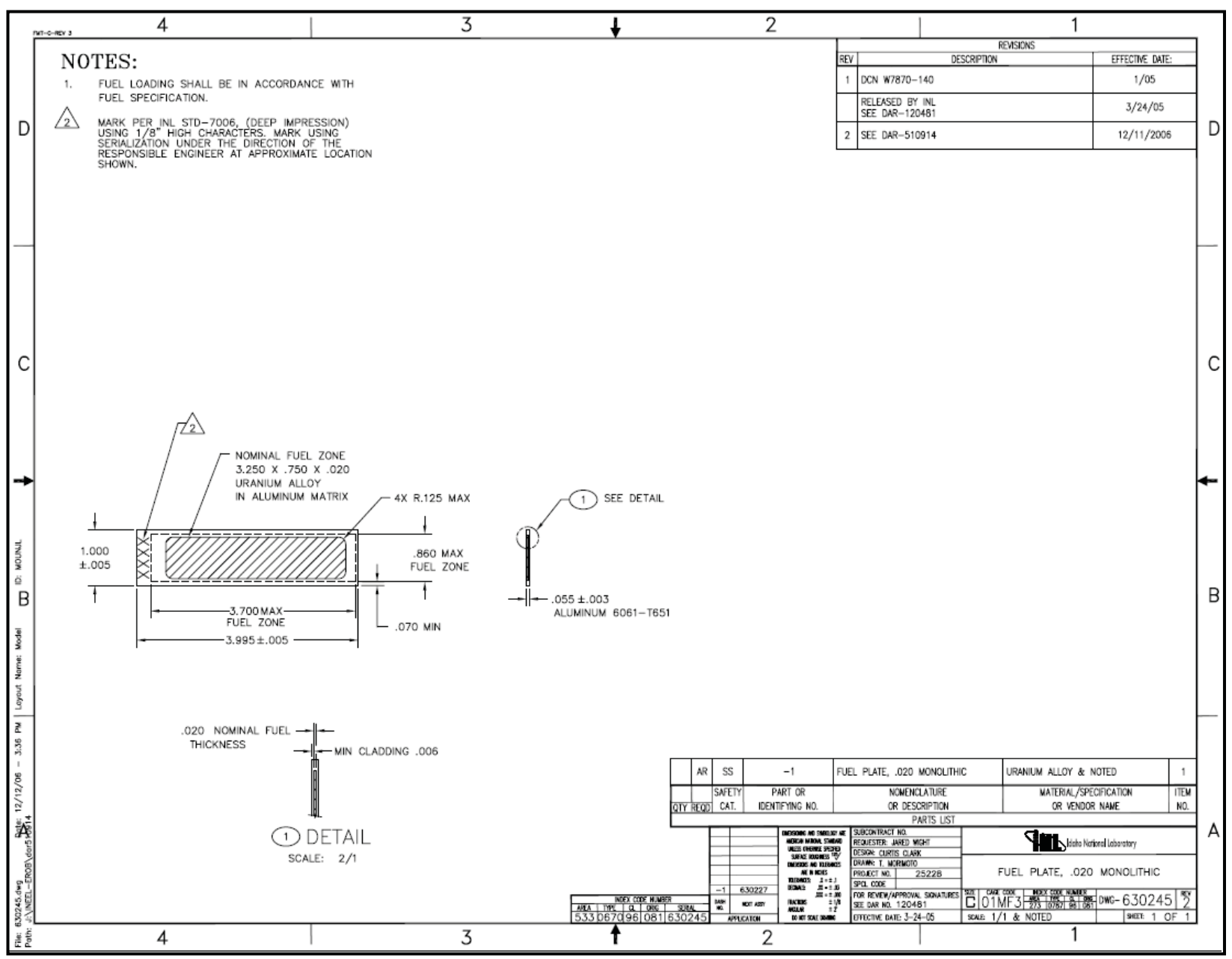

Figure 3. DWG-630245: RERTR thick (0.020 in) monolithic fuel miniplate. 


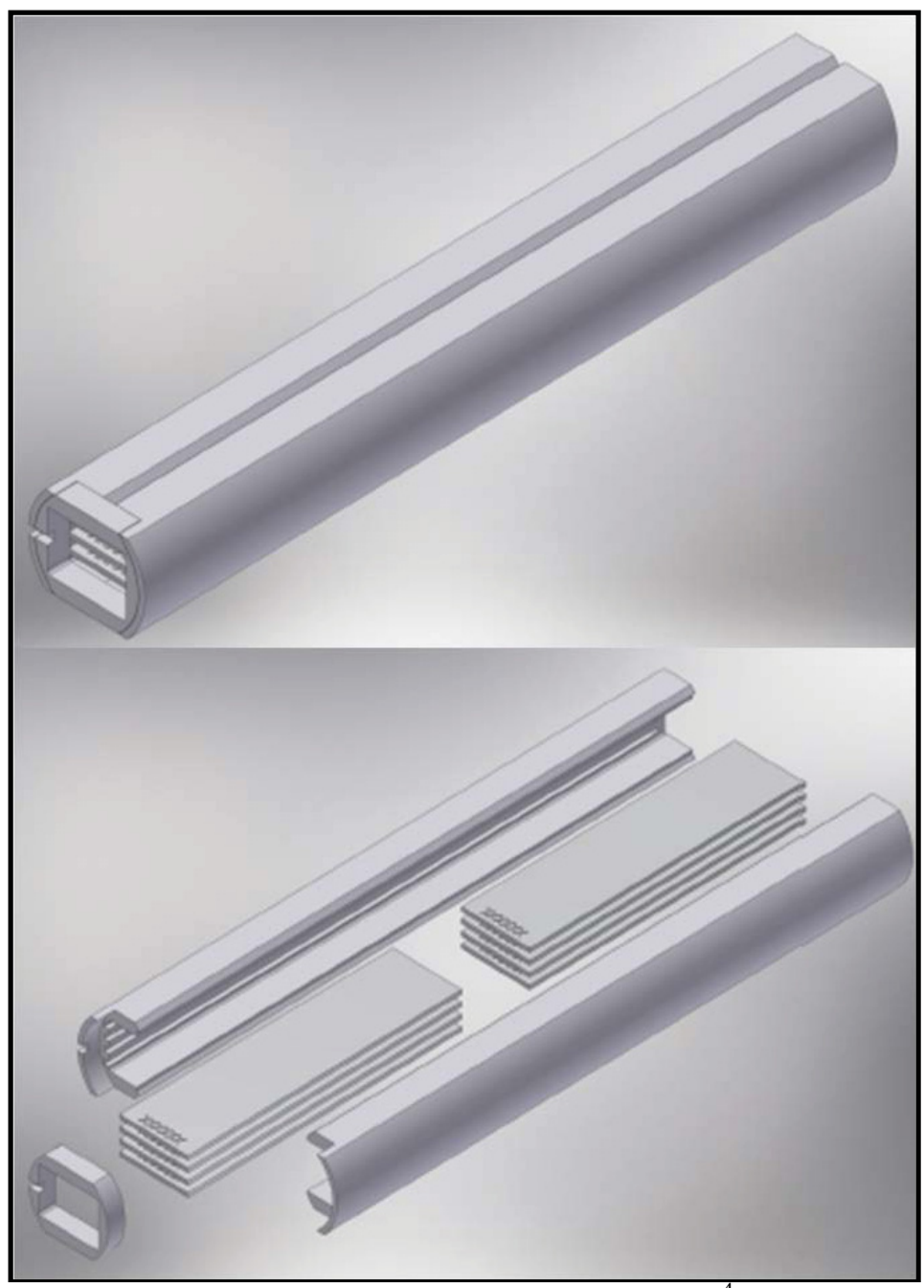

Figure 4. RERTR capsule assembly. ${ }^{4}$ 


\section{IRRADIATION HISTORY}

The RERTR-10A test assembly was irradiated in cycle 142B in the large-B position B-9 and Cycle 143A in the large-B position B-11. The RERTR-10B test assembly was irradiated in Cycle 143A in the large-B position B-11. The RERTR-10A/B test assembly is the combined 10A and 10B test assemblies. The power of position B-9 is represented by the north lobe power which is the average of the $\mathrm{NW}, \mathrm{C}$ and $\mathrm{NE}$ lobe powers, $\mathrm{N}=(\mathrm{NW}+\mathrm{C}+\mathrm{NE}) / 3$. The power of position $\mathrm{B}-11$ is represented by the south lobe power which is the average of the $\mathrm{SW}, \mathrm{C}$ and $\mathrm{SE}$ lobe powers, $\mathrm{S}=(\mathrm{SW}+\mathrm{C}+\mathrm{SE}) / 3$. Cycle 142B ran for 52 EFPDs at 25.3 MW and cycle 143A ran for a total of 48.9 EFPDs at 24.4 MW. RERTR-10A failed (Plate L1P145) during cycle 143A and was removed after 26 EFPDs, RERTR-10B continued irradiation for an additional 22.9 days.

There were two Mid-Cycle SCRAMs during Cycle 142B with a duration of 3 days and 2 days, from dates 07/06/08 - 07/09/08 and 08/05/08 - 08/07/08 respectively. There were also two Mid-Cycle SRAMs during Cycle 143A with duration of 20 days and 5 days, from the dates 10/16/08 - 11/05/08 and 12/21/08 $-12 / 26 / 08$ respectively. This information is tabulated in Table 4.

Table 4. Irradiation history for RERTR-10.

\begin{tabular}{|c|c|c|c|c|c|c|c|c|}
\hline $\begin{array}{c}\text { ATR } \\
\text { CYCLE }\end{array}$ & $\begin{array}{c}\text { RERTR-10A/B } \\
\text { Test ID }\end{array}$ & $\begin{array}{l}\text { RERTR-10 } \\
\text { Capsules } \\
\text { Irradiated }\end{array}$ & $\begin{array}{c}\text { Dates } \\
\text { Irradiated }\end{array}$ & $\begin{array}{l}\text { Cycle } \\
\text { EFPDs }\end{array}$ & $\begin{array}{c}\text { Mid- } \\
\text { Cycle } \\
\text { Scram } \\
\text { Decay } \\
\text { Days }\end{array}$ & $\begin{array}{l}\text { Post- } \\
\text { Cycle } \\
\text { Decay } \\
\text { Days }\end{array}$ & $\begin{array}{l}\text { North } \\
\text { Lobe } \\
\text { Source } \\
\text { Power } \\
\text { (MW) }\end{array}$ & $\begin{array}{c}\text { South } \\
\text { Lobe } \\
\text { Source } \\
\text { Power } \\
\text { (MW) }\end{array}$ \\
\hline 142B & RERTR-10A & $\mathrm{A}, \mathrm{C}$ & $\begin{array}{c}07 / 04 / 2008- \\
08 / 30 / 2008\end{array}$ & 52 & 5 & 25 & 22.1 & -- \\
\hline $143 \mathrm{~A}-1$ & RERTR-10A/B & $\mathrm{A}, \mathrm{B}, \mathrm{C}, \mathrm{D}$ & \multirow{2}{*}{$\begin{array}{c}09 / 24 / 2008- \\
12 / 06 / 2008\end{array}$} & 26.0 & \multirow{2}{*}{25} & -- & -- & 25.4 \\
\hline $143 \mathrm{~A}-2$ & RERTR-10B & $\mathrm{B}, \mathrm{D}$ & & 22.9 & & 17 & -- & 25.4 \\
\hline
\end{tabular}




\section{AS-RUN NUCLEAR ANALYSIS}

\subsection{Neutronics}

The as-run calculations were performed using the irradiation history in Table 4 and the Monte Carlo N-Particle (MCNP) code. The calculated as-run fission heat rates, fission densities, and as-run U-235 burnup results for the fueled miniplates reported have an uncertainty band $(1 \sigma)$ of $2.5 \% .{ }^{5}$ The time intervals used to calculate the average plate power and burnup is shown in Table 5 . The end of cycle average plate power and burnup for cycles 142B and 143A are shown in Table 6 . The average plate power and burnup for the time intervals for each cycle are shown in Table 7 through Table 13. The plots of the power and fission density as a function of the ATR Cycle time interval are in Appendix A.

Table 5. Cycle breakdown.

\begin{tabular}{ccc}
\hline $\begin{array}{c}\text { Time } \\
\text { Interval }\end{array}$ & $\begin{array}{c}142 \mathrm{~B} \\
\text { (days) }\end{array}$ & $\begin{array}{c}143 \mathrm{~A} \\
\text { (days) }\end{array}$ \\
\hline 01 & $1.00 \mathrm{E}-04$ & $1.00 \mathrm{E}-04$ \\
02 & 16 & 15 \\
03 & 13.7 & 11 \\
04 & 22.3 & 11 \\
05 & $1.00 \mathrm{E}-04$ & 11.9 \\
06 & -- & $1.00 \mathrm{E}-04$ \\
EFPDs & 52 & 48.9 \\
\hline
\end{tabular}


Table 6. End of cycle average plate power and burnup.

\begin{tabular}{|c|c|c|c|c|c|c|c|c|c|c|c|c|c|c|c|c|}
\hline \multirow[b]{2}{*}{ Position ID } & \multirow[b]{2}{*}{ Plate ID } & \multicolumn{5}{|c|}{ 142B 52.0 EFPD (EOC) } & \multicolumn{5}{|c|}{ 143A 26.0 EFPD (EOC for RERTR-10A) } & \multicolumn{5}{|c|}{ 143A 48.9 EFPD (EOC for RERTR-10B) } \\
\hline & & $\begin{array}{l}\text { Fission } \\
\text { Heat } \\
\text { Rate } \\
(\mathrm{W} / \mathrm{g})\end{array}$ & $\begin{array}{l}\text { Fission } \\
\text { Power } \\
\text { Density } \\
\left(\mathrm{w} / \mathrm{cm}^{3}\right)\end{array}$ & $\begin{array}{c}\text { Heat Flux } \\
\left(\mathrm{w} / \mathrm{cm}^{2}\right)\end{array}$ & \begin{tabular}{|c|} 
U-235 \\
Burnup u- \\
$235 / \mathrm{u}-$ \\
$235_{\text {initial }}$ \\
(\%)
\end{tabular} & $\begin{array}{c}\text { Fission } \\
\text { Density } \\
\text { (f/cc) }\end{array}$ & $\begin{array}{c}\text { Fission } \\
\text { Heat } \\
\text { Rate } \\
(\mathrm{W} / \mathrm{g})\end{array}$ & $\begin{array}{l}\text { Fission } \\
\text { Power } \\
\text { Density } \\
\left(\mathrm{w} / \mathrm{cm}^{3}\right)\end{array}$ & $\begin{array}{l}\text { Heat Flux } \\
\left(\mathrm{w} / \mathrm{cm}^{2}\right)\end{array}$ & \begin{tabular}{|c|} 
U-235 \\
Burnup u- \\
$235 / \mathrm{u}-$ \\
$235_{\text {initial }}$ \\
$(\%)$
\end{tabular} & $\begin{array}{c}\text { Fission } \\
\text { Density } \\
\text { (f/cc) }\end{array}$ & $\begin{array}{c}\text { Fission } \\
\text { Heat } \\
\text { Rate } \\
(\mathrm{W} / \mathrm{g})\end{array}$ & $\begin{array}{l}\text { Fission } \\
\text { Power } \\
\text { Density } \\
\left(\mathrm{w} / \mathrm{cm}^{3}\right)\end{array}$ & \begin{tabular}{|c} 
Heat Flux \\
$\left(\mathrm{w} / \mathrm{cm}^{2}\right)$
\end{tabular} & \begin{tabular}{|c|} 
U-235 \\
Burnup u- \\
$235 / \mathrm{u}-$ \\
$235_{\text {initial }}$ \\
$(\%)$
\end{tabular} & $\begin{array}{l}\text { Fission } \\
\text { Density } \\
\text { (f/cc) }\end{array}$ \\
\hline A1 & L1P30Z & 821.40 & 11401.91 & 166.47 & $8.99 \%$ & $1.70 \mathrm{E}+21$ & 1099.96 & 14806.39 & 216.17 & $15.17 \%$ & $2.88 \mathrm{E}+21$ & -- & -- & -- & -- & -- \\
\hline A2 & L1P256 & 684.15 & 11207.49 & 145.14 & $7.45 \%$ & $1.64 \mathrm{E}+21$ & 928.89 & 14828.31 & 192.03 & $12.72 \%$ & $2.81 \mathrm{E}+21$ & -- & -- & -- & -- & -- \\
\hline $\mathrm{A} 3$ & L2P15Z & 340.31 & 5304.89 & 144.82 & $7.55 \%$ & $7.78 \mathrm{E}+20$ & 463.15 & 7126.35 & 194.55 & $12.88 \%$ & $1.34 \mathrm{E}+21$ & -- & -- & -- & -- & -- \\
\hline A4 & L1P135 & 820.48 & 13486.86 & 176.68 & $8.88 \%$ & $1.98 \mathrm{E}+21$ & 1136.69 & 18104.54 & 237.17 & $15.33 \%$ & $3.42 \mathrm{E}+21$ & -- & -- & -- & -- & -- \\
\hline A5 & L1P234 & 949.02 & 15214.06 & 210.71 & $10.35 \%$ & $2.29 \mathrm{E}+21$ & 1331.66 & 20568.00 & 284.87 & $17.77 \%$ & $3.94 \mathrm{E}+21$ & -- & -- & -- & -- & -- \\
\hline A6 & L1P213 & 788.96 & 12854.57 & 168.39 & $8.66 \%$ & $1.91 \mathrm{E}+21$ & 1129.86 & 17842.64 & 233.74 & $14.96 \%$ & $3.31 \mathrm{E}+21$ & - & -- & -- & -- & -- \\
\hline A7 & L1P192 & 797.99 & 13213.14 & 177.72 & $8.71 \%$ & $1.96 \mathrm{E}+21$ & 1136.23 & 18229.56 & 245.19 & $15.05 \%$ & $3.39 \mathrm{E}+21$ & -- & -- & -- & -- & -- \\
\hline A8 & L1P171 & 999.24 & 16128.87 & 208.87 & $10.79 \%$ & $2.41 \mathrm{E}+21$ & 1396.15 & 21670.62 & 280.63 & $18.52 \%$ & $4.15 \mathrm{E}+21$ & -- & -- & -- & -- & -- \\
\hline B1 & Blank & -- & -- & -- & -- & -- & -- & -- & -- & -- & -- & -- & -- & -- & -- & -- \\
\hline B2 & L1F401 & -- & -- & -- & -- & -- & 1892.10 & 29075.16 & 357.62 & $11.15 \%$ & $2.42 \mathrm{E}+21$ & \begin{tabular}{|l|}
2096.89 \\
\end{tabular} & \begin{tabular}{|l|}
30619.84 \\
\end{tabular} & 376.62 & $21.14 \%$ & $4.58 \mathrm{E}+21$ \\
\hline B3 & L1F417 & -- & -- & -- & -- & -- & 1898.52 & 28688.84 & 364.35 & $11.22 \%$ & $2.39 \mathrm{E}+21$ & \begin{tabular}{|l|}
2184.18 \\
\end{tabular} & \begin{tabular}{|l|}
31295.20 \\
\end{tabular} & 397.45 & $21.63 \%$ & $4.61 \mathrm{E}+21$ \\
\hline B4 & Blank & -- & -- & -- & -- & -- & -- & -- & -- & -- & -- & -- & -- & -- & -- & -- \\
\hline B5 & Blank & -- & -- & -- & -- & -- & -- & -- & -- & -- & -- & -- & -- & -- & -- & -- \\
\hline B6 & L2F46Z & -- & -- & -- & -- & -- & 1004.61 & 14269.85 & 360.31 & $12.34 \%$ & $1.20 \mathrm{E}+21$ & \begin{tabular}{|l|}
1072.37 \\
\end{tabular} & \begin{tabular}{|l|}
14835.00 \\
\end{tabular} & 374.58 & $23.09 \%$ & \begin{tabular}{|l|}
$2.25 \mathrm{E}+21$ \\
\end{tabular} \\
\hline B7 & L1F44N & -- & -- & -- & -- & -- & 2066.83 & 26963.40 & 373.44 & $12.26 \%$ & $2.28 \mathrm{E}+21$ & \begin{tabular}{|l|}
2341.33 \\
\end{tabular} & \begin{tabular}{|l|}
28846.91 \\
\end{tabular} & 399.53 & $23.30 \%$ & $4.34 \mathrm{E}+21$ \\
\hline B8 & Blank & -- & -- & -- & -- & -- & -- & -- & -- & -- & -- & -- & -- & -- & - & -- \\
\hline C1 & L1P12Z & 1175.14 & 14868.69 & 230.46 & $12.63 \%$ & $2.32 \mathrm{E}+21$ & 1729.95 & 20833.42 & 322.92 & $21.96 \%$ & $4.04 \mathrm{E}+21$ & - & -- & -- & -- & -- \\
\hline $\mathrm{C} 2$ & L1P266 & 956.51 & 14999.26 & 194.24 & $10.64 \%$ & $2.30 \mathrm{E}+21$ & 1420.50 & 21400.87 & 277.14 & $18.62 \%$ & $4.03 E+21$ & - & -- & -- & - & - \\
\hline C3 & L2P16Z & 484.92 & 7190.58 & 192.71 & $11.03 \%$ & $1.09 \mathrm{E}+21$ & 698.60 & 10153.41 & 272.11 & $19.14 \%$ & $1.91 \mathrm{E}+21$ & - & -- & -- & -- & - \\
\hline C4 & L1P145 & 1152.20 & 18142.24 & 242.20 & $12.66 \%$ & $2.79 \mathrm{E}+21$ & 1714.03 & 25701.43 & 343.11 & $22.15 \%$ & $4.91 \mathrm{E}+21$ & - & -- & -- & -- & - \\
\hline C5 & L1P244 & 1092.63 & 17404.31 & 229.74 & $12.09 \%$ & $2.69 \mathrm{E}+21$ & 1579.83 & 24057.65 & 317.56 & $20.84 \%$ & $4.67 \mathrm{E}+21$ & - & - & -- & - & -- \\
\hline C6 & L1P223 & 894.78 & 14554.25 & 192.12 & $9.99 \%$ & $2.23 E+21$ & 1325.31 & 20766.44 & 274.12 & $17.44 \%$ & $3.90 \mathrm{E}+21$ & - & - & $\overline{--}$ & - & - \\
\hline C7 & L1P202 & 914.03 & 14134.78 & 196.47 & $10.06 \%$ & $2.13 \mathrm{E}+21$ & 1346.40 & 20049.97 & 278.69 & $17.61 \%$ & $3.75 \mathrm{E}+21$ & -- & -- & -- & -- & -- \\
\hline C8 & L1P181 & 1139.17 & $\begin{array}{l}17977.59 \\
\end{array}$ & 237.30 & $12.37 \%$ & $2.73 E+21$ & 1646.89 & 24802.49 & 327.39 & $21.54 \%$ & $4.78 \mathrm{E}+21$ & - & - & -- & - & -- \\
\hline D1 & Blank & -- & -- & -- & -- & -- & -- & -- & -- & -- & -- & -- & -- & -- & -- & -- \\
\hline D2 & L1F381 & -- & -- & -- & -- & -- & 1719.78 & 27320.47 & 333.31 & $10.21 \%$ & $2.26 \mathrm{E}+21$ & \begin{tabular}{|l|}
1946.23 \\
\end{tabular} & \begin{tabular}{|l|}
29488.28 \\
\end{tabular} & 359.76 & $19.53 \%$ & $4.33 \mathrm{E}+21$ \\
\hline D3 & \begin{tabular}{|l} 
L1F4127 \\
\end{tabular} & -- & -- & - & -- & -- & 1757.60 & 27885.63 & 340.20 & $10.30 \%$ & $2.29 \mathrm{E}+21$ & 2035.30 & \begin{tabular}{|l|}
30729.74 \\
\end{tabular} & 374.90 & $20.06 \%$ & $4.47 \mathrm{E}+21$ \\
\hline D4 & Blank & -- & -- & -- & -- & -- & -- & -- & -- & -- & -- & -- & -- & -- & - & -- \\
\hline D5 & Blank & -- & -- & -- & - & -- & -- & -- & -- & -- & -- & -- & -- & -- & -- & -- \\
\hline D6 & L2F45Z & -- & -- & -- & -- & -- & 644.52 & 13888.14 & 336.79 & $7.83 \%$ & $1.12 \mathrm{E}+21$ & 724.63 & \begin{tabular}{|l|}
15339.81 \\
\end{tabular} & 371.99 & $15.12 \%$ & \begin{tabular}{|l|}
$2.19 \mathrm{E}+21$ \\
\end{tabular} \\
\hline D7 & L2F47Z & -- & -- & -- & -- & -- & 736.74 & 10924.97 & 269.30 & $8.86 \%$ & $8.87 \mathrm{E}+20$ & 846.70 & \begin{tabular}{|l|}
12293.89 \\
\end{tabular} & 303.04 & $17.46 \%$ & $1.75 \mathrm{E}+21$ \\
\hline D8 & Blank & -- & -- & -- & -- & -- & -- & - & -- & -- & -- & -- & -- & -- & -- & -- \\
\hline
\end{tabular}


Table 7. Cycle 142B 02 average plate power and burnup.

\begin{tabular}{|c|c|c|c|c|c|c|c|c|}
\hline \multirow[b]{2}{*}{ Position ID } & \multirow[b]{2}{*}{ Plate ID } & \multicolumn{7}{|c|}{ 142B 16.0 EFPD } \\
\hline & & $\begin{array}{l}\text { Fission } \\
\text { Heat } \\
\text { Rate } \\
(\mathrm{W} / \mathrm{g}) \\
\end{array}$ & $\begin{array}{l}\text { Fission } \\
\text { Power } \\
\text { Density } \\
\left(\mathrm{w} / \mathrm{cm}^{3}\right) \\
\end{array}$ & $\begin{array}{l}\text { Heat } \\
\text { Flux } \\
\left(\mathrm{w} / \mathrm{cm}^{2}\right)\end{array}$ & $\begin{array}{c}\text { U-235 } \\
\text { Burnup u- } \\
235 / \mathrm{u}- \\
235_{\text {initial }}(\%)\end{array}$ & $\begin{array}{l}\text { LEUeq } \\
\text { Burnup } \\
\quad(\%)\end{array}$ & $\begin{array}{c}\text { Fission } \\
\text { Density } \\
\text { (f/cc) }\end{array}$ & $\begin{array}{l}\text { Fission } \\
\text { Rate (f/cc } \\
\mathrm{s})\end{array}$ \\
\hline $\bar{A} 1$ & L1P30Z & 770.84 & 11018.69 & 160.87 & $2.82 \%$ & $9.45 \%$ & $5.40 \mathrm{E}+20$ & $3.91 \mathrm{E}+14$ \\
\hline A2 & L1P256 & 635.02 & 10656.73 & 138.00 & $2.33 \%$ & $7.81 \%$ & $5.14 \mathrm{E}+20$ & $3.72 \mathrm{E}+14$ \\
\hline A3 & L2P15Z & 320.49 & 5056.48 & 138.04 & $2.30 \%$ & $3.84 \%$ & $2.43 E+20$ & $1.76 \mathrm{E}+14$ \\
\hline A4 & L1P135 & 760.17 & 12861.48 & 168.49 & $2.78 \%$ & $9.31 \%$ & $6.22 \mathrm{E}+20$ & $4.50 \mathrm{E}+14$ \\
\hline A5 & L1P234 & 898.01 & 14890.05 & 206.23 & $3.35 \%$ & $11.22 \%$ & $7.34 \mathrm{E}+20$ & $5.31 \mathrm{E}+14$ \\
\hline A6 & L1P213 & 732.86 & 12277.63 & 160.84 & $2.75 \%$ & $9.21 \%$ & $6.15 \mathrm{E}+20$ & $4.45 \mathrm{E}+14$ \\
\hline A7 & L1P192 & 744.97 & 12687.99 & 17.65 & $2.74 \%$ & $9.18 \%$ & $6.21 \mathrm{E}+20$ & $4.49 \mathrm{E}+14$ \\
\hline A8 & L1P171 & 928.78 & 15528.47 & 201.09 & $3.47 \%$ & $11.62 \%$ & $7.72 \mathrm{E}+20$ & $5.58 \mathrm{E}+14$ \\
\hline B1 & Blank & -- & -- & -- & -- & -- & -- & -- \\
\hline B2 & L1F401 & -- & -- & -- & -- & -- & -- & -- \\
\hline B3 & L1F417 & -- & -- & -- & - & -- & -- & -- \\
\hline B4 & Blank & -- & -- & -- & -- & $\overline{--}$ & $\overline{--}$ & -- \\
\hline B5 & Blank & -- & -- & -- & -- & -- & $\overline{--}$ & -- \\
\hline $\mathrm{B} 6$ & L2F46Z & $\overline{--}$ & $\overline{--}$ & $\overline{--}$ & $\overline{--}$ & -- & -- & -- \\
\hline B7 & L1F44N & -- & -- & -- & -- & -- & -- & -- \\
\hline B8 & Blank & $\overline{--}$ & $\overline{--}$ & $\overline{--}$ & $\overline{--}$ & $\overline{--}$ & $\overline{--}$ & $\overline{--}$ \\
\hline C1 & L1P12Z & 1113.38 & 14689.89 & 227.69 & $4.29 \%$ & $14.37 \%$ & $7.80 \mathrm{E}+20$ & $5.64 \mathrm{E}+14$ \\
\hline $\mathrm{C} 2$ & L1P266 & 904.48 & 14675.00 & 190.04 & $3.53 \%$ & $11.83 \%$ & $7.64 \mathrm{E}+20$ & $5.53 \mathrm{E}+14$ \\
\hline $\mathrm{C} 3$ & L2P16Z & 464.35 & 7006.12 & 187.76 & $3.68 \%$ & $6.14 \%$ & $3.57 \mathrm{E}+20$ & $2.58 \mathrm{E}+14$ \\
\hline C4 & L1P145 & 1092.94 & 17923.88 & 239.28 & $4.22 \%$ & $14.14 \%$ & $9.28 \mathrm{E}+20$ & $6.71 \mathrm{E}+14$ \\
\hline C5 & L1P244 & 1040.14 & 17226.34 & 227.39 & $3.99 \%$ & $13.37 \%$ & $8.97 \mathrm{E}+20$ & $6.49 \mathrm{E}+14$ \\
\hline C6 & L1P223 & 850.65 & 14286.10 & 188.58 & $3.27 \%$ & $10.95 \%$ & $7.37 \mathrm{E}+20$ & $5.33 \mathrm{E}+14$ \\
\hline C7 & L1P202 & 842.38 & 13452.64 & 186.99 & $3.35 \%$ & $11.22 \%$ & $7.10 \mathrm{E}+20$ & $5.14 \mathrm{E}+14$ \\
\hline C8 & L1P181 & 1065.58 & 17506.17 & 231.08 & $4.06 \%$ & $13.60 \%$ & $9.01 \mathrm{E}+20$ & $6.52 \mathrm{E}+14$ \\
\hline D1 & Blank & -- & -- & -- & -- & -- & -- & -- \\
\hline D2 & L1F381 & -- & -- & -- & -- & -- & -- & -- \\
\hline D3 & L1F4127 & -- & -- & -- & -- & -- & -- & -- \\
\hline D4 & Blank & -- & -- & -- & -- & -- & -- & -- \\
\hline D5 & Blank & $\overline{--}$ & -- & -- & -- & -- & -- & $\overline{--}$ \\
\hline $\mathrm{D} 6$ & L2F45Z & $\overline{--}$ & $\overline{--}$ & -- & -- & -- & -- & $\overline{--}$ \\
\hline D7 & L2F47Z & $\overline{--}$ & $\overline{--}$ & $\overline{--}$ & $\overline{--}$ & $\overline{--}$ & -- & $\overline{--}$ \\
\hline$\overline{D 8}$ & Blank & -- & -- & -- & -- & -- & -- & -- \\
\hline
\end{tabular}


Table 8. Cycle 142B 03 average plate power and burnup.

\begin{tabular}{|c|c|c|c|c|c|c|c|c|}
\hline \multirow[b]{2}{*}{ Position ID } & \multirow[b]{2}{*}{ Plate ID } & \multicolumn{7}{|c|}{ 142B 29.7 EFPD } \\
\hline & & $\begin{array}{c}\text { Fission } \\
\text { Heat Rate } \\
(\mathrm{W} / \mathrm{g})\end{array}$ & $\begin{array}{l}\text { Fission } \\
\text { Power } \\
\text { Density } \\
\left(\mathrm{w} / \mathrm{cm}^{3}\right)\end{array}$ & $\begin{array}{c}\text { Heat } \\
\text { Flux } \\
\left(\mathrm{w} / \mathrm{cm}^{2}\right)\end{array}$ & $\begin{array}{c}\text { U-235 } \\
\text { Burnup u- } \\
235 / \mathrm{u}-235_{\text {initial }} \\
(\%)\end{array}$ & $\begin{array}{c}\text { LEUeq } \\
\text { Burnup } \\
(\%)\end{array}$ & $\begin{array}{c}\text { Fission } \\
\text { Density } \\
\text { (f/cc) }\end{array}$ & $\begin{array}{l}\text { Fission } \\
\text { Rate } \\
\text { (f/cc-s) }\end{array}$ \\
\hline$\overline{\mathrm{A} 1}$ & L1P30Z & 751.70 & 10625.64 & 155.13 & $5.19 \%$ & $17.39 \%$ & $9.86 \mathrm{E}+20$ & $3.84 \mathrm{E}+14$ \\
\hline A2 & L1P256 & 623.64 & 10369.17 & 134.28 & $4.32 \%$ & $14.47 \%$ & $9.45 E+20$ & $3.68 \mathrm{E}+14$ \\
\hline A3 & L2P15Z & 311.87 & 4897.55 & 133.70 & $4.35 \%$ & $7.26 \%$ & $4.48 \mathrm{E}+20$ & $1.75 E+14$ \\
\hline A4 & L1P135 & 742.40 & 12423.16 & 162.74 & $5.15 \%$ & $17.25 \%$ & $1.14 \mathrm{E}+21$ & $4.44 \mathrm{E}+14$ \\
\hline A5 & L1P234 & 860.20 & 14080.95 & 192.02 & $6.08 \%$ & $20.37 \%$ & $1.34 \mathrm{E}+21$ & $5.22 \mathrm{E}+14$ \\
\hline $\mathrm{A} 6$ & L1P213 & 715.37 & 11858.03 & 155.34 & $5.04 \%$ & $16.88 \%$ & $1.11 \mathrm{E}+21$ & $4.33 \mathrm{E}+14$ \\
\hline A7 & L1P192 & 723.07 & 12182.80 & 163.86 & $5.07 \%$ & $16.98 \%$ & $1.13 \mathrm{E}+21$ & $4.40 \mathrm{E}+14$ \\
\hline A8 & L1P171 & 904.24 & 14918.99 & 193.20 & $6.29 \%$ & $21.07 \%$ & $1.40 E+21$ & $5.46 \mathrm{E}+14$ \\
\hline B1 & Blank & -- & -- & -- & -- & -- & -- & -- \\
\hline B2 & L1F401 & -- & -- & -- & -- & -- & -- & -- \\
\hline B3 & L1F417 & -- & -- & -- & -- & -- & -- & -- \\
\hline B4 & Blank & $\overline{--}$ & $\overline{--}$ & - & $\overline{--}$ & $\overline{--}$ & -- & -- \\
\hline B5 & Blank & $\overline{--}$ & $\overline{--}$ & - & $\overline{--}$ & $\overline{--}$ & -- & -- \\
\hline $\mathrm{B} 6$ & L2F46Z & $\overline{--}$ & $\overline{--}$ & $\overline{--}$ & $\overline{--}$ & $\overline{--}$ & $\overline{--}$ & $\overline{--}$ \\
\hline B7 & L1F44N & -- & - & -- & -- & -- & -- & -- \\
\hline B8 & Blank & $\overline{--}$ & $\overline{--}$ & -- & -- & $\overline{--}$ & -- & $\overline{--}$ \\
\hline C1 & L1P12Z & 1075.32 & 13963.82 & 216.44 & $7.55 \%$ & $25.29 \%$ & $\begin{array}{l}1.37 \mathrm{E}+21 \\
\end{array}$ & $5.34 \mathrm{E}+14$ \\
\hline $\mathrm{C} 2$ & L1P266 & 872.27 & 13970.45 & 180.92 & $6.32 \%$ & $21.17 \%$ & $1.36 \mathrm{E}+21$ & $5.30 \mathrm{E}+14$ \\
\hline $\mathrm{C} 3$ & L2P16Z & 445.61 & 6678.12 & 178.97 & $6.50 \%$ & $10.85 \%$ & $6.41 \mathrm{E}+20$ & $2.50 \mathrm{E}+14$ \\
\hline $\mathrm{C} 4$ & L1P145 & 1040.78 & 16801.73 & 224.30 & $7.55 \%$ & $25.29 \%$ & $1.65 E+21$ & $6.43 E+14$ \\
\hline C5 & L1P244 & 993.96 & 16217.71 & 214.07 & $7.18 \%$ & $24.05 \%$ & $1.59 \mathrm{E}+21$ & $6.20 \mathrm{E}+14$ \\
\hline $\mathrm{C6}$ & L1P223 & 814.88 & 13517.89 & \begin{tabular}{|l|}
178.44 \\
\end{tabular} & $5.89 \%$ & $19.73 \%$ & \begin{tabular}{|l|}
$1.32 \mathrm{E}+21$ \\
\end{tabular} & $5.14 \mathrm{E}+14$ \\
\hline C7 & L1P202 & 826.20 & 13035.00 & 181.19 & $5.95 \%$ & $19.93 \%$ & $1.25 \mathrm{E}+21$ & $4.87 \mathrm{E}+14$ \\
\hline $\mathrm{C} 8$ & L1P181 & 1028.18 & 16635.27 & 219.59 & $7.31 \%$ & $24.49 \%$ & $1.61 \mathrm{E}+21$ & $6.27 \mathrm{E}+14$ \\
\hline D1 & Blank & -- & -- & -- & -- & -- & -- & -- \\
\hline D2 & L1F381 & -- & -- & -- & -- & -- & -- & -- \\
\hline D3 & L1F4127 & -- & -- & -- & -- & -- & -- & -- \\
\hline D4 & Blank & -- & -- & -- & -- & -- & -- & -- \\
\hline D5 & Blank & -- & - & - & -- & - & -- & -- \\
\hline D6 & L2F45Z & -- & - & - & $\overline{--}$ & - & - & -- \\
\hline D7 & L2F47Z & -- & - & -- & -- & -- & -- & -- \\
\hline D8 & Blank & -- & -- & - & - & -- & -- & -- \\
\hline
\end{tabular}


Table 9. Cycle 142B 04 (EOC) average plate power and burnup.

\begin{tabular}{|c|c|c|c|c|c|c|c|c|}
\hline \multirow[b]{2}{*}{$\begin{array}{l}\text { Position } \\
\text { ID }\end{array}$} & \multirow[b]{2}{*}{ Plate ID } & \multicolumn{7}{|c|}{ 142B 52.0 EFPD (EOC) } \\
\hline & & $\begin{array}{c}\text { Fission } \\
\text { Heat Rate } \\
\text { (W/g) }\end{array}$ & $\begin{array}{l}\text { Fission } \\
\text { Power } \\
\text { Density } \\
\text { (w/cm3) }\end{array}$ & $\begin{array}{c}\text { Heat Flux } \\
(\mathrm{w} / \mathrm{cm} 2)\end{array}$ & $\begin{array}{c}\text { U-235 } \\
\text { Burnup u- } \\
\text { 235/u- } \\
\text { 235initial } \\
(\%)\end{array}$ & $\begin{array}{l}\text { LEUeq } \\
\text { Burnup } \\
(\%)\end{array}$ & $\begin{array}{l}\text { Fission } \\
\text { Density } \\
\text { (f/cc) }\end{array}$ & $\begin{array}{l}\text { Fission } \\
\text { Rate } \\
\text { (f/cc-s) }\end{array}$ \\
\hline $\mathrm{A} 1$ & L1P30Z & 821.40 & 11401.91 & 166.47 & $8.99 \%$ & $30.12 \%$ & $1.70 E+21$ & $3.78 \mathrm{E}+14$ \\
\hline A2 & L1P256 & 684.15 & 11207.49 & 145.14 & $7.45 \%$ & $24.96 \%$ & $1.64 \mathrm{E}+21$ & $3.65 \mathrm{E}+14$ \\
\hline A3 & L2P15Z & 340.31 & 5304.89 & 144.82 & $7.55 \%$ & $12.60 \%$ & $7.78 \mathrm{E}+20$ & $1.73 E+14$ \\
\hline A4 & L1P135 & 820.48 & 13486.86 & 176.68 & $8.88 \%$ & $29.75 \%$ & $1.98 \mathrm{E}+21$ & $4.41 \mathrm{E}+14$ \\
\hline A5 & L1P234 & 949.02 & 15214.06 & 210.71 & $10.35 \%$ & $34.67 \%$ & $2.29 \mathrm{E}+21$ & $5.10 \mathrm{E}+14$ \\
\hline A6 & L1P213 & 788.96 & 12854.57 & 168.39 & $8.66 \%$ & $29.01 \%$ & $1.91 \mathrm{E}+21$ & $4.25 \mathrm{E}+14$ \\
\hline A7 & L1P192 & 797.99 & 13213.14 & 177.72 & $8.71 \%$ & $29.18 \%$ & $1.96 \mathrm{E}+21$ & $4.36 \mathrm{E}+14$ \\
\hline A8 & L1P171 & 999.24 & 16128.87 & 208.87 & $10.79 \%$ & $36.15 \%$ & $2.41 \mathrm{E}+21$ & $5.36 \mathrm{E}+14$ \\
\hline B1 & Blank & -- & -- & -- & -- & -- & -- & -- \\
\hline B2 & L1F401 & -- & -- & -- & -- & -- & -- & -- \\
\hline B3 & L1F417 & -- & -- & -- & -- & -- & -- & -- \\
\hline B4 & Blank & -- & -- & -- & -- & -- & -- & -- \\
\hline B5 & Blank & -- & -- & -- & -- & -- & -- & -- \\
\hline $\mathrm{B} 6$ & L2F46Z & -- & -- & -- & -- & -- & -- & -- \\
\hline B7 & L1F44N & -- & -- & -- & -- & -- & -- & -- \\
\hline B8 & Blank & -- & -- & -- & -- & -- & -- & -- \\
\hline $\mathrm{C} 1$ & L1P12Z & 1175.14 & 14868.69 & 230.46 & $12.63 \%$ & $42.31 \%$ & $2.32 \mathrm{E}+21$ & $5.16 \mathrm{E}+14$ \\
\hline $\mathrm{C} 2$ & L1P266 & 956.51 & 14999.26 & 194.24 & $10.64 \%$ & $35.64 \%$ & $2.30 \mathrm{E}+21$ & $5.12 \mathrm{E}+14$ \\
\hline $\mathrm{C} 3$ & L2P16Z & 484.92 & 7190.58 & 192.71 & $11.03 \%$ & $18.40 \%$ & $1.09 \mathrm{E}+21$ & $2.43 \mathrm{E}+14$ \\
\hline C4 & L1P145 & 1152.20 & 18142.24 & 242.20 & $12.66 \%$ & $42.41 \%$ & $2.79 \mathrm{E}+21$ & $6.21 \mathrm{E}+14$ \\
\hline $\mathrm{C} 5$ & L1P244 & 1092.63 & 17404.31 & 229.74 & $12.09 \%$ & $40.50 \%$ & $2.69 \mathrm{E}+21$ & $5.99 \mathrm{E}+14$ \\
\hline $\mathrm{C} 6$ & L1P223 & 894.78 & 14554.25 & 192.12 & $9.99 \%$ & $33.47 \%$ & $2.23 \mathrm{E}+21$ & $4.96 \mathrm{E}+14$ \\
\hline $\mathrm{C} 7$ & L1P202 & 914.03 & 14134.78 & 196.47 & $10.06 \%$ & $33.70 \%$ & $2.13 \mathrm{E}+21$ & $4.74 \mathrm{E}+14$ \\
\hline C8 & L1P181 & 1139.17 & 17977.59 & 237.30 & $12.37 \%$ & $41.44 \%$ & $2.73 \mathrm{E}+21$ & $6.08 \mathrm{E}+14$ \\
\hline D1 & Blank & -- & -- & -- & -- & -- & -- & -- \\
\hline $\mathrm{D} 2$ & L1F381 & -- & -- & -- & -- & -- & -- & -- \\
\hline D3 & L1F4127 & -- & -- & -- & -- & -- & -- & -- \\
\hline D4 & Blank & -- & -- & -- & -- & -- & -- & -- \\
\hline D5 & Blank & -- & -- & -- & -- & -- & -- & -- \\
\hline $\mathrm{D} 6$ & L2F45Z & -- & -- & -- & -- & -- & -- & -- \\
\hline D7 & L2F47Z & -- & -- & -- & -- & -- & -- & -- \\
\hline $\mathrm{D} 8$ & Blank & -- & -- & -- & -- & -- & -- & -- \\
\hline
\end{tabular}


Table 10. Cycle 143A 02 average plate power and burnup.

\begin{tabular}{|c|c|c|c|c|c|c|c|c|}
\hline \multirow[b]{2}{*}{$\begin{array}{c}\text { Position } \\
\text { ID }\end{array}$} & \multirow[b]{2}{*}{ Plate ID } & \multicolumn{7}{|c|}{ 143A 15.0 EFPD } \\
\hline & & $\begin{array}{l}\text { Fission } \\
\text { Heat } \\
\text { Rate } \\
(\mathrm{W} / \mathrm{g})\end{array}$ & $\begin{array}{l}\text { Fission } \\
\text { Power } \\
\text { Density } \\
\text { (w/cm3) }\end{array}$ & $\begin{array}{l}\text { Heat } \\
\text { Flux } \\
(\mathrm{w} / \mathrm{cm} 2)\end{array}$ & $\begin{array}{c}\text { U-235 Burnup } \\
\text { u-235/u- } \\
\text { 235initial (\%) }\end{array}$ & $\begin{array}{c}\text { LEUeq } \\
\text { Burnup } \\
(\%)\end{array}$ & $\begin{array}{c}\text { Fission } \\
\text { Density } \\
(\mathrm{f} / \mathrm{cc})\end{array}$ & $\begin{array}{l}\text { Fission } \\
\text { Rate } \\
\text { (f/cc-s) }\end{array}$ \\
\hline $\mathrm{A} 1$ & L1P30Z & 1100.79 & 15009.19 & 219.13 & $12.57 \%$ & $42.11 \%$ & $2.39 E+21$ & $1.84 \mathrm{E}+15$ \\
\hline $\mathrm{A} 2$ & L1P256 & 924.16 & 14914.49 & 193.14 & $10.50 \%$ & $35.18 \%$ & $2.32 \mathrm{E}+21$ & $1.79 \mathrm{E}+15$ \\
\hline A3 & L2P15Z & 464.21 & 7182.75 & 196.09 & $10.66 \%$ & $17.79 \%$ & $1.10 \mathrm{E}+21$ & $8.49 \mathrm{E}+14$ \\
\hline A4 & L1P135 & 1138.27 & 18373.72 & 240.70 & $12.66 \%$ & $42.41 \%$ & $2.82 \mathrm{E}+21$ & $2.18 \mathrm{E}+15$ \\
\hline A5 & L1P234 & 1334.04 & 20928.28 & 289.86 & $14.69 \%$ & $49.21 \%$ & $3.25 \mathrm{E}+21$ & $2.51 \mathrm{E}+15$ \\
\hline A6 & L1P213 & 1124.03 & 17985.55 & 235.61 & $12.29 \%$ & $41.17 \%$ & $2.72 \mathrm{E}+21$ & $2.10 \mathrm{E}+15$ \\
\hline A7 & L1P192 & 1128.95 & 18355.72 & 246.88 & $12.39 \%$ & $41.51 \%$ & $2.79 \mathrm{E}+21$ & $2.15 E+15$ \\
\hline A8 & L1P171 & 1397.32 & 22046.38 & 285.50 & $15.32 \%$ & $51.32 \%$ & $3.43 \mathrm{E}+21$ & $2.65 E+15$ \\
\hline B1 & Blank & $\overline{--}$ & $\overline{--}$ & - & $\overline{--}$ & $\overline{--}$ & $\overline{--}$ & $\overline{--}$ \\
\hline B2 & & 1891.78 & 29712.48 & 365.46 & $67 \%$ & $22.34 \%$ & $1.45 \mathrm{E}+21$ & $1.12 \mathrm{E}+15$ \\
\hline B3 & L1F417 & 1897.60 & 29316.37 & 372.32 & $71 \%$ & $22.48 \%$ & $1.43 \mathrm{E}+21$ & $1.10 \mathrm{E}+15$ \\
\hline B4 & Blank & -- & -- & -- & -- & -- & -- & -- \\
\hline B5 & $\mathrm{B}$ & -- & -- & -- & -- & -- & 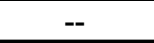 & -- \\
\hline $\mathrm{B} 6$ & $46 Z$ & 1016.01 & 14603.54 & 368.74 & $35 \%$ & $12.16 \%$ & $7.24 \mathrm{E}+20$ & $5.59 \mathrm{E}+14$ \\
\hline B7 & IF44N & 2068.96 & 27642.50 & 382.85 & $7.35 \%$ & $24.62 \%$ & $1.38 \mathrm{E}+21$ & $1.06 \mathrm{E}+15$ \\
\hline $\mathrm{B} 8$ & ank & -- & -- & -- & -- & - & -- & -- \\
\hline $\mathrm{C} 1$ & L1P12Z & 1730.80 & 21271.59 & 329.71 & $.18 \%$ & $60.90 \%$ & $3.34 \mathrm{E}+21$ & $2.58 \mathrm{E}+15$ \\
\hline $\mathrm{C} 2$ & $11 \mathrm{P} 266$ & 1411.11 & 21613.49 & 279.89 & $36 \%$ & $51.46 \%$ & $3.33 E+21$ & $2.57 E+15$ \\
\hline C3 & L2P16Z & 700.00 & 10259.28 & 274.95 & $79 \%$ & $26.35 \%$ & $1.58 \mathrm{E}+21$ & $1.22 \mathrm{E}+15$ \\
\hline C4 & L1P145 & 1719.43 & 26310.81 & 351.25 & $18.28 \%$ & $61.24 \%$ & $4.05 \mathrm{E}+21$ & $3.13 E+15$ \\
\hline C5 & L1P244 & 1583.06 & 24560.79 & 324.20 & $17.31 \%$ & $57.99 \%$ & $3.86 \mathrm{E}+21$ & $2.98 \mathrm{E}+15$ \\
\hline C6 & L1P223 & 1316.51 & 20952.04 & 276.57 & $14.40 \%$ & $48.24 \%$ & $3.22 \mathrm{E}+21$ & $2.48 \mathrm{E}+15$ \\
\hline $\mathrm{C} 7$ & L1P202 & 1337.17 & 20223.16 & 281.10 & $14.54 \%$ & $48.71 \%$ & $3.09 \mathrm{E}+21$ & $2.38 \mathrm{E}+15$ \\
\hline $\mathrm{C} 8$ & L1P181 & 1651.65 & 25359.69 & 334.75 & $17.79 \%$ & $59.60 \%$ & $3.95 \mathrm{E}+21$ & $3.05 E+15$ \\
\hline $\mathrm{D} 1$ & Blank & -- & -- & - & -- & -- & - & - \\
\hline D2 & L1F38 & 1718.07 & 27846.18 & 339.72 & $6.10 \%$ & $20.44 \%$ & $1.35 \mathrm{E}+21$ & $1.04 \mathrm{E}+15$ \\
\hline D3 & L1F427 & 1757.01 & 28450.25 & 347.09 & $6.10 \%$ & $20.44 \%$ & $1.36 \mathrm{E}+21$ & $1.05 \mathrm{E}+15$ \\
\hline$\overline{D 4}$ & & $\overline{--}$ & -- & $\overline{--}$ & - & -- & $\overline{--}$ & $\overline{--}$ \\
\hline D5 & & -- & -- & $\overline{--}$ & - & - & $\overline{--}$ & -- \\
\hline $\mathrm{D} 6$ & 452 & 647.07 & 14050.07 & 340.71 & $7 \%$ & $7.63 \%$ & $6.65 \mathrm{E}+20$ & $5.13 E+14$ \\
\hline D7 & L2F47Z & 743.47 & 11119.70 & 274.10 & $5.25 \%$ & $8.76 \%$ & $5.24 \mathrm{E}+20$ & $4.04 \mathrm{E}+14$ \\
\hline D8 & Blank & -- & - & -- & -- & - & 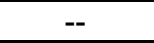 & - \\
\hline
\end{tabular}


Table 11. Cycle 143A 03 average plate power burnup.

\begin{tabular}{|c|c|c|c|c|c|c|c|c|}
\hline \multirow[b]{2}{*}{ Position ID } & \multirow[b]{2}{*}{ Plate ID } & \multicolumn{7}{|c|}{ 143A 26.0 EFPD } \\
\hline & & $\begin{array}{l}\text { Fission } \\
\text { Heat } \\
\text { Rate } \\
(\mathrm{W} / \mathrm{g})\end{array}$ & $\begin{array}{l}\text { Fission } \\
\text { Power } \\
\text { Density } \\
(w / c m 3)\end{array}$ & $\begin{array}{l}\text { Heat Flux } \\
(\mathrm{w} / \mathrm{cm} 2)\end{array}$ & $\begin{array}{c}\text { U-235 Burnup } \\
\text { u-235/u- } \\
\text { 235initial (\%) }\end{array}$ & $\begin{array}{l}\text { LEUeq } \\
\text { Burnup } \\
(\%)\end{array}$ & $\begin{array}{l}\text { Fission } \\
\text { Density } \\
\text { (f/cc) }\end{array}$ & $\begin{array}{l}\text { Fission } \\
\text { Rate (f/cc } \\
\text { s) }\end{array}$ \\
\hline $\mathrm{A} 1$ & L1P30Z & 1099.96 & 14806.39 & 216.17 & $15.17 \%$ & $50.82 \%$ & $2.88 \mathrm{E}+21$ & $1.28 \mathrm{E}+15$ \\
\hline $\mathrm{A} 2$ & L1P256 & 928.89 & 14828.31 & 192.03 & $12.72 \%$ & $42.61 \%$ & $2.81 \mathrm{E}+21$ & $1.25 \mathrm{E}+15$ \\
\hline A3 & L2P15Z & 463.15 & 7126.35 & 194.55 & $12.88 \%$ & $21.49 \%$ & $1.34 \mathrm{E}+21$ & $5.97 \mathrm{E}+14$ \\
\hline A4 & L1P135 & 1136.69 & 18104.54 & 237.17 & $15.33 \%$ & $51.36 \%$ & $3.42 \mathrm{E}+21$ & $1.52 E+15$ \\
\hline A5 & L1P234 & 1331.66 & 20568.00 & 284.87 & $17.77 \%$ & $59.53 \%$ & $3.94 \mathrm{E}+21$ & $1.75 \mathrm{E}+15$ \\
\hline A6 & L1P213 & 1129.86 & 17842.64 & 233.74 & $14.96 \%$ & $50.12 \%$ & $3.31 \mathrm{E}+21$ & $1.47 E+15$ \\
\hline A7 & L1P192 & 1136.23 & 18229.56 & 245.19 & $15.05 \%$ & $50.42 \%$ & $3.39 \mathrm{E}+21$ & $1.51 \mathrm{E}+15$ \\
\hline A8 & L1P171 & 1396.15 & 21670.62 & 280.63 & $18.52 \%$ & $62.04 \%$ & $4.15 \mathrm{E}+21$ & $1.85 \mathrm{E}+15$ \\
\hline $\mathrm{B} 1$ & Blank & -- & -- & -- & -- & -- & -- & -- \\
\hline $\mathrm{B} 2$ & L1F401 & 1892.10 & 29075.16 & 357.62 & $11.15 \%$ & $37.35 \%$ & $2.42 \mathrm{E}+21$ & $1.08 \mathrm{E}+15$ \\
\hline B3 & L1F417 & 1898.52 & 28688.84 & 364.35 & $11.22 \%$ & $37.59 \%$ & $2.39 \mathrm{E}+21$ & $1.06 \mathrm{E}+15$ \\
\hline B4 & Blank & -- & -- & -- & -- & -- & -- & -- \\
\hline B5 & Blank & -- & -- & -- & -- & -- & -- & -- \\
\hline B6 & L2F46Z & 1004.61 & 14269.85 & 360.31 & $12.34 \%$ & $20.42 \%$ & $1.20 \mathrm{E}+21$ & $5.34 \mathrm{E}+14$ \\
\hline B7 & L1F44N & 2066.83 & 26963.40 & 373.44 & $12.26 \%$ & $41.07 \%$ & $2.28 \mathrm{E}+21$ & $1.01 \mathrm{E}+15$ \\
\hline B8 & Blank & -- & -- & -- & -- & -- & -- & -- \\
\hline C1 & L1P12Z & 1729.95 & 20833.42 & 322.92 & $21.96 \%$ & $73.57 \%$ & $4.04 \mathrm{E}+21$ & $1.80 \mathrm{E}+15$ \\
\hline $\mathrm{C} 2$ & L1P266 & 1420.50 & 21400.87 & 277.14 & $18.62 \%$ & $62.38 \%$ & $4.03 \mathrm{E}+21$ & $1.79 \mathrm{E}+15$ \\
\hline C3 & L2P16Z & 698.60 & 10153.41 & 272.11 & $19.14 \%$ & $31.94 \%$ & $1.91 \mathrm{E}+21$ & $8.50 \mathrm{E}+14$ \\
\hline $\mathrm{C} 4$ & L1P145 & 1714.03 & 25701.43 & 343.11 & $22.15 \%$ & $74.20 \%$ & $4.91 \mathrm{E}+21$ & $2.19 \mathrm{E}+15$ \\
\hline C5 & L1P244 & 1579.83 & 24057.65 & 317.56 & $20.84 \%$ & $69.81 \%$ & $4.67 \mathrm{E}+21$ & $2.08 \mathrm{E}+15$ \\
\hline $\mathrm{C6}$ & L1P223 & 1325.31 & 20766.44 & 274.12 & $17.44 \%$ & $58.42 \%$ & $3.90 \mathrm{E}+21$ & $1.74 \mathrm{E}+15$ \\
\hline $\mathrm{C} 7$ & L1P202 & 1346.40 & 20049.97 & 278.69 & $17.61 \%$ & $58.99 \%$ & $3.75 \mathrm{E}+21$ & $1.67 \mathrm{E}+15$ \\
\hline $\mathrm{C} 8$ & L1P181 & 1646.89 & 24802.49 & 327.39 & $21.54 \%$ & $72.16 \%$ & $4.78 \mathrm{E}+21$ & $2.13 \mathrm{E}+15$ \\
\hline D1 & Blank & -- & -- & -- & -- & -- & -- & -- \\
\hline D2 & L1F381 & 1719.78 & 27320.47 & 333.31 & $10.21 \%$ & $34.20 \%$ & $2.26 \mathrm{E}+21$ & $1.01 \mathrm{E}+15$ \\
\hline D3 & L1F427 & 1757.60 & 27885.63 & 340.20 & $10.30 \%$ & $34.51 \%$ & $2.29 \mathrm{E}+21$ & $1.02 \mathrm{E}+15$ \\
\hline D4 & Blank & -- & -- & -- & -- & -- & -- & -- \\
\hline D5 & Blank & -- & -- & -- & -- & -- & -- & -- \\
\hline D6 & L2F45Z & 644.52 & 13888.14 & 336.79 & $7.83 \%$ & $13.06 \%$ & $1.12 \mathrm{E}+21$ & $4.99 \mathrm{E}+14$ \\
\hline D7 & L2F47Z & 736.74 & 10924.97 & 269.30 & $8.86 \%$ & $14.78 \%$ & $8.87 \mathrm{E}+20$ & $3.95 \mathrm{E}+14$ \\
\hline D8 & Blank & -- & -- & -- & -- & -- & -- & -- \\
\hline
\end{tabular}


Table 12. Cycle 143A 04 average plate power burnup.

\begin{tabular}{|c|c|c|c|c|c|c|c|c|}
\hline \multirow[b]{2}{*}{ Position ID } & \multirow[b]{2}{*}{ Plate ID } & \multicolumn{7}{|c|}{ 143A 37.0 EFPD } \\
\hline & & $\begin{array}{c}\text { Fission } \\
\text { Heat } \\
\text { Rate } \\
(\mathrm{W} / \mathrm{g})\end{array}$ & $\begin{array}{l}\text { Fission } \\
\text { Power } \\
\text { Density } \\
\text { (w/cm3) }\end{array}$ & $\begin{array}{l}\text { Heat Flux } \\
(\mathrm{w} / \mathrm{cm} 2)\end{array}$ & $\begin{array}{c}\text { U-235 Burnup } \\
\text { u-235/u- } \\
\text { 235initial (\%) }\end{array}$ & $\begin{array}{l}\text { LEUeq } \\
\text { Burnup } \\
(\%)\end{array}$ & $\begin{array}{l}\text { Fission } \\
\text { Density } \\
\text { (f/cc) }\end{array}$ & $\begin{array}{l}\text { Fission } \\
\text { Rate (f/cc- } \\
\text { s) }\end{array}$ \\
\hline A1 & L1P30Z & -- & -- & -- & -- & -- & -- & -- \\
\hline $\mathrm{A} 2$ & L1P256 & -- & -- & -- & -- & -- & -- & -- \\
\hline A3 & L2P15Z & -- & -- & -- & -- & -- & -- & -- \\
\hline A4 & L1P135 & -- & -- & -- & -- & -- & -- & -- \\
\hline A5 & L1P234 & -- & -- & -- & -- & -- & -- & -- \\
\hline A6 & L1P213 & -- & -- & -- & -- & -- & -- & -- \\
\hline A7 & L1P192 & -- & -- & -- & -- & -- & -- & -- \\
\hline A8 & L1P171 & - & - & -- & -- & - & -- & -- \\
\hline $\mathrm{B} 1$ & Blank & -- & -- & -- & -- & -- & -- & -- \\
\hline B2 & L1F401 & 2098.77 & 31470.48 & 387.09 & $16.03 \%$ & $53.70 \%$ & $3.47 E+21$ & $1.09 \mathrm{E}+15$ \\
\hline B3 & L1F417 & 2192.97 & 32299.29 & 410.20 & $16.31 \%$ & $54.64 \%$ & $3.47 E+21$ & $1.09 \mathrm{E}+15$ \\
\hline B4 & Blank & -- & -- & -- & -- & -- & -- & -- \\
\hline B5 & Blank & -- & -- & -- & -- & -- & -- & -- \\
\hline B6 & L2F46Z & 1090.63 & 15295.55 & 386.21 & $17.58 \%$ & $29.09 \%$ & $1.71 \mathrm{E}+21$ & $5.35 \mathrm{E}+14$ \\
\hline $\mathrm{B} 7$ & L1F44N & 2351.60 & 29844.92 & 413.35 & $17.69 \%$ & $59.26 \%$ & $3.28 \mathrm{E}+21$ & $1.03 E+15$ \\
\hline B8 & Blank & -- & -- & -- & -- & -- & -- & -- \\
\hline $\mathrm{C} 1$ & L1P12Z & -- & -- & -- & -- & -- & -- & -- \\
\hline $\mathrm{C} 2$ & L1P266 & -- & -- & -- & -- & -- & -- & -- \\
\hline C3 & L2P16Z & -- & -- & -- & -- & -- & -- & -- \\
\hline $\mathrm{C} 4$ & L1P145 & -- & -- & -- & -- & -- & -- & -- \\
\hline C5 & L1P244 & -- & -- & -- & -- & -- & -- & -- \\
\hline $\mathrm{C6}$ & L1P223 & -- & -- & -- & -- & -- & -- & -- \\
\hline $\mathrm{C} 7$ & L1P202 & -- & -- & -- & -- & -- & -- & -- \\
\hline $\mathrm{C} 8$ & L1P181 & -- & - & -- & -- & -- & -- & -- \\
\hline D1 & Blank & -- & -- & -- & -- & -- & -- & -- \\
\hline $\mathrm{D} 2$ & L1F381 & 1945.30 & 30210.41 & 368.57 & $14.76 \%$ & $49.45 \%$ & $3.27 E+21$ & $1.02 \mathrm{E}+15$ \\
\hline D3 & L1F427 & 2039.90 & 31604.56 & 385.58 & $15.09 \%$ & $50.55 \%$ & $3.35 E+21$ & $1.05 \mathrm{E}+15$ \\
\hline $\mathrm{D} 4$ & Blank & -- & -- & -- & -- & -- & -- & -- \\
\hline D5 & Blank & -- & -- & -- & -- & -- & -- & -- \\
\hline D6 & L2F45Z & 729.75 & 15591.28 & 378.09 & $11.33 \%$ & $18.90 \%$ & $1.64 \mathrm{E}+21$ & $5.13 \mathrm{E}+14$ \\
\hline D7 & L2F47Z & 858.90 & 12608.23 & 310.79 & $13.07 \%$ & $21.81 \%$ & $1.31 \mathrm{E}+21$ & $4.10 \mathrm{E}+14$ \\
\hline D8 & Blank & -- & -- & -- & -- & -- & -- & -- \\
\hline
\end{tabular}


Table 13. Cycle 143A 05 (EOC) average plate power and burnup.

\begin{tabular}{|c|c|c|c|c|c|c|c|c|}
\hline \multirow[b]{2}{*}{ Position ID } & \multirow[b]{2}{*}{ Plate ID } & \multicolumn{7}{|c|}{ 143A 48.9 EFPD (EOC) } \\
\hline & & $\begin{array}{c}\text { Fission } \\
\text { Heat } \\
\text { Rate } \\
(\mathrm{W} / \mathrm{g})\end{array}$ & $\begin{array}{l}\text { Fission } \\
\text { Power } \\
\text { Density } \\
\text { (w/cm3) }\end{array}$ & $\begin{array}{c}\text { Heat Flux } \\
(\mathrm{w} / \mathrm{cm} 2)\end{array}$ & $\begin{array}{c}\text { U-235 Burnup } \\
\text { u-235/u- } \\
\text { 235initial (\%) }\end{array}$ & $\begin{array}{l}\text { LEUeq } \\
\text { Burnup } \\
(\%)\end{array}$ & $\begin{array}{l}\text { Fission } \\
\text { Density } \\
(\mathrm{f} / \mathrm{cc})\end{array}$ & $\begin{array}{c}\text { Fission } \\
\text { Rate (f/cc- } \\
\mathrm{s})\end{array}$ \\
\hline $\mathrm{A} 1$ & L1P30Z & -- & -- & -- & -- & -- & -- & -- \\
\hline $\mathrm{A} 2$ & L1P256 & -- & -- & -- & -- & -- & -- & -- \\
\hline A3 & L2P15Z & -- & -- & -- & -- & -- & -- & -- \\
\hline A4 & L1P135 & -- & -- & -- & -- & -- & -- & -- \\
\hline A5 & L1P234 & -- & -- & -- & -- & -- & -- & -- \\
\hline A6 & L1P213 & -- & -- & -- & -- & -- & -- & -- \\
\hline A7 & L1P192 & -- & -- & -- & -- & -- & -- & -- \\
\hline A8 & L1P171 & -- & -- & -- & -- & -- & -- & -- \\
\hline B1 & Blank & -- & -- & -- & -- & -- & -- & -- \\
\hline B2 & L1F401 & 2096.89 & 30619.84 & 376.62 & $21.14 \%$ & $70.82 \%$ & $4.58 \mathrm{E}+21$ & $1.08 \mathrm{E}+15$ \\
\hline B3 & L1F417 & 2184.18 & 31295.20 & 397.45 & $21.63 \%$ & $72.46 \%$ & $4.61 \mathrm{E}+21$ & $1.09 \mathrm{E}+15$ \\
\hline B4 & Blank & -- & -- & -- & -- & -- & -- & -- \\
\hline B5 & Blank & -- & -- & -- & -- & -- & -- & -- \\
\hline B6 & L2F46Z & 1072.37 & 14835.00 & 374.58 & $23.09 \%$ & $38.21 \%$ & $2.25 E+21$ & $5.33 \mathrm{E}+14$ \\
\hline B7 & L1F44N & 2341.33 & 28846.91 & 399.53 & $23.30 \%$ & $78.06 \%$ & $4.34 \mathrm{E}+21$ & $1.03 \mathrm{E}+15$ \\
\hline B8 & Blank & -- & -- & -- & -- & -- & -- & -- \\
\hline C1 & L1P12Z & -- & -- & -- & -- & -- & -- & -- \\
\hline $\mathrm{C} 2$ & L1P266 & -- & -- & -- & -- & -- & -- & -- \\
\hline C3 & L2P16Z & -- & -- & -- & -- & -- & -- & -- \\
\hline $\mathrm{C} 4$ & L1P145 & -- & -- & -- & -- & -- & -- & -- \\
\hline $\mathrm{C5}$ & L1P244 & -- & -- & -- & -- & -- & -- & -- \\
\hline C6 & L1P223 & -- & -- & -- & -- & -- & -- & -- \\
\hline $\mathrm{C} 7$ & L1P202 & -- & -- & -- & -- & -- & -- & -- \\
\hline C8 & L1P181 & -- & -- & -- & -- & -- & -- & -- \\
\hline D1 & Blank & -- & -- & -- & -- & -- & -- & -- \\
\hline D2 & L1F381 & 1946.23 & 29488.28 & 359.76 & $19.53 \%$ & $65.43 \%$ & $4.33 E+21$ & $1.02 \mathrm{E}+15$ \\
\hline D3 & L1F427 & 2035.30 & 30729.74 & 374.90 & $20.06 \%$ & $67.20 \%$ & $4.47 E+21$ & $1.06 \mathrm{E}+15$ \\
\hline D4 & Blank & -- & -- & -- & -- & -- & -- & -- \\
\hline D5 & Blank & -- & -- & -- & -- & -- & -- & -- \\
\hline D6 & L2F45Z & 724.63 & 15339.81 & 371.99 & $15.12 \%$ & $25.23 \%$ & $2.19 \mathrm{E}+21$ & $5.18 \mathrm{E}+14$ \\
\hline D7 & L2F47Z & 846.70 & 12293.89 & 303.04 & $17.46 \%$ & $29.13 \%$ & $1.75 \mathrm{E}+21$ & $4.14 \mathrm{E}+14$ \\
\hline D8 & Blank & -- & -- & -- & -- & -- & -- & -- \\
\hline
\end{tabular}




\subsection{Gradients}

The MCNP-calculated power gradients in the transverse and axial directions are represented by the thermal neutron flux and fission rate local-2-average ratios (L2ARs) as a function of position along the plate. Figures 1 and 2 depict the power gradient in the transverse direction and Figures 3 and 4 depict the power gradient in the axial direction.

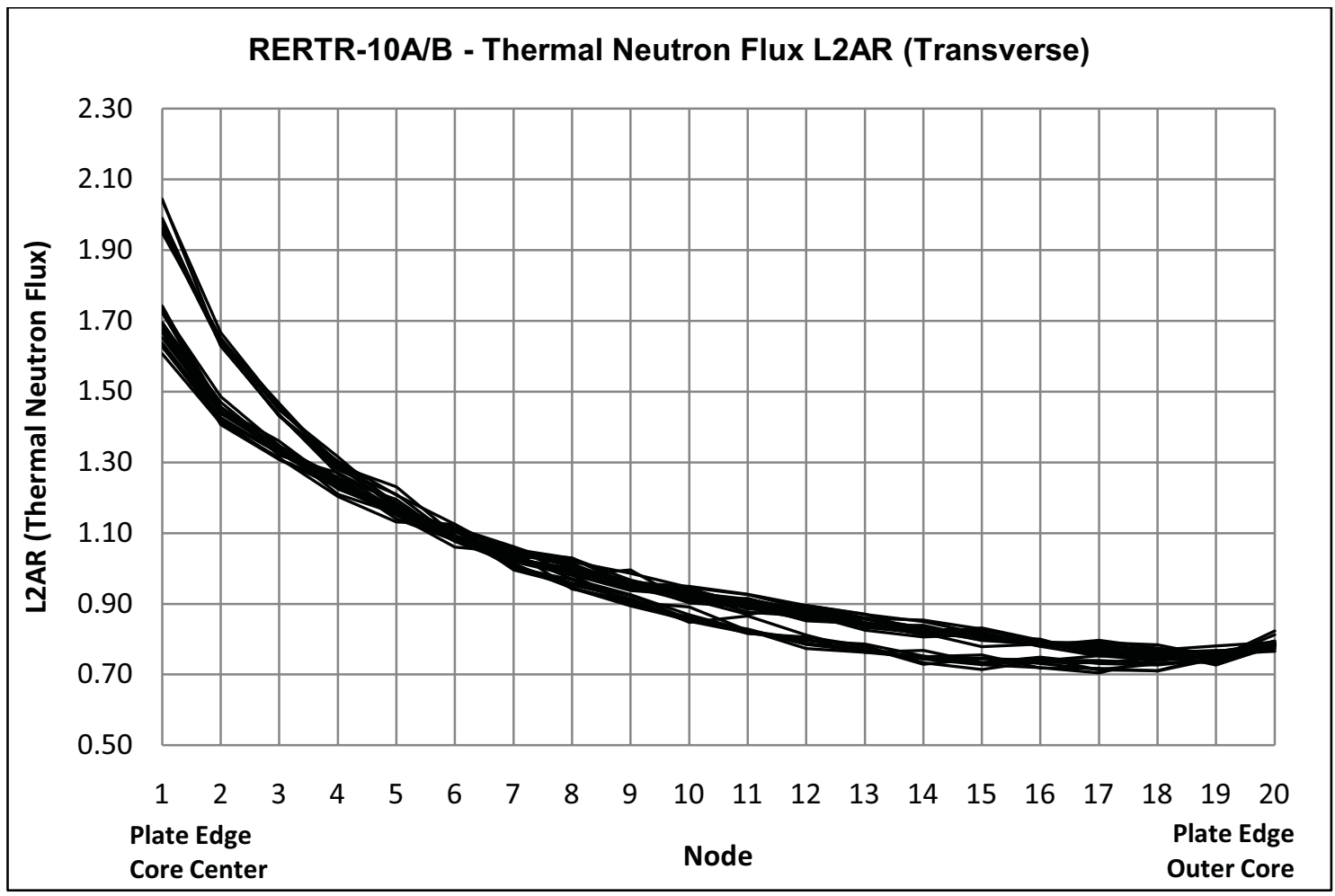

Figure 5. RERTR-10 fuel meat thermal neutron flux L2ARs in transverse direction. ${ }^{6}$ 


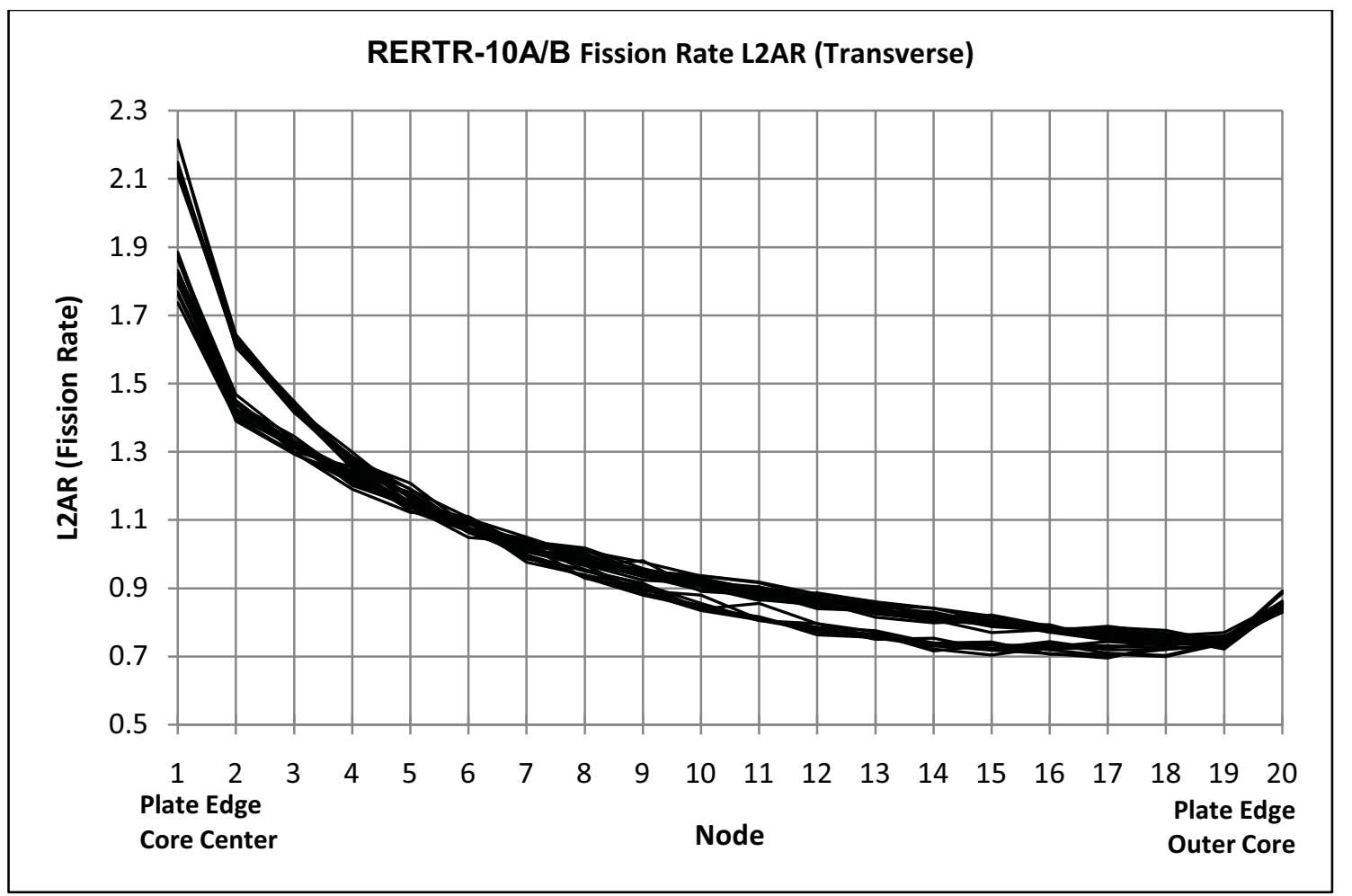

Figure 6. RERTR-10 fuel meat fission rate L2ARs in transverse direction. ${ }^{6}$

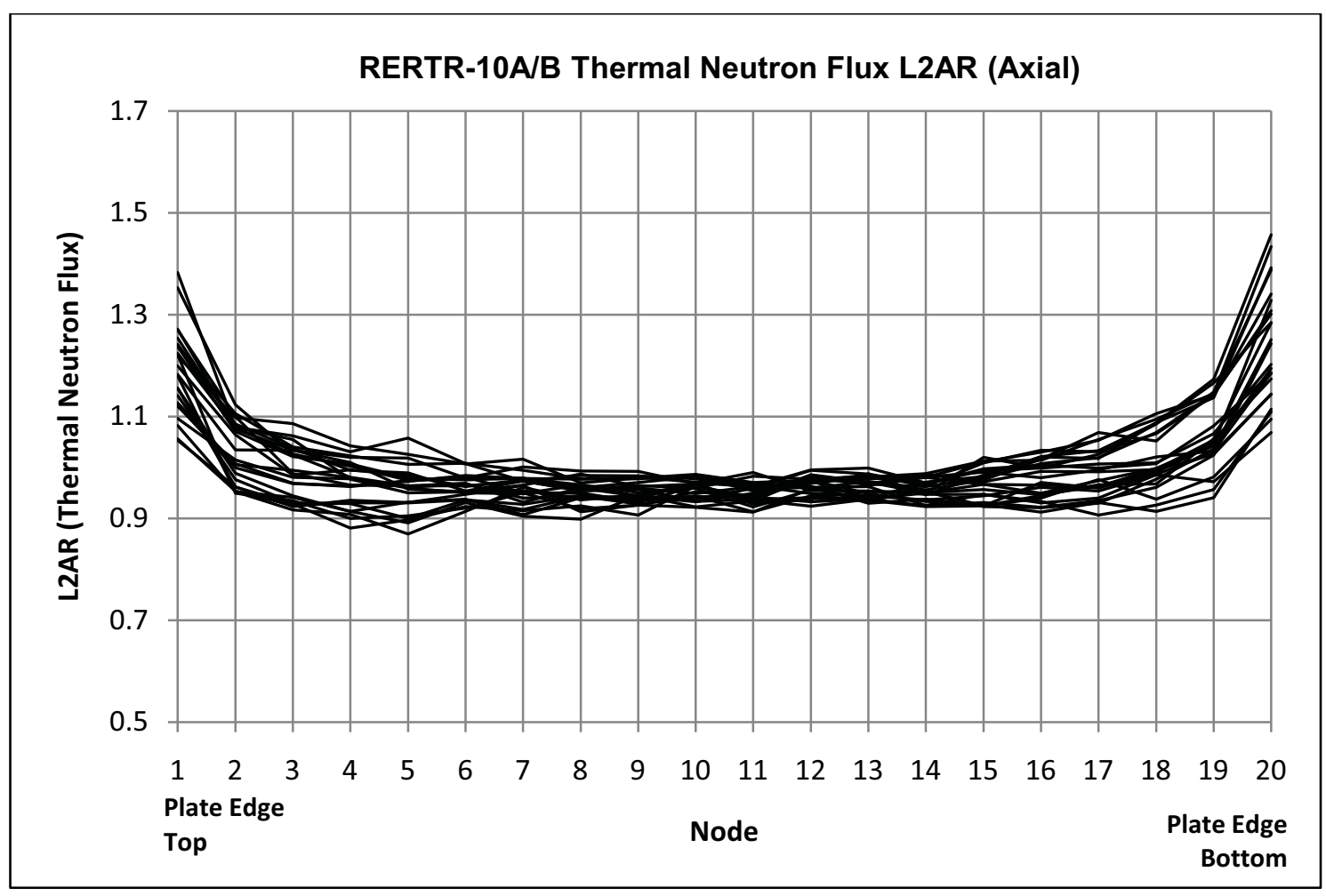

Figure 7. RERTR-10 fuel meat thermal neutron flux L2ARs in axial direction. ${ }^{6}$ 


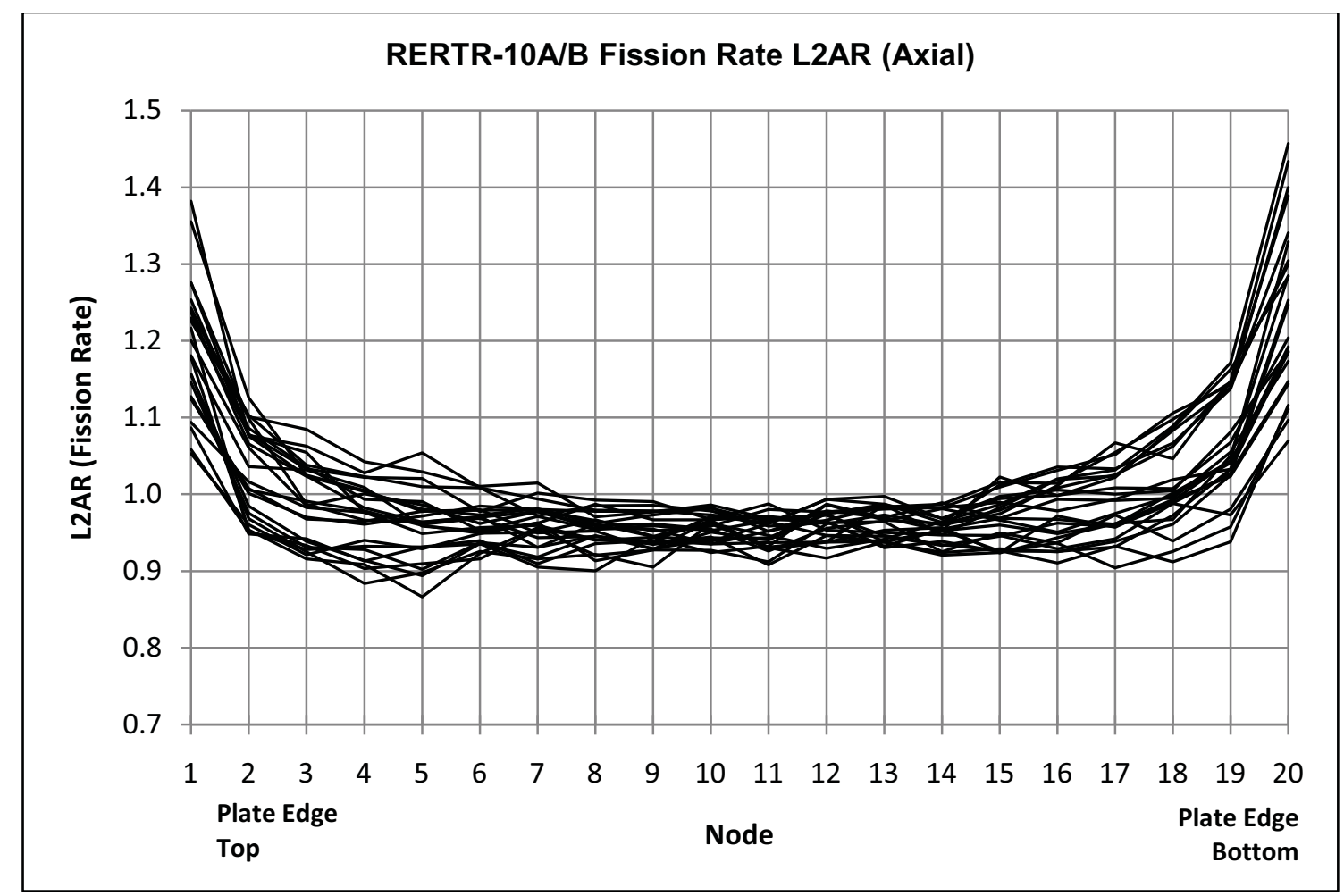

Figure 8. RERTR-10 fuel meat fission rate L2ARs in axial direction. ${ }^{6}$ 


\section{HYDRAULIC TESTING}

A fully assembled irradiation test vehicle (with simulated fuel plates) was used for testing. The test vehicle was fabricated such that the orifice plates could be easily changed. The hydraulic resistance of the RERTR Large B-Position irradiation test vehicle with various orifice plate sizes were calculated, the results are shown in Table 14.

Table 14. Loss coefficients for the RERTR Irradiation Test Vehicle components. ${ }^{7}$

\begin{tabular}{ccc}
\hline $\begin{array}{c}\text { Orifice Dia. } \\
(\mathrm{mm})\end{array}$ & $\begin{array}{c}\mathrm{K} / \mathrm{A}^{2} \\
\left(1 / \mathrm{m}^{4}\right)\end{array}$ & $\begin{array}{c}\text { ATR Coolant } \\
\text { Flow Rate } \\
\left(\mathrm{cm}^{3} / \mathrm{sec}\right)\end{array}$ \\
\hline 10 & $5.3041 \times 10^{8}$ & 1252 \\
9 & $8.2181 \times 10^{8}$ & 1046 \\
8 & $1.6961 \times 10^{9}$ & 757 \\
7.32 & $2.9022 \times 10^{9}$ & 588 \\
7 & $3.0058 \times 10^{9}$ & 579 \\
6 & $4.0784 \times 10^{9}$ & 500 \\
5 & $101743 \times 10^{10}$ & 298 \\
\hline Bypass & $2.7958 \times 10^{8}$ & -- \\
Vehicle & $1.4161 \times 10^{8}$ & 2727 \\
\hline
\end{tabular}

Based on the results from the hydraulic testing, the orifice was removed leaving the capsule in the "Vehicle" configuration to provide an ATR coolant flow rate through the capsules of $2727 \mathrm{~cm}^{3} / \mathrm{sec}^{8}$ 


\section{AS-RUN THERMAL ANALYSIS}

The MCNP-calculated heat flux, nominal coolant flow rate, as-built plate thickness and ABAQUS were used to calculate the coolant and plate surface temperatures.

\subsection{Coolant Temperature as a function of Location}

The coolant temperature was analyzed at the five flow channels in the test assembly, with Channel 1 at the right of the assembly. For each cycle interval, the coolant temperature was plotted as a function of location along the test assembly with 0 inches being at the top of the assembly. These plots are show in Figure 9 through Figure 17.



Figure 9. Coolant temperature as a function of location along the test assembly for Cycle 142B 01 (BOC). ${ }^{9}$ 


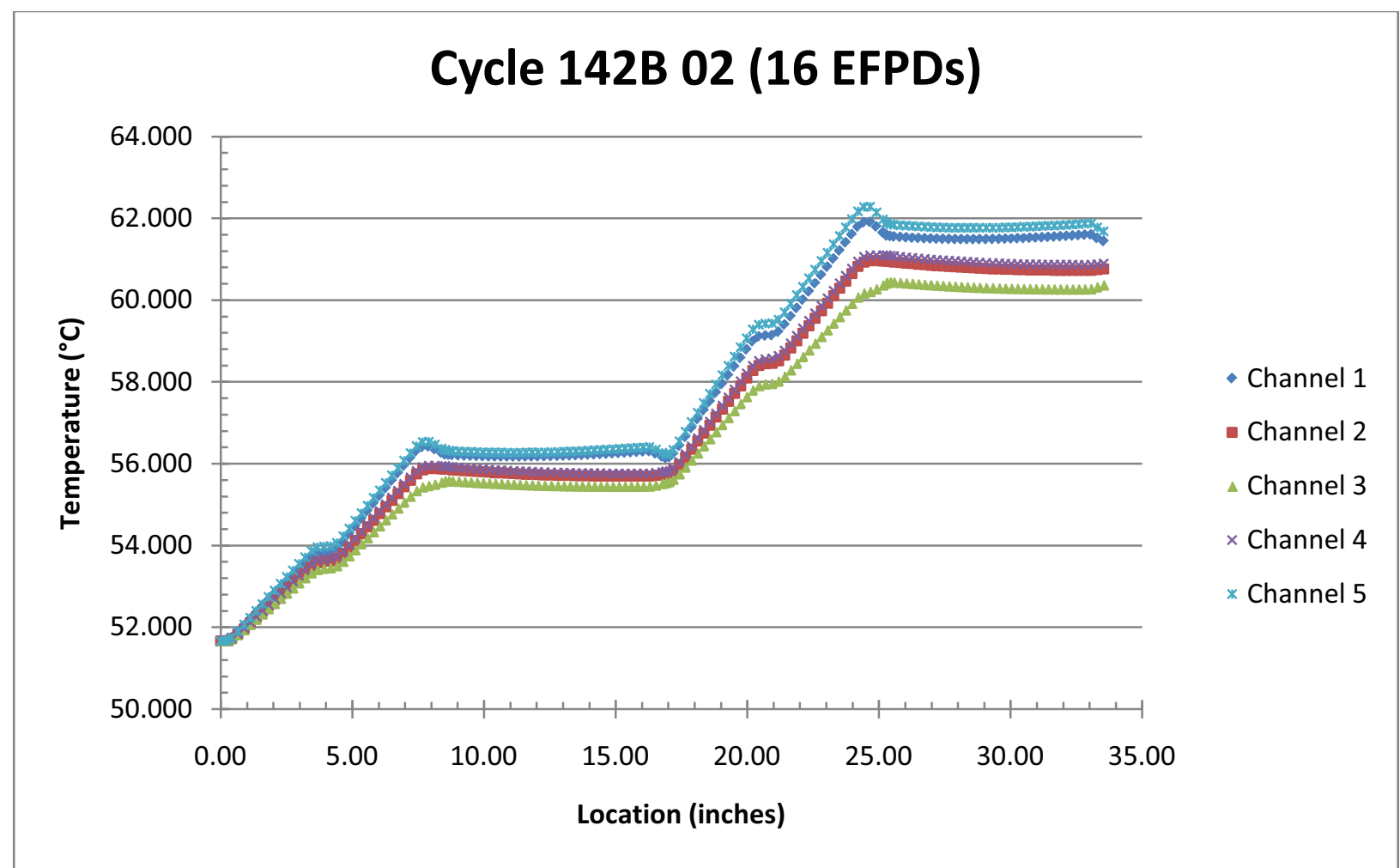

Figure 10. Coolant temperature as a function of location along the test assembly for Cycle 142B 02. ${ }^{9}$

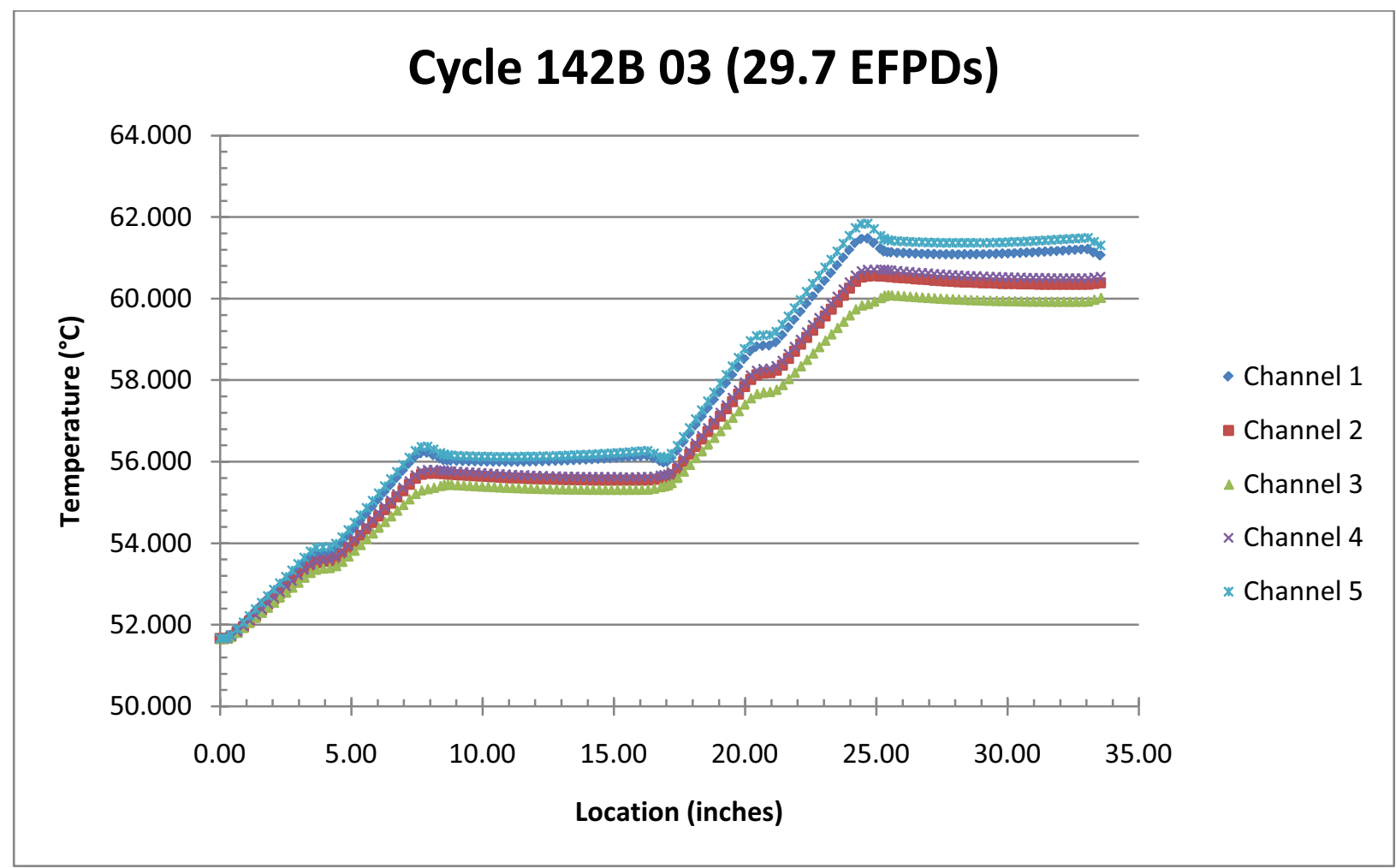

Figure 11. Coolant temperature as a function of location along the test assembly for Cycle 142B 03. ${ }^{9}$ 


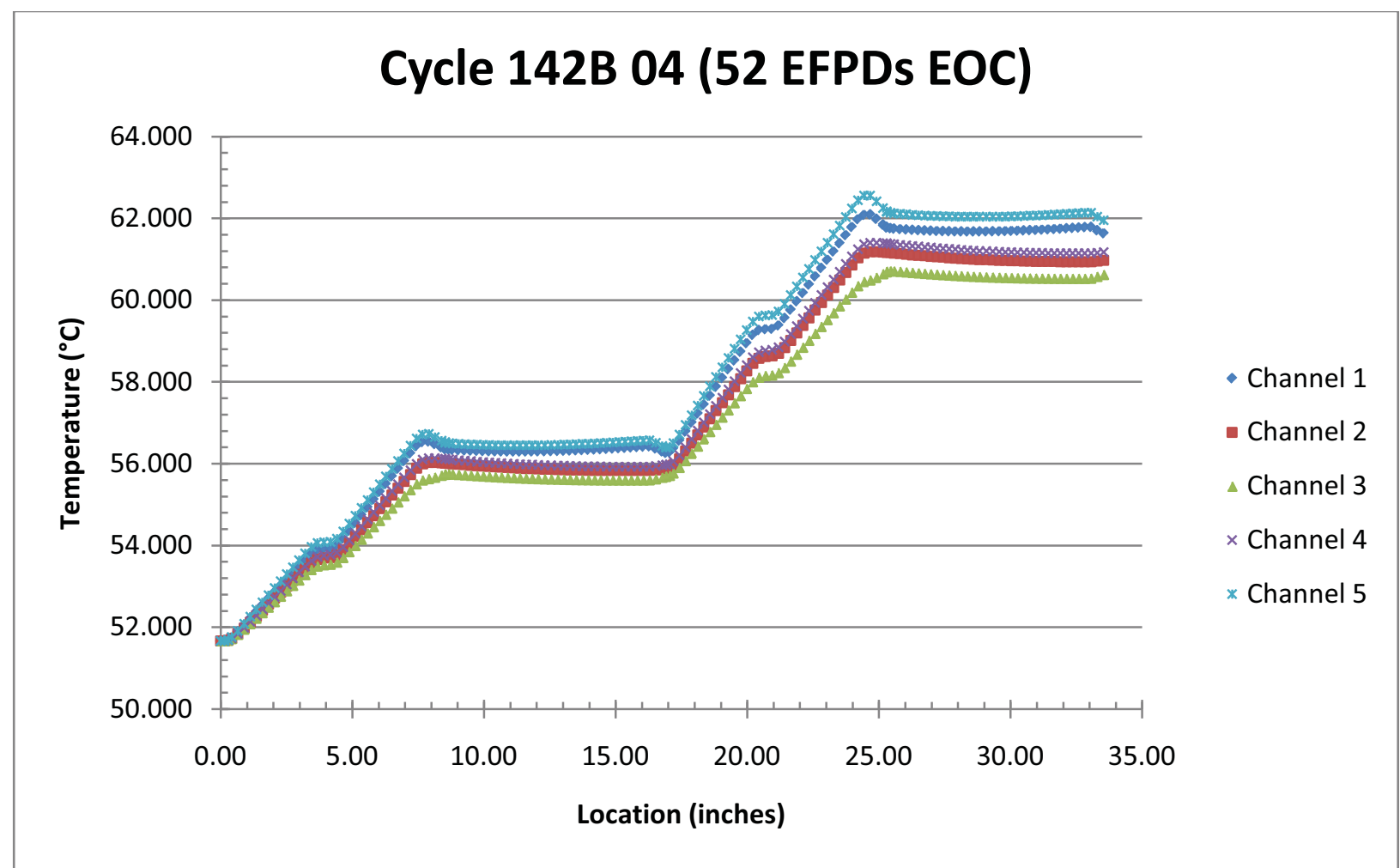

Figure 12. Coolant temperature as a function of location along the test assembly for Cycle 142B 04 (EOC). ${ }^{9}$ 


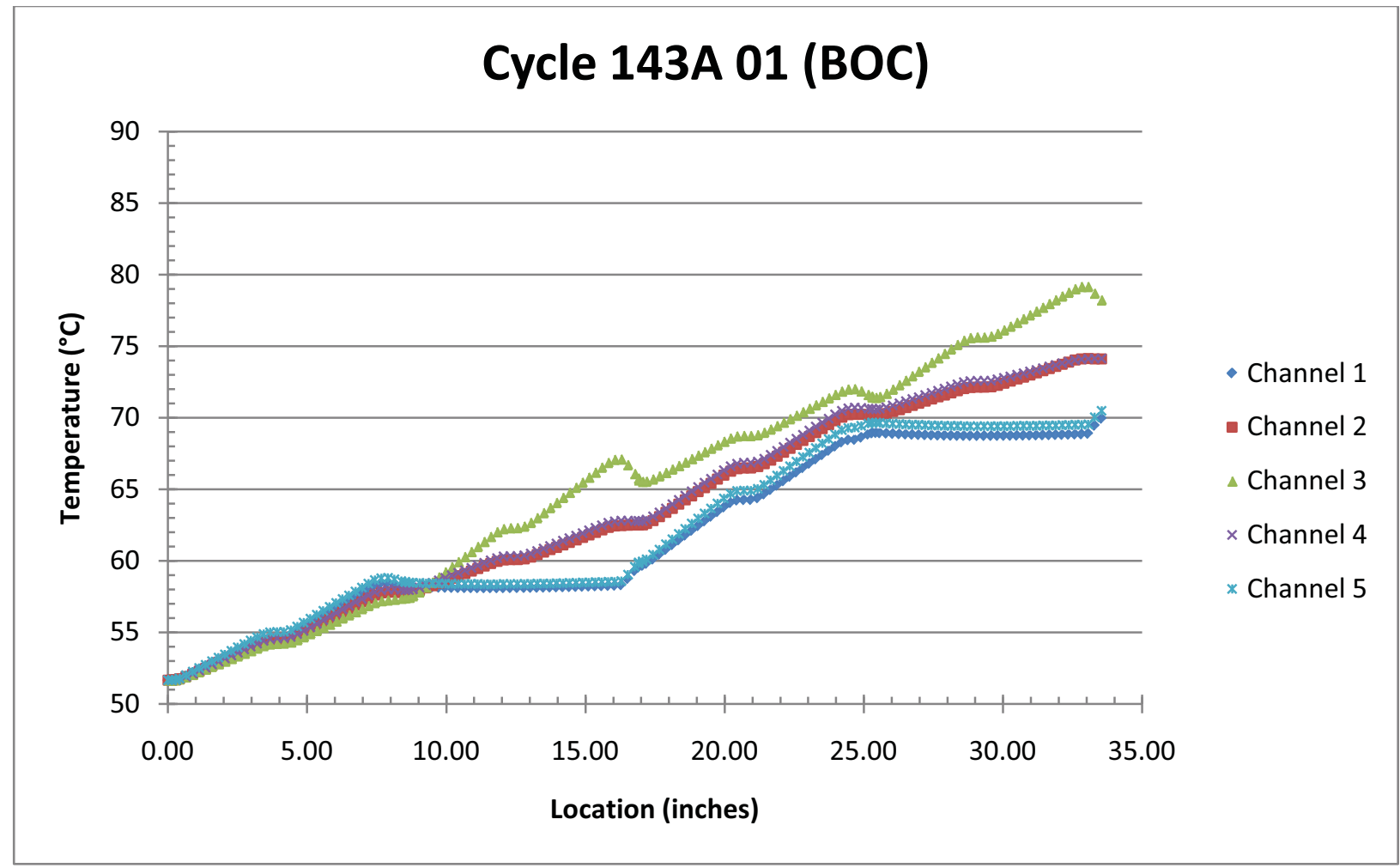

Figure 13. Coolant temperature as a function of location along the test assembly for Cycle 143A 01 (BOC). ${ }^{9}$

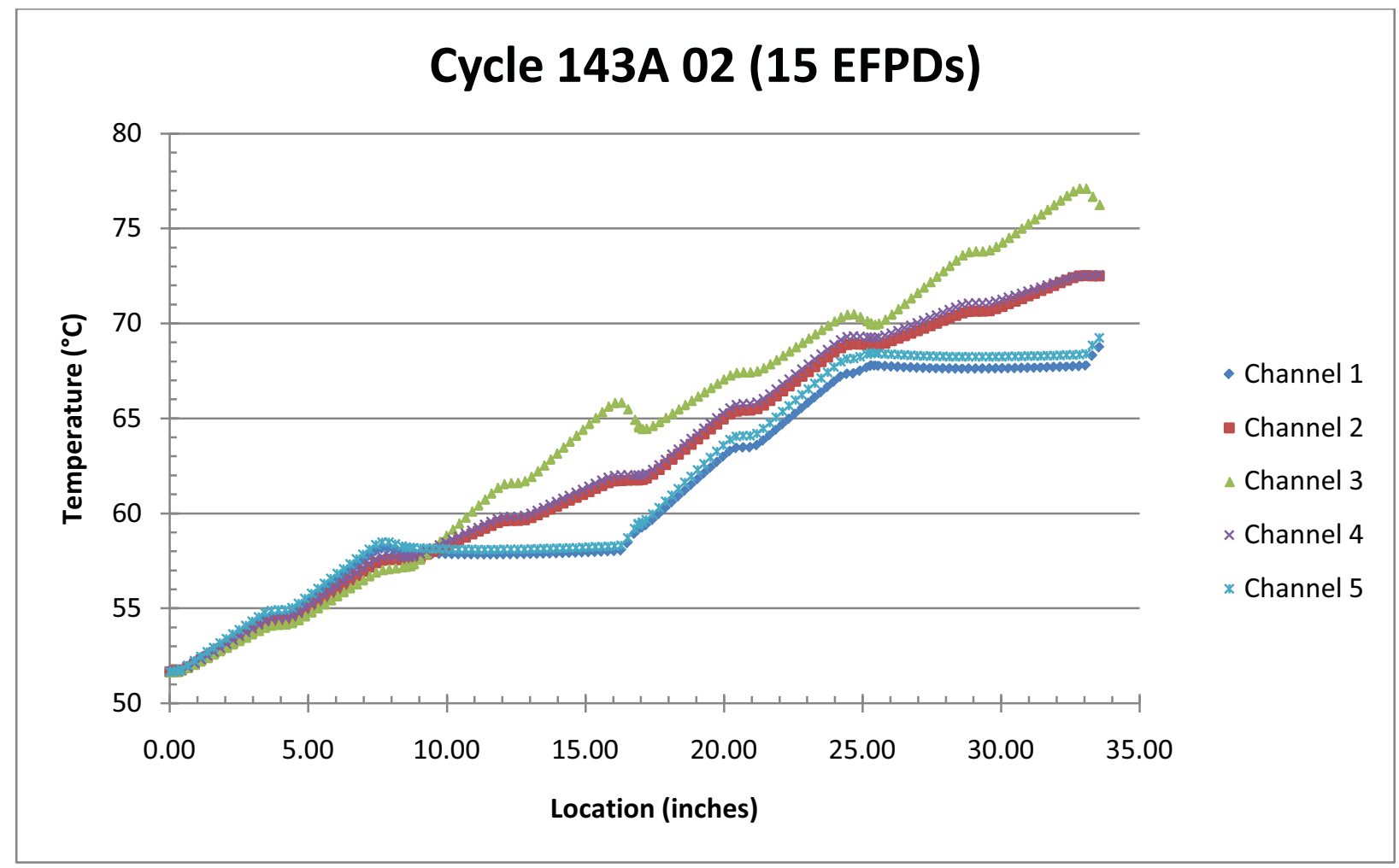

Figure 14. Coolant temperature as a function of location along the test assembly for Cycle 143A 02. ${ }^{9}$ 


\section{Cycle 143A 03 (26 EFPDs)}

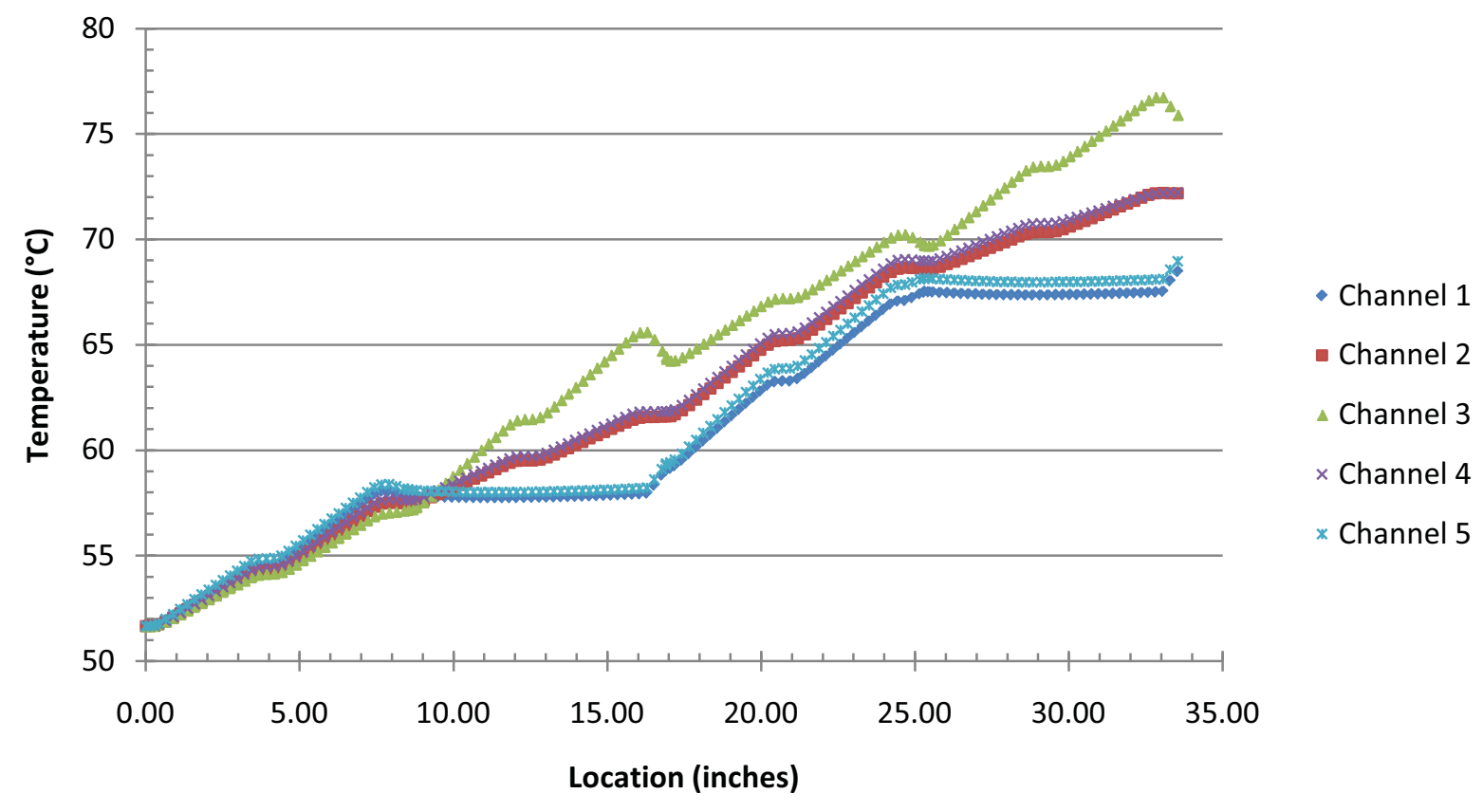

Figure 15. Coolant temperature as a function of location along the test assembly for Cycle 143A 03. ${ }^{9}$

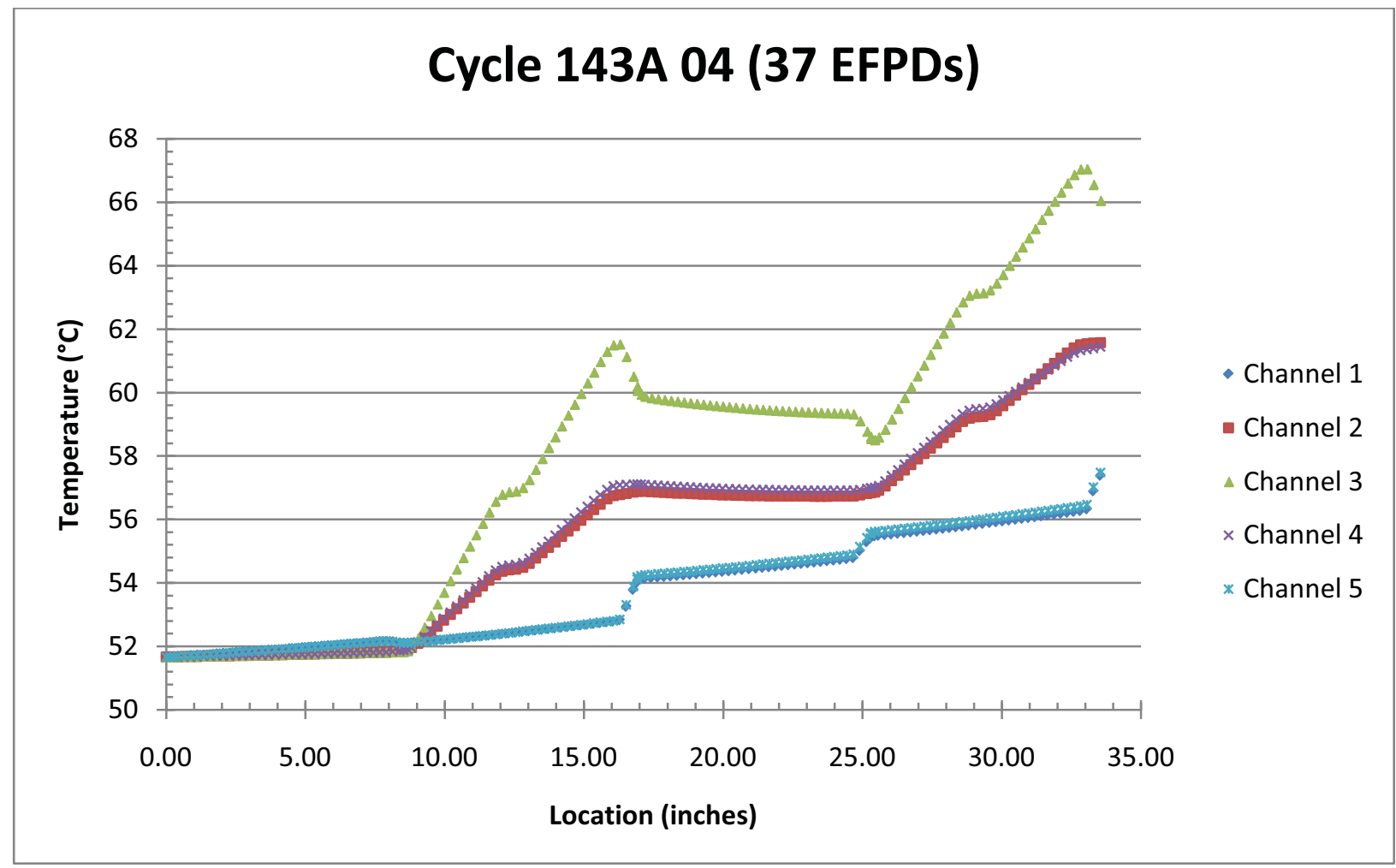

Figure 16. Coolant temperature as a function of location along the test assembly for Cycle 143A 04. ${ }^{9}$ 


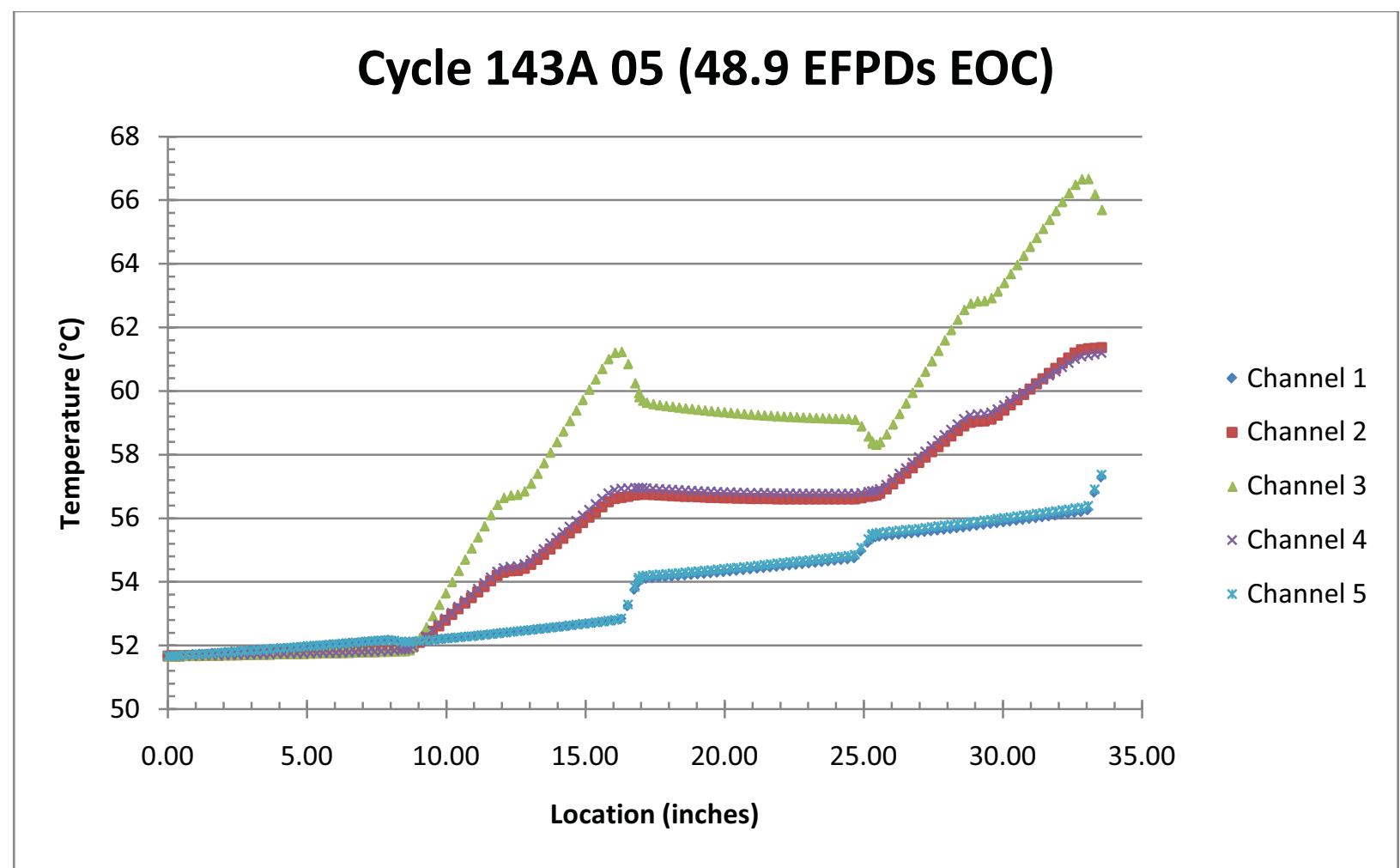

Figure 17. Coolant temperature as a function of location along the test assembly for Cycle 143A 05 (EOC). ${ }^{9}$ 


\subsection{Plate Surface Temperature}

The maximum, minimum, and average plate temperatures for each cycle interval are provided in Table 15 through Table 23.

Table 15. Plate surface temperatures for Cycle 142B 01 (BOC). ${ }^{9}$

\begin{tabular}{|c|c|c|c|c|}
\hline \multirow{2}{*}{$\begin{array}{c}\text { Fuel } \\
\text { Plate }\end{array}$} & \multirow{2}{*}{ Plate ID } & \multicolumn{3}{|c|}{ BOC (0 EFPDs $)$} \\
\cline { 3 - 5 } & & $\begin{array}{c}\text { Max Temp } \\
\left({ }^{\circ} \text { C }\right)\end{array}$ & $\begin{array}{c}\text { Min Temp } \\
\left({ }^{\circ} \text { C }\right)\end{array}$ & $\begin{array}{c}\text { Ave Temp } \\
\left({ }^{\circ} \text { C }\right)\end{array}$ \\
\hline A-1 & L1P30Z & 83.405 & 52.622 & 68.629 \\
\hline A-2 & L1P256 & 77.503 & 52.565 & 65.899 \\
\hline A-3 & L2P15Z & 77.459 & 52.564 & 65.893 \\
\hline A-4 & L1P135 & 84.365 & 52.633 & 70.762 \\
\hline A-5 & L1P234 & 92.528 & 54.632 & 73.599 \\
\hline A-6 & L1P213 & 83.646 & 54.352 & 69.448 \\
\hline A-7 & L1P192 & 84.576 & 54.356 & 69.918 \\
\hline A-8 & L1P171 & 92.096 & 54.695 & 73.415 \\
\hline B-1 & DUMMY & -- & -- & -- \\
\hline B-2 & L1F401 & -- & -- & -- \\
\hline B-3 & L1F417 & -- & -- & -- \\
\hline B-4 & DUMMY & -- & -- & -- \\
\hline B-5 & DUMMY & -- & -- & -- \\
\hline B-6 & L2F46Z & -- & -- & -- \\
\hline B-7 & L1F44N & -- & -- & -- \\
\hline B-8 & DUMMY & -- & -- & -- \\
\hline C-1 & L1P12Z & 100.586 & 57.463 & 79.602 \\
\hline C-2 & L1P266 & 91.158 & 56.928 & 75.169 \\
\hline C-3 & L2P16Z & 90.237 & 56.924 & 74.704 \\
\hline C-4 & L1P145 & 101.672 & 57.553 & 80.148 \\
\hline C-5 & L1P244 & 102.166 & 60.010 & 81.057 \\
\hline C-6 & L1P223 & 92.733 & 59.322 & 76.589 \\
\hline C-7 & L1P202 & 93.184 & 59.308 & 76.792 \\
\hline C-8 & L1P181 & 102.554 & 60.111 & 81.286 \\
\hline D-1 & DUMMY & -- & -- & -- \\
\hline D-2 & L1F381 & -- & -- & -- \\
\hline D-3 & L1F427 & -- & -- & -- \\
\hline D-4 & DUMMY & -- & -- & -- \\
\hline D-5 & DUMMY & -- & -- & -- \\
\hline D-6 & L2F45Z & -- & -- & -- \\
\hline D-7 & L2F47Z & -- & -- & -- \\
\hline D-8 & DUMMY & -- & -- & -- \\
\hline & & & & \\
\hline
\end{tabular}


Table 16. Plate surface temperatures for Cycle 142B 02. ${ }^{9}$

\begin{tabular}{|c|c|c|c|c|}
\hline $\begin{array}{c}\text { Fuel } \\
\text { Plate }\end{array}$ & \multirow{2}{*}{ Plate ID } & $\begin{array}{c}\text { Max Temp } \\
\left({ }^{\circ} \mathrm{C}\right)\end{array}$ & $\begin{array}{c}\text { Min Temp } \\
\left({ }^{\circ} \mathrm{C}\right)\end{array}$ & $\begin{array}{c}\text { Ave Temp } \\
\left({ }^{\circ} \mathrm{C}\right)\end{array}$ \\
\hline A-1 & L1P30Z & 82.565 & 52.609 & 68.176 \\
\hline A-2 & L1P256 & 77.156 & 52.556 & 65.689 \\
\hline A-3 & L2P15Z & 77.189 & 52.558 & 65.733 \\
\hline A-4 & L1P135 & 83.848 & 52.625 & 68.801 \\
\hline A-5 & L1P234 & 91.286 & 54.572 & 72.932 \\
\hline A-6 & L1P213 & 82.273 & 54.318 & 68.723 \\
\hline A-7 & L1P192 & 83.758 & 54.326 & 69.474 \\
\hline A-8 & L1P171 & 90.619 & 54.655 & 72.646 \\
\hline B-1 & DUMMY & -- & -- & -- \\
\hline B-2 & L1F401 & -- & -- & -- \\
\hline B-3 & L1F417 & -- & -- & -- \\
\hline B-4 & DUMMY & -- & -- & -- \\
\hline B-5 & DUMMY & -- & -- & -- \\
\hline B-6 & L2F46Z & -- & -- & -- \\
\hline B-7 & L1F44N & -- & -- & -- \\
\hline B-8 & DUMMY & -- & -- & -- \\
\hline C-1 & L1P12Z & 96.345 & 57.249 & 77.365 \\
\hline C-2 & L1P266 & 88.359 & 56.754 & 73.587 \\
\hline C-3 & L2P16Z & 88.109 & 56.769 & 73.455 \\
\hline C-4 & L1P145 & 98.245 & 57.356 & 78.323 \\
\hline C-5 & L1P244 & 98.487 & 59.576 & 79.006 \\
\hline C-6 & L1P223 & 90.054 & 58.974 & 75.028 \\
\hline C-7 & L1P202 & 89.918 & 58.992 & 74.957 \\
\hline C-8 & L1P181 & 99.299 & 59.747 & 79.474 \\
\hline D-1 & DUMMY & -- & -- & -- \\
\hline D-2 & L1F381 & -- & -- & -- \\
\hline D-3 & L1F427 & -- & -- & -- \\
\hline D-4 & DUMMY & -- & -- & -- \\
\hline D-5 & DUMMY & -- & -- & -- \\
\hline D-6 & L2F45Z & -- & -- & -- \\
\hline D-7 & L2F47Z & -- & -- & - \\
\hline D-8 & DUMMY & -- & - & - \\
\hline
\end{tabular}


Table 17. Plate surface temperatures for Cycle 142B 03. ${ }^{9}$

\begin{tabular}{|c|c|c|c|c|}
\hline $\begin{array}{c}\text { Fuel } \\
\text { Plate }\end{array}$ & Plate ID & $\begin{array}{c}\text { Max Temp } \\
\left({ }^{\circ} \mathrm{C}\right)\end{array}$ & $\begin{array}{c}\text { Min Temp } \\
\left({ }^{\circ} \mathrm{C}\right)\end{array}$ & $\begin{array}{c}\text { Ave Temp } \\
\left({ }^{\circ} \mathrm{C}\right)\end{array}$ \\
\hline A-1 & L1P30Z & 81.611 & 52.590 & 67.653 \\
\hline A-2 & L1P256 & 76.543 & 52.542 & 65.348 \\
\hline A-3 & L2P15Z & 76.480 & 52.542 & 64.228 \\
\hline A-4 & L1P135 & 82.902 & 52.614 & 68.291 \\
\hline A-5 & L1P234 & 89.499 & 54.499 & 71.990 \\
\hline A-6 & L1P213 & 81.354 & 54.256 & 68.216 \\
\hline A-7 & L1P192 & 82.665 & 54.261 & 68.874 \\
\hline A-8 & L1P171 & 89.338 & 54.581 & 71.955 \\
\hline B-1 & DUMMY & -- & -- & -- \\
\hline B-2 & L1F401 & -- & -- & -- \\
\hline B-3 & L1F417 & -- & -- & -- \\
\hline B-4 & DUMMY & -- & -- & -- \\
\hline B-5 & DUMMY & -- & -- & -- \\
\hline B-6 & L2F46Z & -- & -- & -- \\
\hline B-7 & L1F44N & -- & -- & -- \\
\hline B-8 & DUMMY & -- & -- & -- \\
\hline C-1 & L1P12Z & 94.474 & 57.067 & 76.330 \\
\hline C-2 & L1P266 & 86.889 & 56.579 & 72.721 \\
\hline C-3 & L2P16Z & 86.679 & 56.614 & 72.623 \\
\hline C-4 & L1P145 & 95.817 & 57.175 & 77.025 \\
\hline C-5 & L1P244 & 96.193 & 59.300 & 77.741 \\
\hline C-6 & L1P223 & 88.356 & 58.726 & 74.029 \\
\hline C-7 & L1P202 & 88.839 & 58.742 & 74.270 \\
\hline C-8 & L1P181 & 97.278 & 59.449 & 78.329 \\
\hline D-1 & DUMMY & -- & -- & -- \\
\hline D-2 & L1F381 & -- & -- & -- \\
\hline D-3 & L1F427 & -- & -- & -- \\
\hline D-4 & DUMMY & -- & -- & - \\
\hline D-5 & DUMMY & -- & -- & -- \\
\hline D-6 & L2F45Z & -- & -- & - \\
\hline D-7 & L2F47Z & -- & -- & - \\
\hline D-8 & DUMMY & -- & - & - \\
\hline
\end{tabular}


Table 18. Plate surface temperatures for Cycle 142B 04 (EOC). ${ }^{9}$

\begin{tabular}{|c|c|c|c|c|}
\hline \multirow{2}{*}{$\begin{array}{l}\text { Fuel } \\
\text { Plate }\end{array}$} & \multirow[b]{2}{*}{ Plate ID } & \multicolumn{3}{|c|}{ EOC (52 EFPDs) } \\
\hline & & $\begin{array}{c}\text { Max Temp } \\
\left({ }^{\circ} \mathrm{C}\right)\end{array}$ & $\begin{array}{c}\text { Min Temp } \\
\left({ }^{\circ} \mathrm{C}\right)\end{array}$ & $\begin{array}{c}\text { Ave Temp } \\
\left({ }^{\circ} \mathrm{C}\right)\end{array}$ \\
\hline$A-1$ & L1P30Z & 83.490 & 52.626 & 68.670 \\
\hline A-2 & L1P256 & 78.300 & 52.582 & 66.316 \\
\hline$A-3$ & L2P15Z & 78.288 & 52.582 & 66.334 \\
\hline$A-4$ & L1P135 & 85.184 & 52.645 & 69.514 \\
\hline$A-5$ & L1P234 & 92.027 & 54.654 & 73.360 \\
\hline$A-6$ & L1P213 & 83.488 & 54.419 & 69.406 \\
\hline A-7 & L1P192 & 84.905 & 54.430 & 70.121 \\
\hline A-8 & L1P171 & 91.890 & 54.762 & 73.352 \\
\hline B-1 & DUMMY & -- & -- & -- \\
\hline B-2 & L1F401 & -- & -- & -- \\
\hline B-3 & L1F417 & -- & -- & -- \\
\hline B-4 & DUMMY & -- & -- & -- \\
\hline B-5 & DUMMY & -- & -- & -- \\
\hline B-6 & L2F46Z & -- & -- & -- \\
\hline B-7 & L1F44N & -- & -- & -- \\
\hline B-8 & DUMMY & -- & -- & - \\
\hline $\mathrm{C}-1$ & L1P12Z & 96.871 & 57.380 & 77.693 \\
\hline $\mathrm{C}-2$ & L1P266 & 89.076 & 56.906 & 73.487 \\
\hline C-3 & L2P16Z & 88.952 & 56.933 & 73.993 \\
\hline C-4 & L1P145 & 98.824 & 57.520 & 78.701 \\
\hline C-5 & L1P244 & 98.973 & 59.739 & 79.330 \\
\hline C-6 & L1P223 & 90.705 & 59.166 & 75.469 \\
\hline C-7 & L1P202 & 91.439 & 59.194 & 75.840 \\
\hline C-8 & L1P181 & 100.414 & 59.942 & 80.125 \\
\hline$D-1$ & DUMMY & -- & -- & -- \\
\hline $\mathrm{D}-2$ & L1F381 & -- & -- & -- \\
\hline$D-3$ & L1F427 & -- & -- & -- \\
\hline$D-4$ & DUMMY & -- & -- & -- \\
\hline$D-5$ & DUMMY & -- & -- & -- \\
\hline D-6 & L2F45Z & -- & -- & -- \\
\hline D-7 & L2F47Z & -- & -- & -- \\
\hline D-8 & DUMMY & -- & -- & -- \\
\hline
\end{tabular}


Table 19. Plate surface temperature for Cycle 143A 01 (BOC). ${ }^{9}$

\begin{tabular}{|c|c|c|c|c|}
\hline \multirow{2}{*}{$\begin{array}{c}\text { Fuel } \\
\text { Plate }\end{array}$} & \multirow{2}{*}{ Plate ID } & $\begin{array}{c}\text { Max Temp } \\
\left({ }^{\circ} \mathrm{C}\right)\end{array}$ & $\begin{array}{c}\text { Min Temp } \\
\left({ }^{\circ} \mathrm{C}\right)\end{array}$ & $\begin{array}{c}\text { Ave Temp } \\
\left({ }^{\circ} \mathrm{C}\right)\end{array}$ \\
\hline A-1 & L1P30Z & 93.458 & 52.858 & 74.176 \\
\hline A-2 & L1P256 & 86.752 & 52.828 & 71.090 \\
\hline A-3 & L2P15Z & 87.105 & 52.837 & 71.308 \\
\hline A-4 & L1P135 & 96.589 & 52.896 & 75.769 \\
\hline A-5 & L1P234 & 106.825 & 55.586 & 81.275 \\
\hline A-6 & L1P213 & 94.900 & 55.288 & 75.867 \\
\hline A-7 & L1P192 & 96.982 & 55.323 & 60.247 \\
\hline A-8 & L1P171 & 106.747 & 55.801 & 81.352 \\
\hline B-1 & DUMMY & -- & -- & -- \\
\hline B-2 & L1F401 & 119.004 & 58.296 & 90.275 \\
\hline B-3 & L1F417 & 120.060 & 58.372 & 90.937 \\
\hline B-4 & DUMMY & -- & -- & -- \\
\hline B-5 & DUMMY & -- & -- & -- \\
\hline B-6 & L2F46Z & 123.609 & 61.466 & 93.078 \\
\hline B-7 & L1F44N & 125.757 & 61.603 & 94.296 \\
\hline B-8 & DUMMY & -- & -- & -- \\
\hline C-1 & L1P12Z & 118.990 & 61.758 & 91.723 \\
\hline C-2 & L1P266 & 111.233 & 63.856 & 88.622 \\
\hline C-3 & L2P16Z & 110.584 & 63.995 & 88.407 \\
\hline C-4 & L1P145 & 122.179 & 62.063 & 93.457 \\
\hline C-5 & L1P244 & 120.921 & 65.654 & 93.790 \\
\hline C-6 & L1P223 & 113.261 & 67.060 & 90.773 \\
\hline C-7 & L1P202 & 113.911 & 67.261 & 91.227 \\
\hline C-8 & L1P181 & 123.170 & 66.181 & 95.108 \\
\hline D-1 & DUMMY & -- & -- & -- \\
\hline D-2 & L1F381 & 125.149 & 70.121 & 98.685 \\
\hline D-3 & L1F427 & 125.579 & 70.330 & 98.876 \\
\hline D-4 & DUMMY & -- & -- & -- \\
\hline D-5 & DUMMY & -- & -- & -- \\
\hline D-6 & L2F45Z & 126.891 & 72.594 & 100.162 \\
\hline D-7 & L2F47Z & 118.089 & 72.820 & 95.762 \\
\hline D-8 & DUMMY & -- & -- & -- \\
\hline
\end{tabular}


Table 20. Plate surface temperature for Cycle 143A 02. ${ }^{9}$

\begin{tabular}{|c|c|c|c|c|}
\hline $\begin{array}{c}\text { Fuel } \\
\text { Plate }\end{array}$ & \multirow{2}{*}{ Plate ID } & $\begin{array}{c}\text { Max Temp } \\
\left({ }^{\circ} \mathrm{C}\right)\end{array}$ & $\begin{array}{c}\text { Min Temp } \\
\left({ }^{\circ} \mathrm{C}\right)\end{array}$ & $\begin{array}{c}\text { Ave Temp } \\
\left({ }^{\circ} \mathrm{C}\right)\end{array}$ \\
\hline A-1 & L1P30Z & 91.888 & 52.834 & 73.318 \\
\hline A-2 & L1P256 & 85.793 & 52.806 & 70.559 \\
\hline A-3 & L2P15Z & 86.295 & 52.817 & 70.844 \\
\hline A-4 & L1P135 & 95.192 & 52.875 & 74.992 \\
\hline A-5 & L1P234 & 104.549 & 55.462 & 80.076 \\
\hline A-6 & L1P213 & 93.889 & 55.196 & 75.283 \\
\hline A-7 & L1P192 & 95.504 & 55.238 & 76.122 \\
\hline A-8 & L1P171 & 104.169 & 55.133 & 80.009 \\
\hline B-1 & DUMMY & -- & -- & -- \\
\hline B-2 & L1F401 & 113.760 & 58.034 & 87.323 \\
\hline B-3 & L1F417 & 114.635 & 58.103 & 87.875 \\
\hline B-4 & DUMMY & -- & -- & -- \\
\hline B-5 & DUMMY & -- & -- & -- \\
\hline B-6 & L2F46Z & 117.104 & 60.961 & 89.457 \\
\hline B-7 & L1F44N & 118.863 & 61.109 & 90.455 \\
\hline B-8 & DUMMY & -- & -- & -- \\
\hline C-1 & L1P12Z & 114.215 & 61.245 & 88.935 \\
\hline C-2 & L1P266 & 107.378 & 63.092 & 86.242 \\
\hline C-3 & L2P16Z & 106.764 & 63.214 & 86.036 \\
\hline C-4 & L1P145 & 117.503 & 61.528 & 90.694 \\
\hline C-5 & L1P244 & 116.374 & 64.830 & 91.014 \\
\hline C-6 & L1P223 & 109.408 & 66.061 & 88.298 \\
\hline C-7 & L1P202 & 110.029 & 66.243 & 88.724 \\
\hline C-8 & L1P181 & 118.327 & 65.333 & 92.171 \\
\hline D-1 & DUMMY & -- & -- & -- \\
\hline D-2 & L1F381 & 119.681 & 69.012 & 95.237 \\
\hline D-3 & L1F427 & 120.617 & 69.207 & 95.695 \\
\hline D-4 & DUMMY & -- & -- & -- \\
\hline D-5 & DUMMY & -- & -- & -- \\
\hline D-6 & L2F45Z & 122.305 & 71.270 & 97.095 \\
\hline D-7 & L2F47Z & 114.111 & 71.500 & 93.030 \\
\hline D-8 & DUMMY & -- & -- & -- \\
\hline
\end{tabular}


Table 21. Plate surface temperature for Cycle 143A 03. ${ }^{9}$

\begin{tabular}{|c|c|c|c|c|}
\hline \multirow{2}{*}{$\begin{array}{c}\text { Fuel } \\
\text { Plate }\end{array}$} & \multirow{2}{*}{ Plate ID } & \multicolumn{3}{|c|}{26 EFPDs } \\
\cline { 3 - 5 } & & $\begin{array}{c}\text { Max Temp } \\
\left({ }^{\circ} \mathrm{C}\right)\end{array}$ & $\begin{array}{c}\text { Min Temp } \\
\left({ }^{\circ} \mathrm{C}\right)\end{array}$ & $\begin{array}{c}\text { Ave Temp } \\
\left({ }^{\circ} \mathrm{C}\right)\end{array}$ \\
\hline A-1 & L1P30Z & 91.433 & 52.828 & 73.069 \\
\hline A-2 & L1P256 & 85.619 & 52.801 & 70.459 \\
\hline A-3 & L2P15Z & 86.058 & 52.812 & 70.712 \\
\hline A-4 & L1P135 & 94.663 & 52.867 & 74.703 \\
\hline A-5 & L1P234 & 103.779 & 55.428 & 79.676 \\
\hline A-6 & L1P213 & 93.598 & 55.176 & 75.120 \\
\hline A-7 & L1P192 & 95.237 & 55.215 & 75.970 \\
\hline A-8 & L1P171 & 103.409 & 55.648 & 79.612 \\
\hline B-1 & DUMMY & -- & -- & -- \\
\hline B-2 & L1F401 & 112.683 & 57.972 & 86.714 \\
\hline B-3 & L1F417 & 113.546 & 58.039 & 87.256 \\
\hline B-4 & DUMMY & -- & -- & -- \\
\hline B-5 & DUMMY & -- & -- & -- \\
\hline B-6 & L2F46Z & 115.899 & 60.838 & 88.776 \\
\hline B-7 & L1F44N & 117.546 & 60.982 & 89.715 \\
\hline B-8 & DUMMY & -- & -- & -- \\
\hline C-1 & L1P12Z & 113.166 & 61.124 & 88.324 \\
\hline C-2 & L1P266 & 106.838 & 62.935 & 85.848 \\
\hline C-3 & L2P16Z & 106.210 & 63.052 & 85.668 \\
\hline C-4 & L1P145 & 116.275 & 61.397 & 89.988 \\
\hline C-5 & L1P244 & 115.299 & 64.646 & 90.376 \\
\hline C-6 & L1P223 & 108.888 & 65.864 & 87.931 \\
\hline C-7 & L1P202 & 109.515 & 66.037 & 88.354 \\
\hline C-8 & L1P181 & 117.143 & 65.128 & 91.468 \\
\hline D-1 & DUMMY & -- & -- & -- \\
\hline D-2 & L1F381 & 118.649 & 68.792 & 94.583 \\
\hline D-3 & L1F427 & 119.529 & 68.978 & 95.003 \\
\hline D-4 & DUMMY & -- & -- & -- \\
\hline D-5 & DUMMY & -- & -- & -- \\
\hline D-6 & L2F45Z & 121.544 & 71.007 & 96.566 \\
\hline D-7 & L2F47Z & 113.205 & 71.227 & 92.420 \\
\hline D-8 & DUMMY & -- & -- & -- \\
\hline & & & & \\
\hline
\end{tabular}


Table 22. Plate surface temperature for Cycle 143A $04 .{ }^{9}$

\begin{tabular}{|c|c|c|c|c|}
\hline $\begin{array}{c}\text { Fuel } \\
\text { Plate }\end{array}$ & \multirow{2}{*}{ Plate ID } & $\begin{array}{c}\text { Max Temp } \\
\left({ }^{\circ} \mathrm{C}\right)\end{array}$ & $\begin{array}{c}\text { Min Temp } \\
\left({ }^{\circ} \mathrm{C}\right)\end{array}$ & $\begin{array}{c}\text { Ave Temp } \\
\left({ }^{\circ} \mathrm{C}\right)\end{array}$ \\
\hline A-1 & L1P30Z & -- & -- & -- \\
\hline A-2 & L1P256 & -- & -- & -- \\
\hline A-3 & L2P15Z & -- & -- & -- \\
\hline A-4 & L1P135 & -- & -- & -- \\
\hline A-5 & L1P234 & -- & -- & -- \\
\hline A-6 & L1P213 & -- & -- & -- \\
\hline A-7 & L1P192 & -- & -- & -- \\
\hline A-8 & L1P171 & -- & -- & -- \\
\hline B-1 & DUMMY & -- & -- & -- \\
\hline B-2 & L1F401 & 112.952 & 53.901 & 58.304 \\
\hline B-3 & L1F417 & 115.854 & 53.971 & 58.388 \\
\hline B-4 & DUMMY & -- & -- & -- \\
\hline B-5 & DUMMY & -- & -- & - \\
\hline B-6 & L2F46Z & 116.010 & 56.680 & 58.443 \\
\hline B-7 & L1F44N & 119.376 & 56.828 & 58.524 \\
\hline B-8 & DUMMY & -- & -- & -- \\
\hline C-1 & L1P12Z & -- & -- & -- \\
\hline C-2 & L1P266 & -- & -- & -- \\
\hline C-3 & L2P16Z & -- & -- & -- \\
\hline C-4 & L1P145 & -- & -- & -- \\
\hline C-5 & L1P244 & -- & -- & -- \\
\hline C-6 & L1P223 & -- & -- & -- \\
\hline C-7 & L1P202 & -- & -- & -- \\
\hline C-8 & L1P181 & -- & -- & -- \\
\hline D-1 & DUMMY & -- & -- & -- \\
\hline D-2 & L1F381 & 115.055 & 59.255 & 88.372 \\
\hline D-3 & L1F427 & 117.186 & 59.391 & 89.368 \\
\hline D-4 & DUMMY & -- & -- & -- \\
\hline D-5 & DUMMY & -- & -- & -- \\
\hline D-6 & L2F45Z & 119.067 & 61.601 & 90.746 \\
\hline D-7 & L2F47Z & 110.632 & 61.571 & 86.494 \\
\hline D-8 & DUMMY & -- & -- & -- \\
\hline
\end{tabular}


Table 23. Plate surface temperature for Cycle 143B 05 (EOC). ${ }^{9}$

\begin{tabular}{|c|c|c|c|c|}
\hline \multirow{2}{*}{$\begin{array}{c}\text { Fuel } \\
\text { Plate }\end{array}$} & \multirow{2}{*}{ Plate ID } & \multicolumn{3}{|c|}{ EOC $(48.9$ EFPDs $)$} \\
\cline { 3 - 5 } & & $\begin{array}{c}\text { Max Temp } \\
\left.{ }^{\circ} \mathrm{C}\right)\end{array}$ & $\begin{array}{c}\text { Min Temp } \\
\left({ }^{\circ} \mathrm{C}\right)\end{array}$ & $\begin{array}{c}\text { Ave Temp } \\
\left({ }^{\circ} \mathrm{C}\right)\end{array}$ \\
\hline A-1 & L1P30Z & -- & -- & -- \\
\hline A-2 & L1P256 & -- & -- & -- \\
\hline A-3 & L2P15Z & -- & -- & -- \\
\hline A-4 & L1P135 & -- & -- & -- \\
\hline A-5 & L1P234 & -- & -- & -- \\
\hline A-6 & L1P213 & -- & -- & -- \\
\hline A-7 & L1P192 & -- & -- & -- \\
\hline A-8 & L1P171 & -- & -- & -- \\
\hline B-1 & DUMMY & -- & -- & -- \\
\hline B-2 & L1F401 & 111.551 & 53.866 & 58.145 \\
\hline B-3 & L1F417 & 114.186 & 53.929 & 58.223 \\
\hline B-4 & DUMMY & -- & -- & -- \\
\hline B-5 & DUMMY & -- & -- & -- \\
\hline B-6 & L2F46Z & 114.382 & 56.578 & 58.284 \\
\hline B-7 & L1F44N & 117.503 & 56.712 & 58.359 \\
\hline B-8 & DUMMY & -- & -- & -- \\
\hline C-1 & L1P12Z & -- & -- & -- \\
\hline C-2 & L1P266 & -- & -- & -- \\
\hline C-3 & L2P16Z & -- & -- & -- \\
\hline C-4 & L1P145 & -- & -- & -- \\
\hline C-5 & L1P244 & -- & -- & -- \\
\hline C-6 & L1P223 & -- & -- & -- \\
\hline C-7 & L1P202 & -- & -- & -- \\
\hline C-8 & L1P181 & -- & -- & -- \\
\hline D-1 & DUMMY & -- & -- & -- \\
\hline D-2 & L1F381 & 113.755 & 59.087 & 87.622 \\
\hline D-3 & L1F427 & 115.666 & 59.210 & 88.492 \\
\hline D-4 & DUMMY & -- & -- & -- \\
\hline D-5 & DUMMY & -- & -- & -- \\
\hline D-6 & L2F45Z & 118.064 & 61.389 & 90.133 \\
\hline D-7 & L2F47Z & 109.369 & 61.349 & 85.738 \\
\hline D-8 & DUMMY & -- & -- & -- \\
\hline
\end{tabular}




\section{REFERENCES}

1. Wachs, D. M, "RERTR Fuel Development and Qualification Plan," INL/EXT-05-0101, August 2009.

2. RERTR Project Personnel, "RERTR-10A Irradiation Experiment in the Advanced Test Reactor: As-Built Data Package" RERTR-10A, June 2008.

3. RERTR Project Personnel, "RERTR-10B Irradiation Experiment in the Advanced Test Reactor: As-Built Data Package," RERTR-10B, September 2008.

4. N. E. Woolstenhulme to D. M. Perez, Interoffice Memorandum "Description of RERTR Test Train" N. E. Woolstenhulme Letter File NEW-04-10.

5. J. R. Mitchell, G. S. Chang, "As-Run Neutronics Analysis of the RERTR-10 Capsules in the ATR B9 and B-11 Positions," ECAR-856, February 2010.

6. J.R. Mitchell, G.S. Chang, "MCNP-Calculated Gradients across RERTR-10 Mini-Plates Irradiated in ATR" ECAR-857, December 2009.

7. D.M. Wachs, "RERTR-Large-B Position Irradiation Vehicle Flow Test” EDF-8292, July 2007.

8. Wachs, D. M., 2007, “Thermal Analysis of the RERTR-9B Irradiation Test,” EDF-8083, July 2007.

9. Roth, G. A., "RERTR-10 As-Run Data,” to D. M. Perez, April 2010 (see Appendix B). 


\section{Appendix A}

\section{Individual Plate Power and Fission Density Plots}




\section{Appendix A \\ Individual Plate Power and Fission Density Plots}

A-1. RERTR-10A

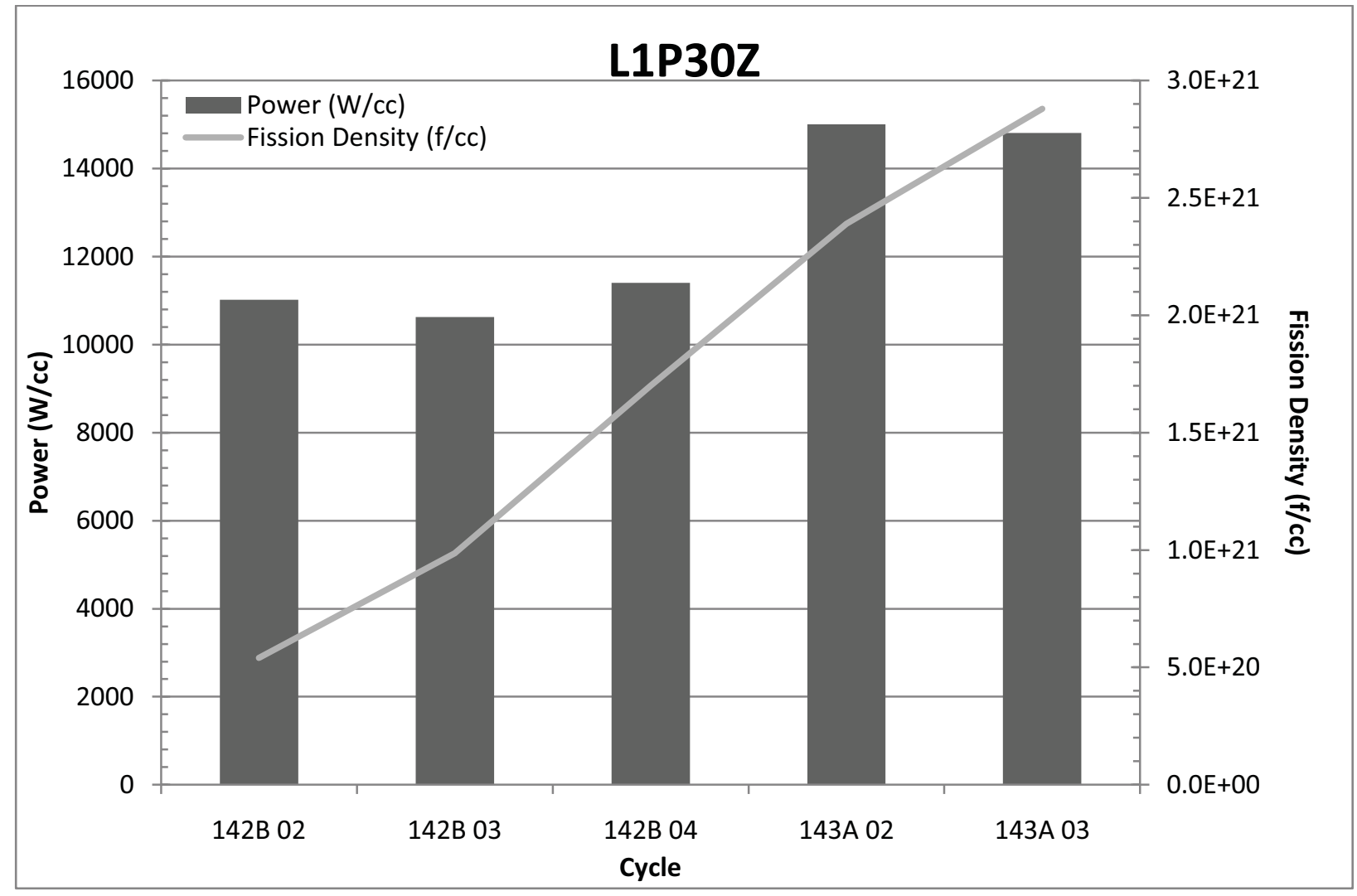



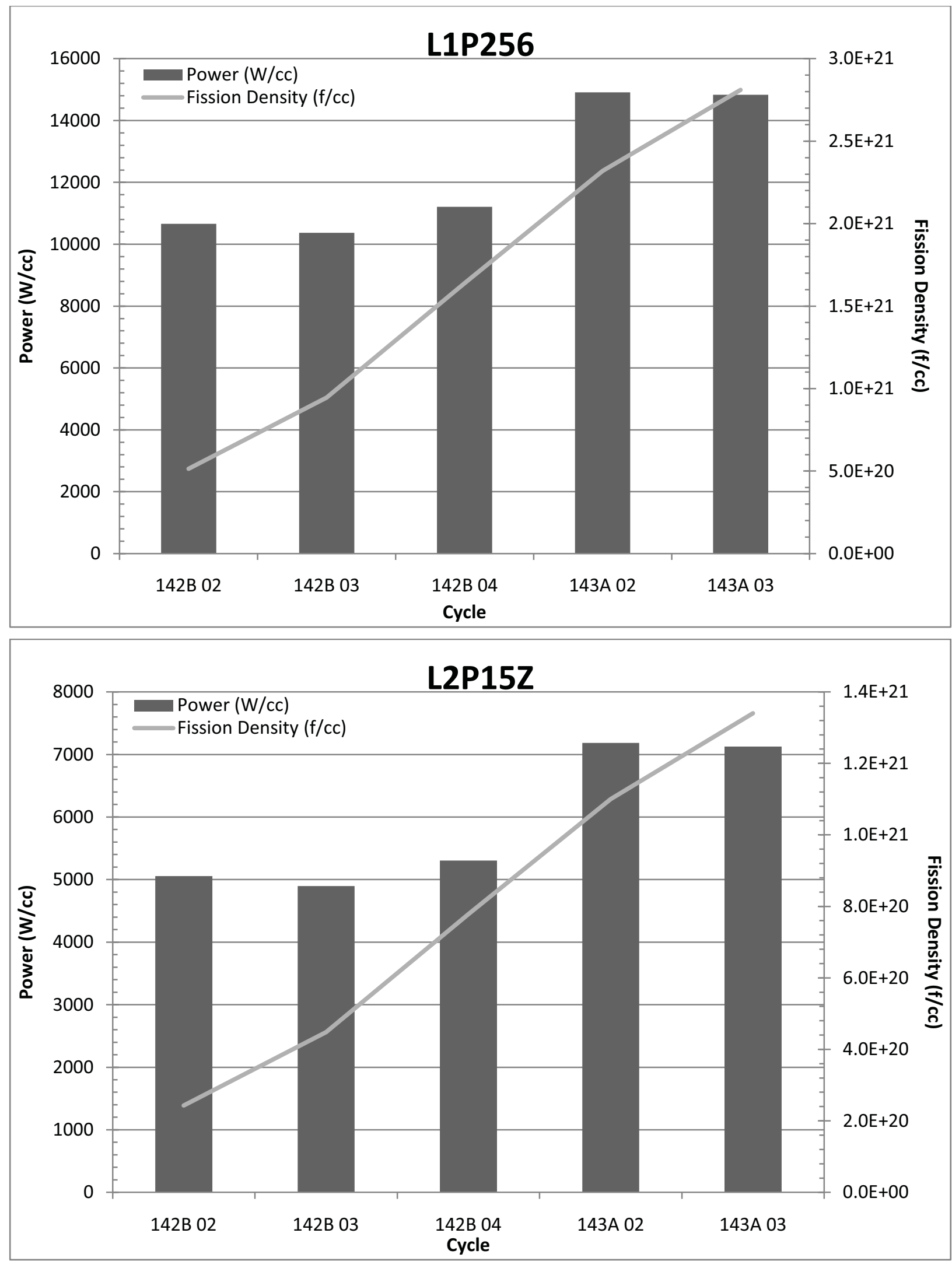

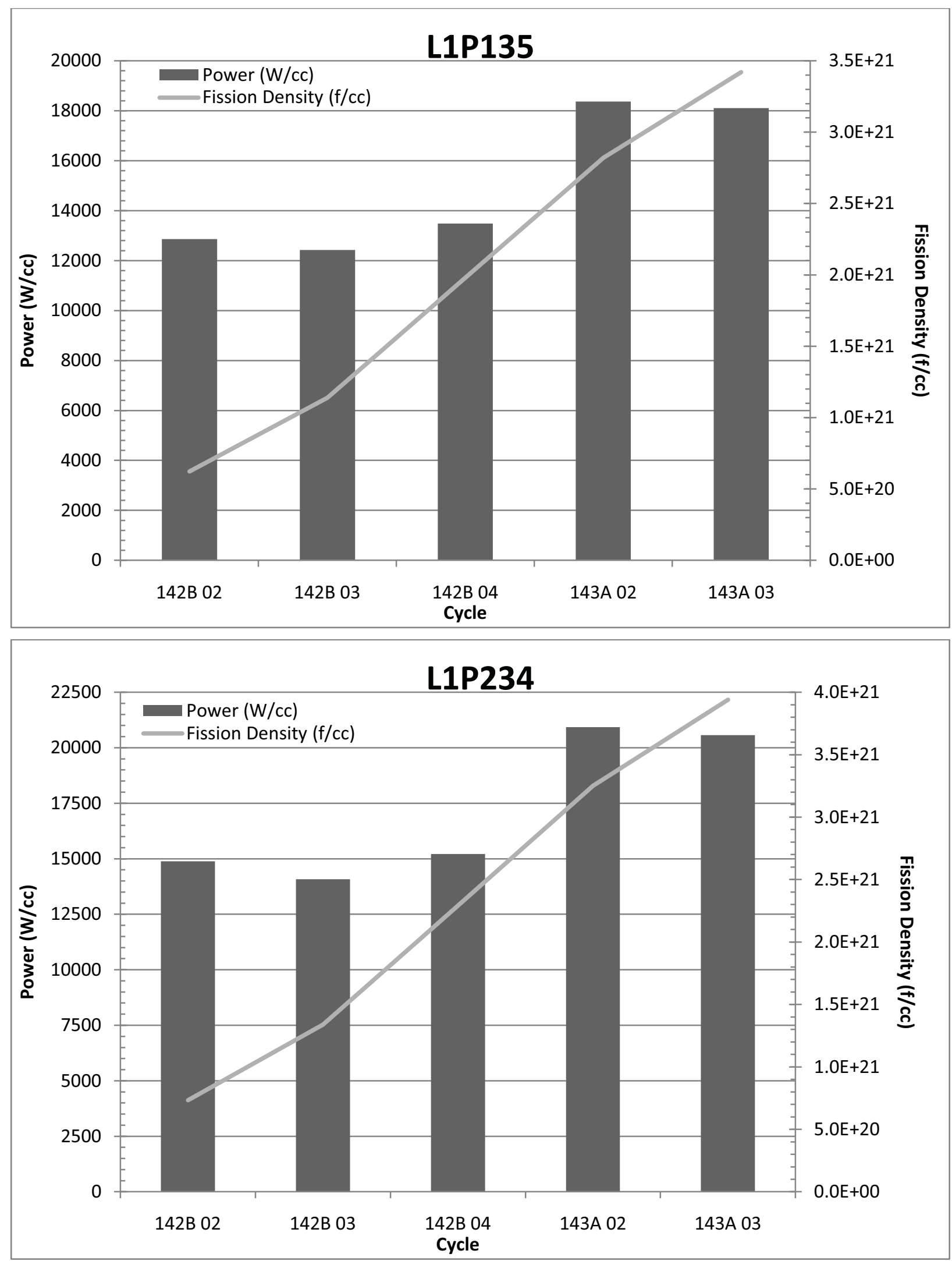

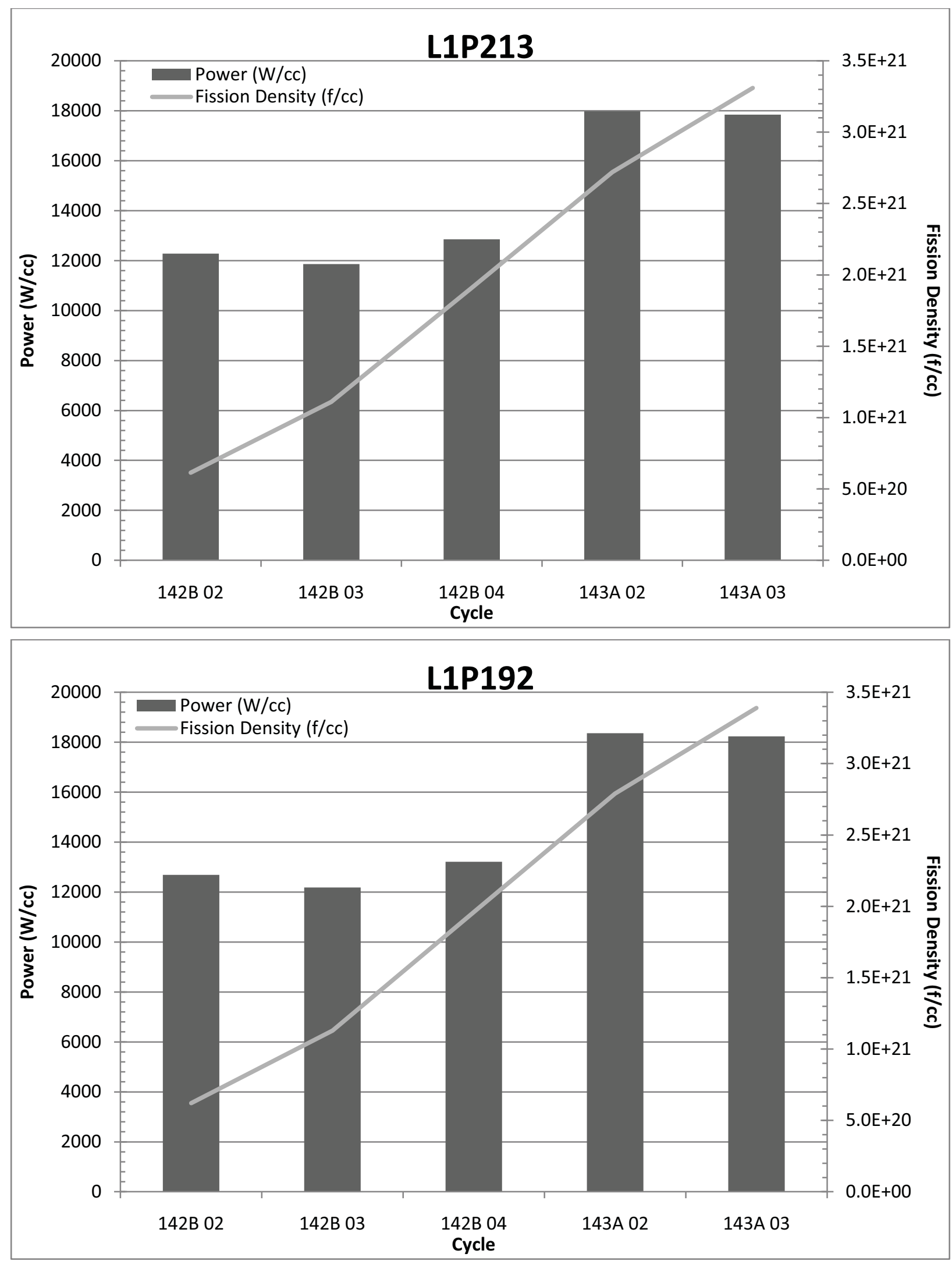

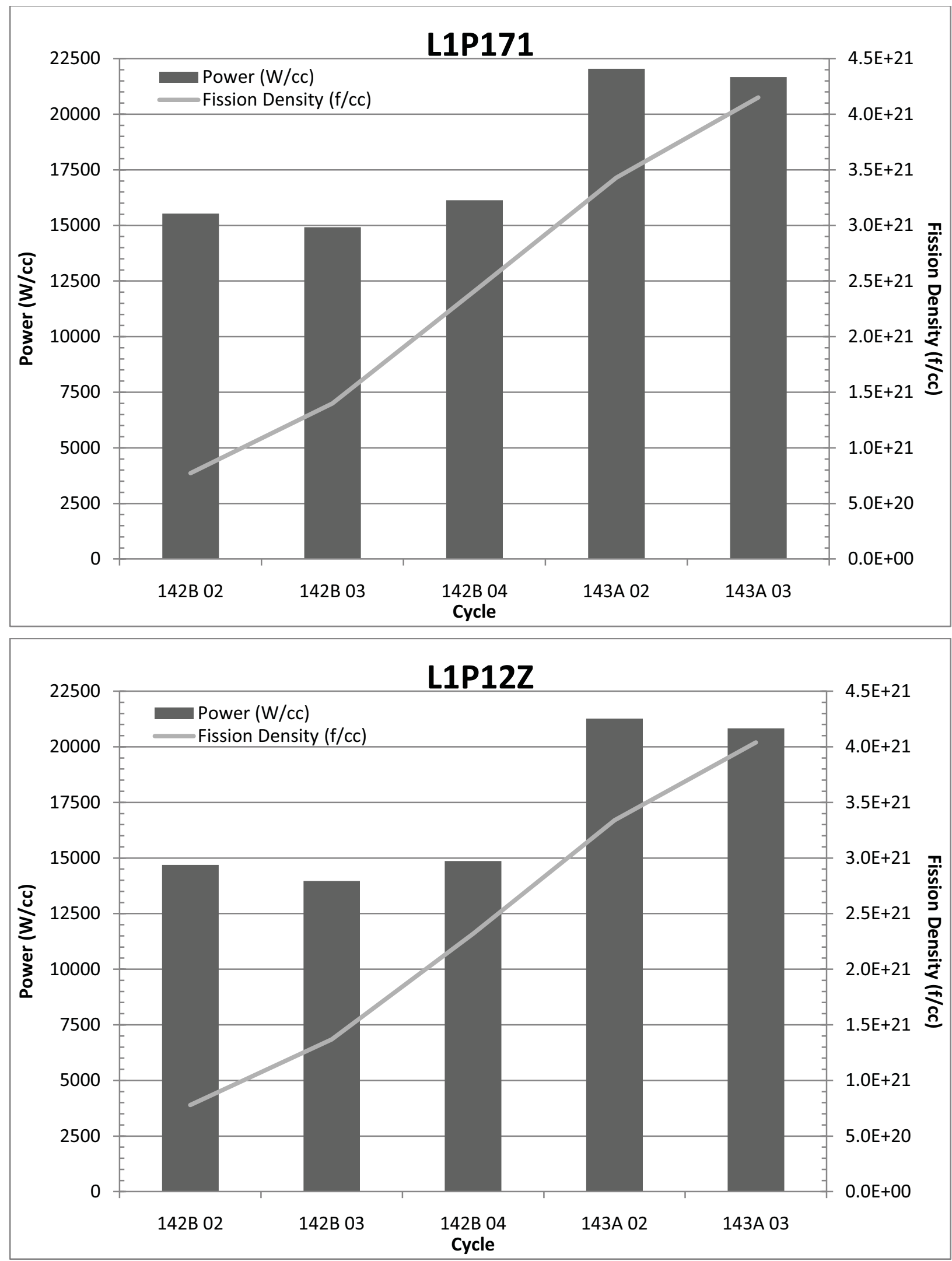

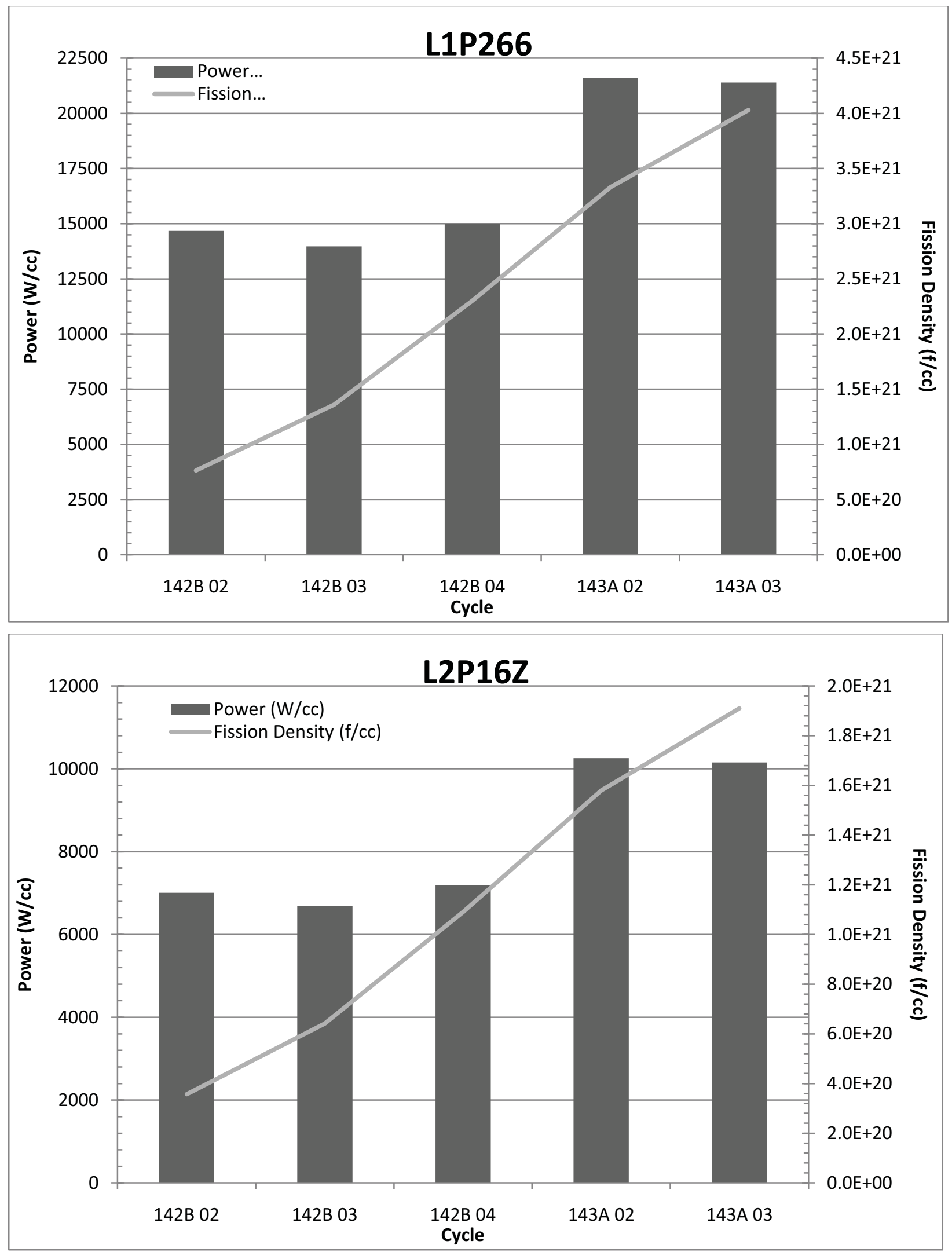

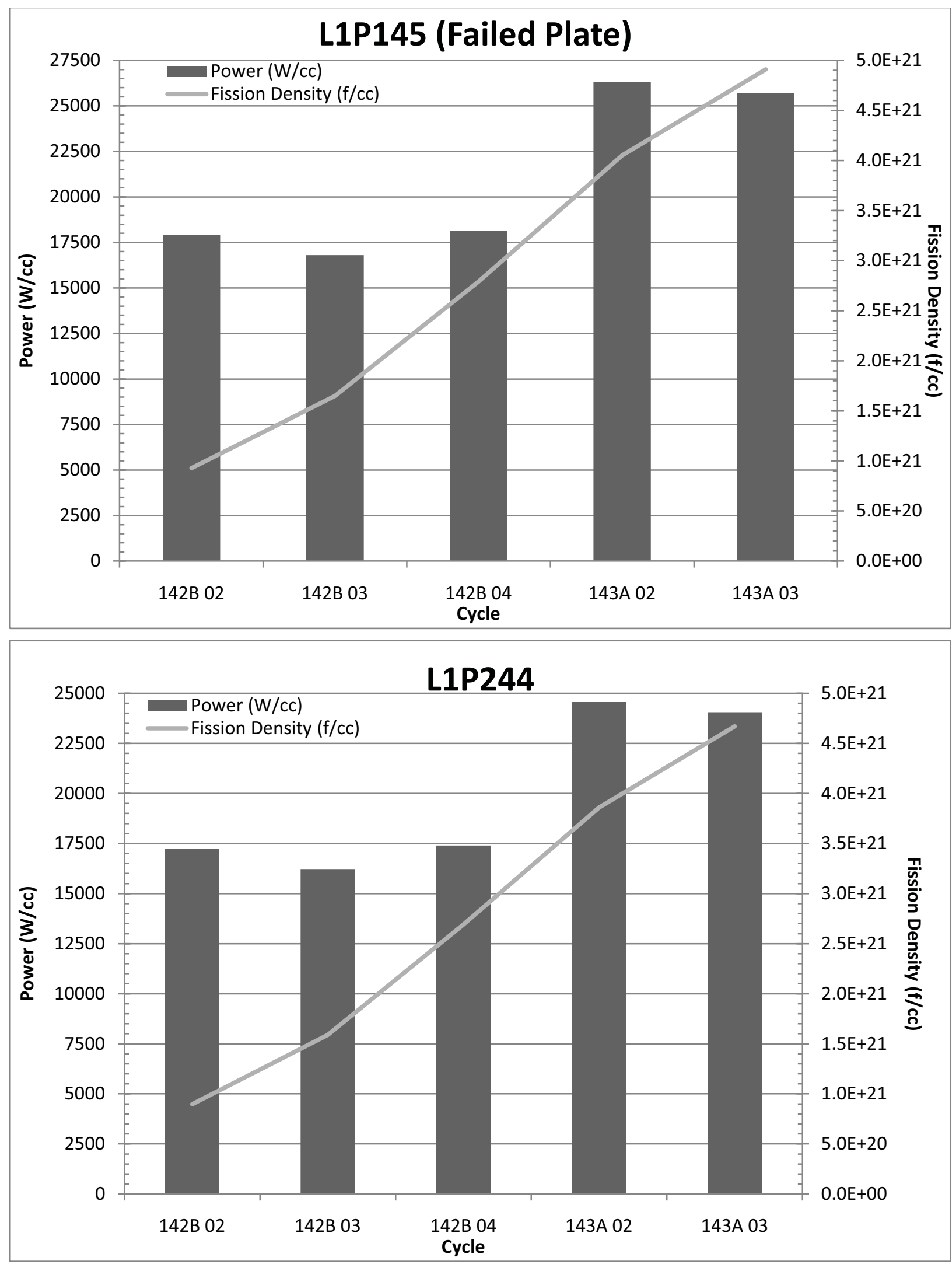

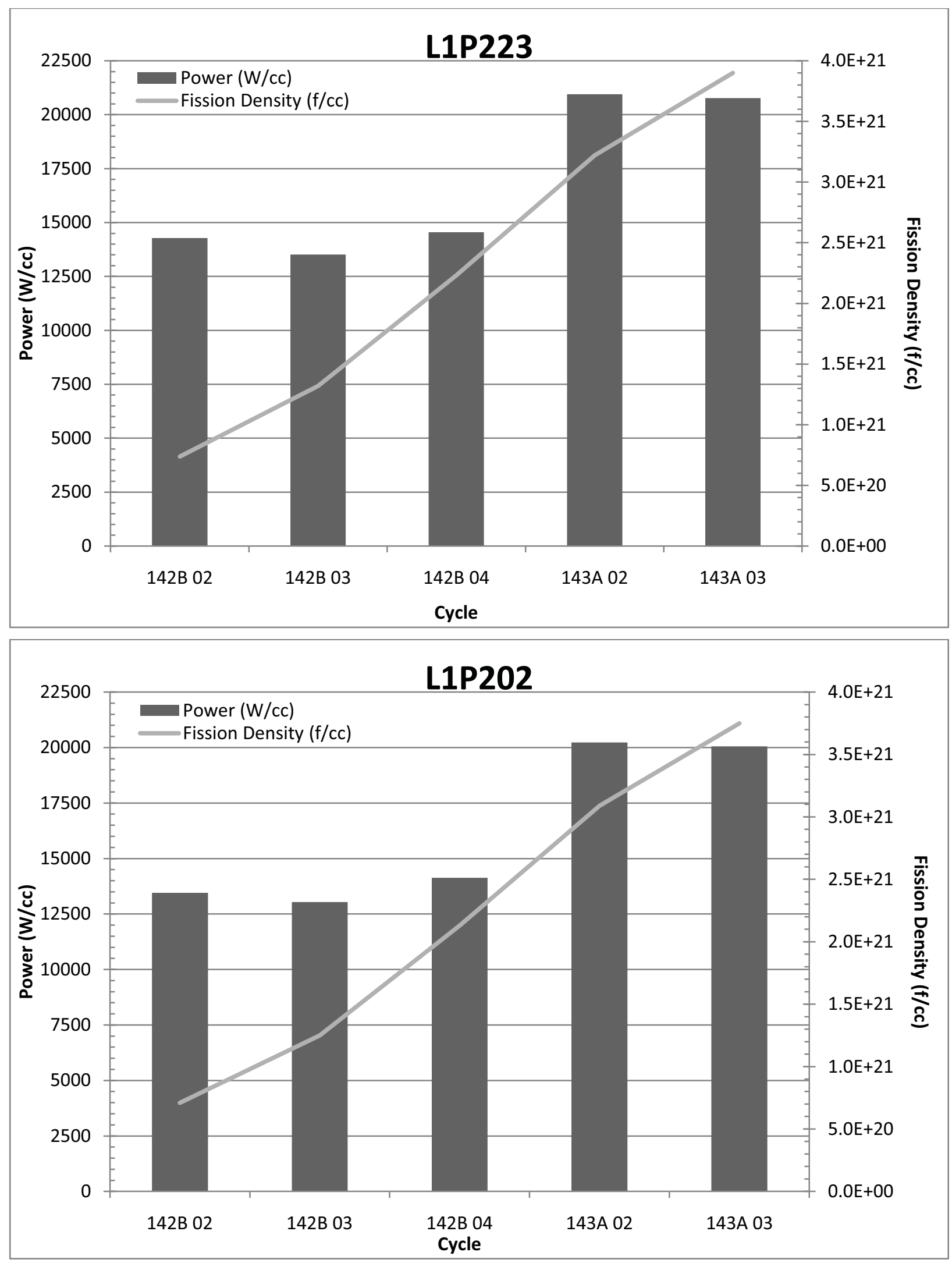


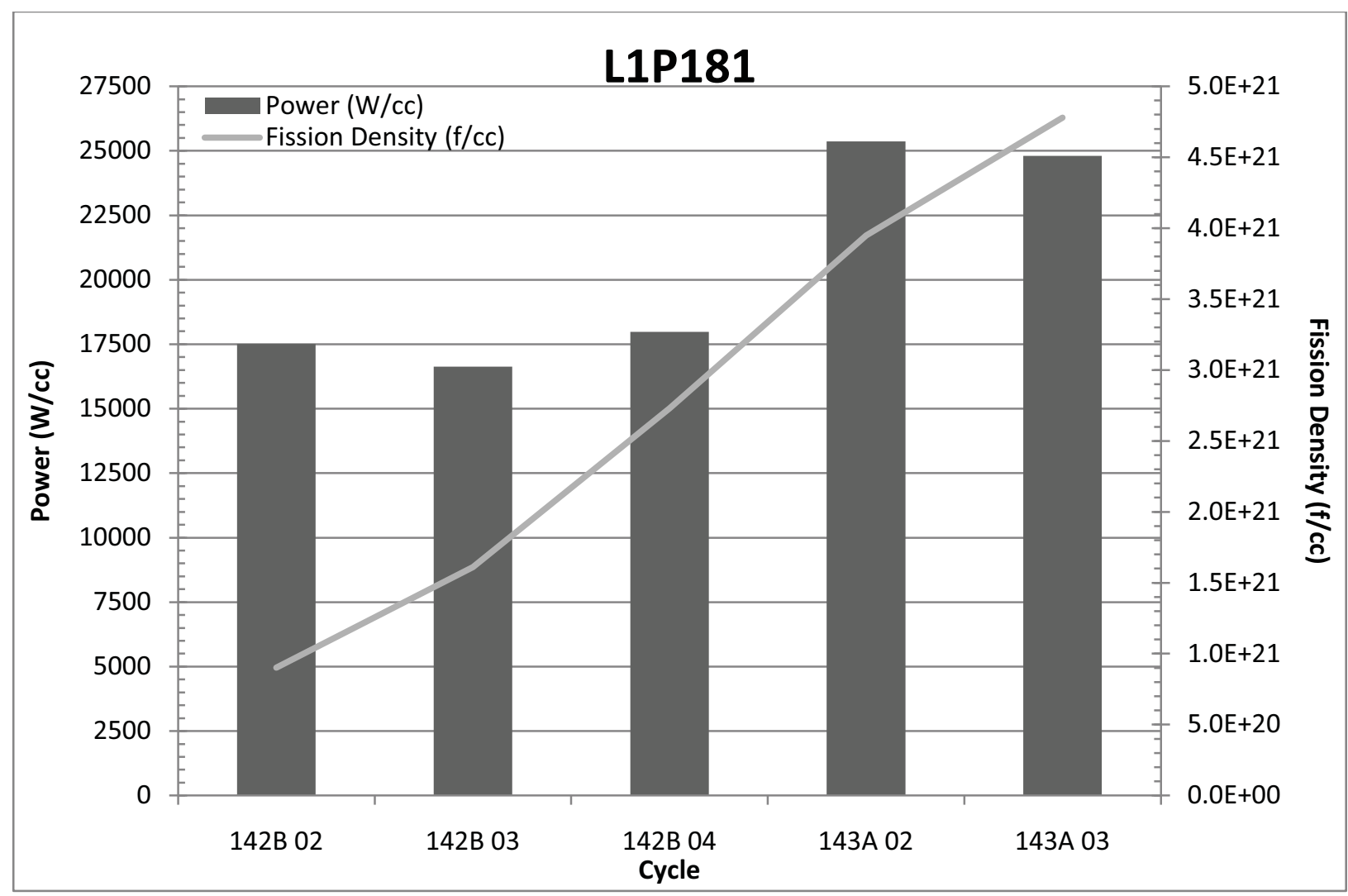

A-2. RERTR-10B

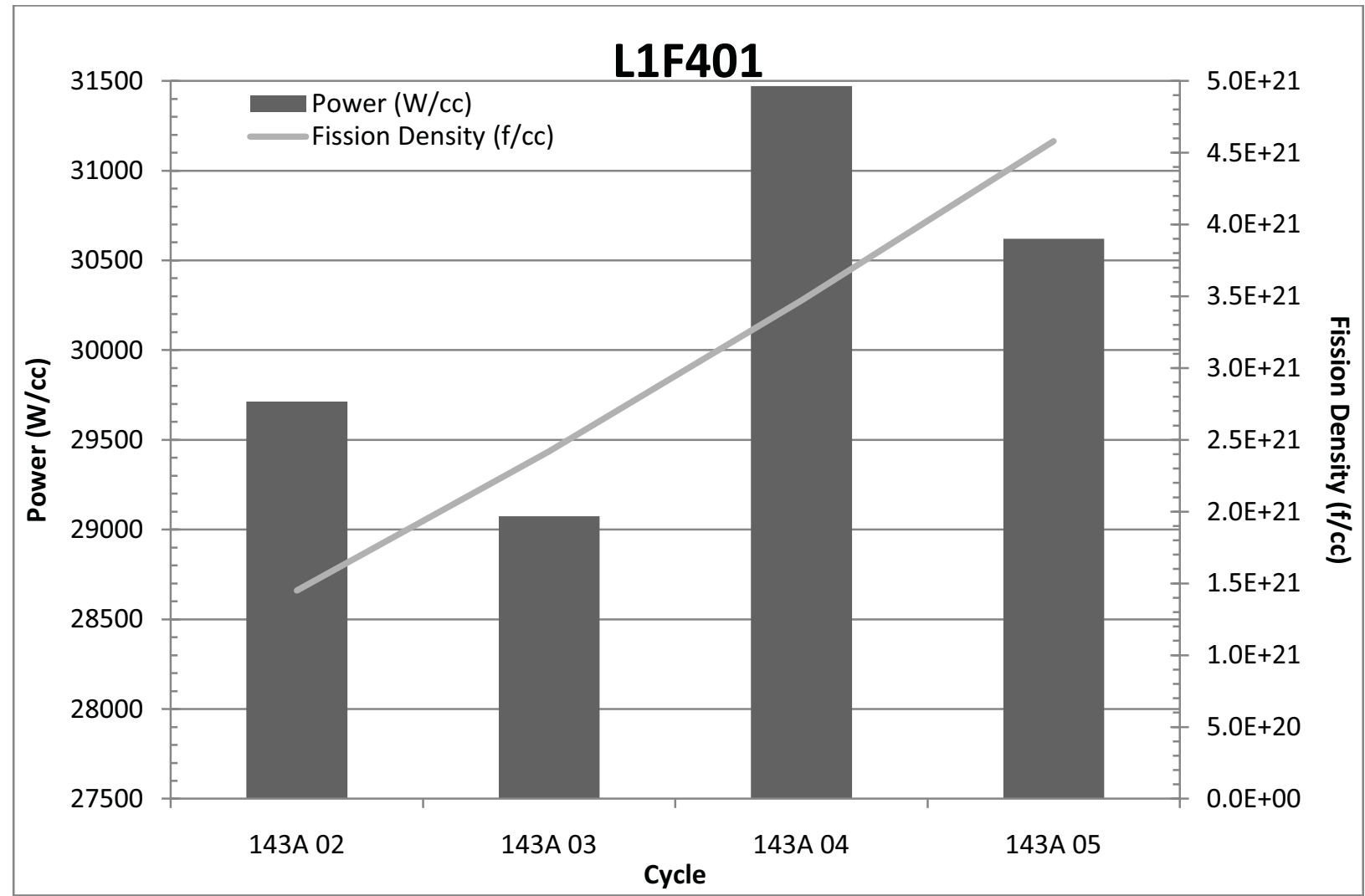



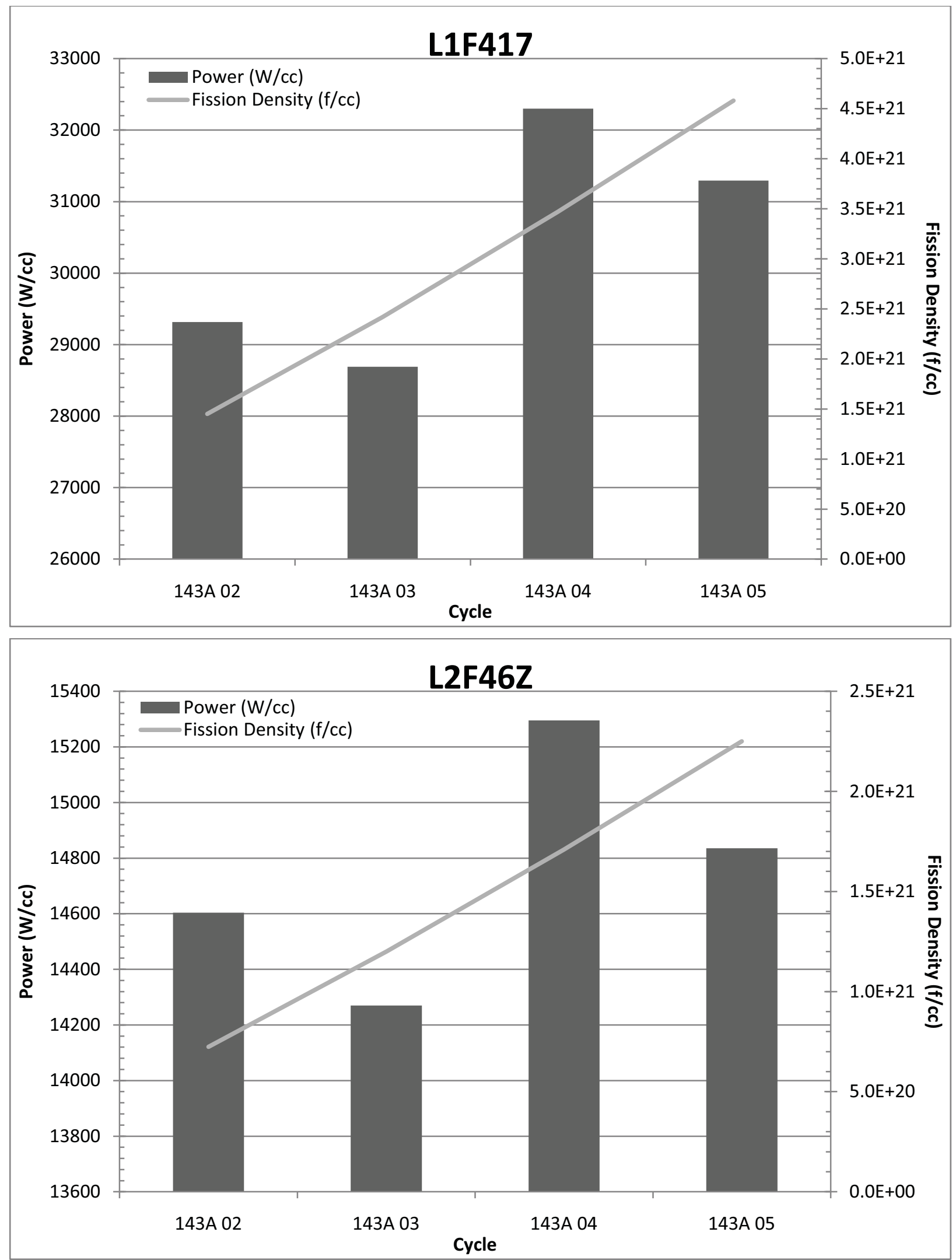

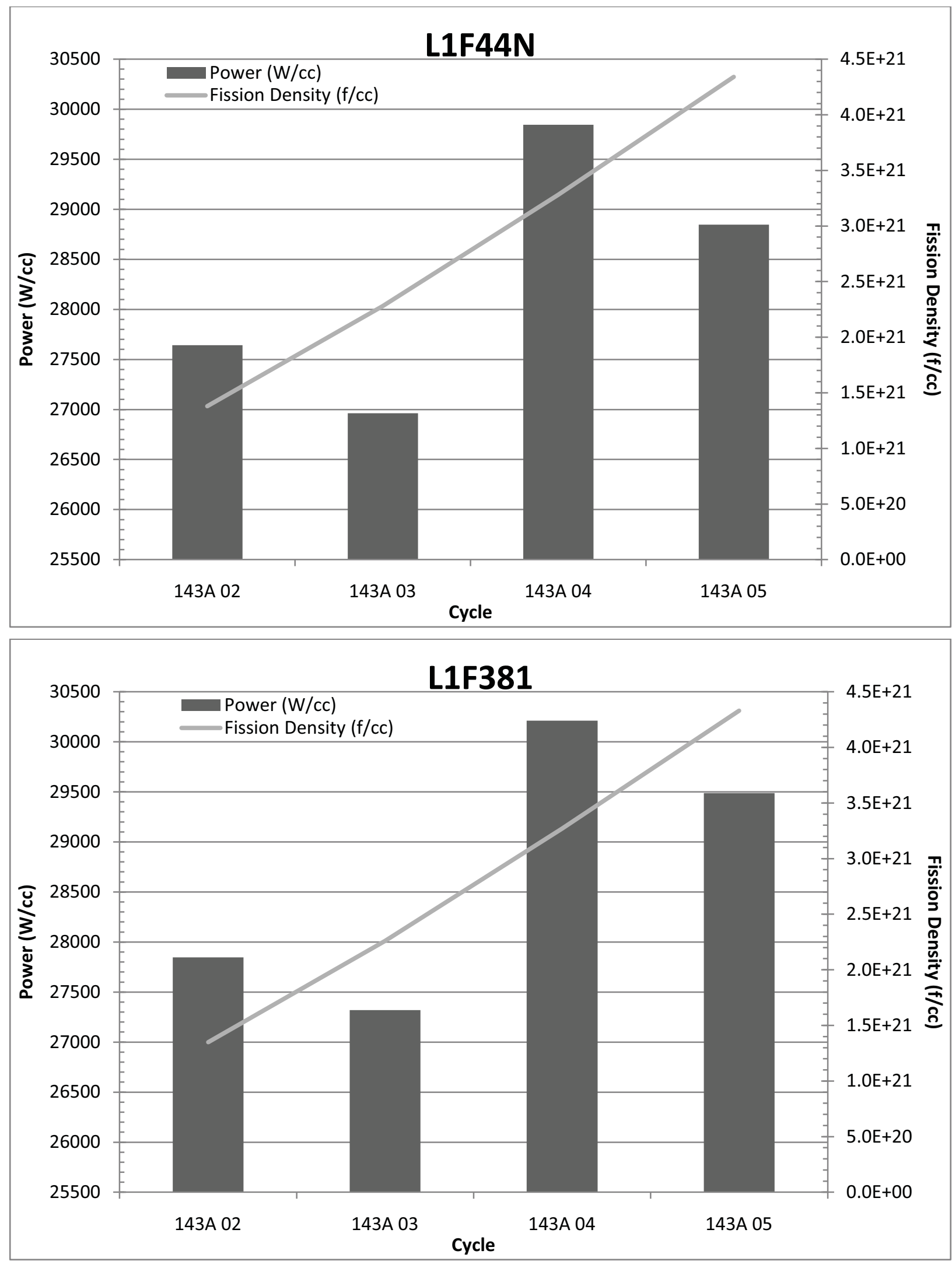

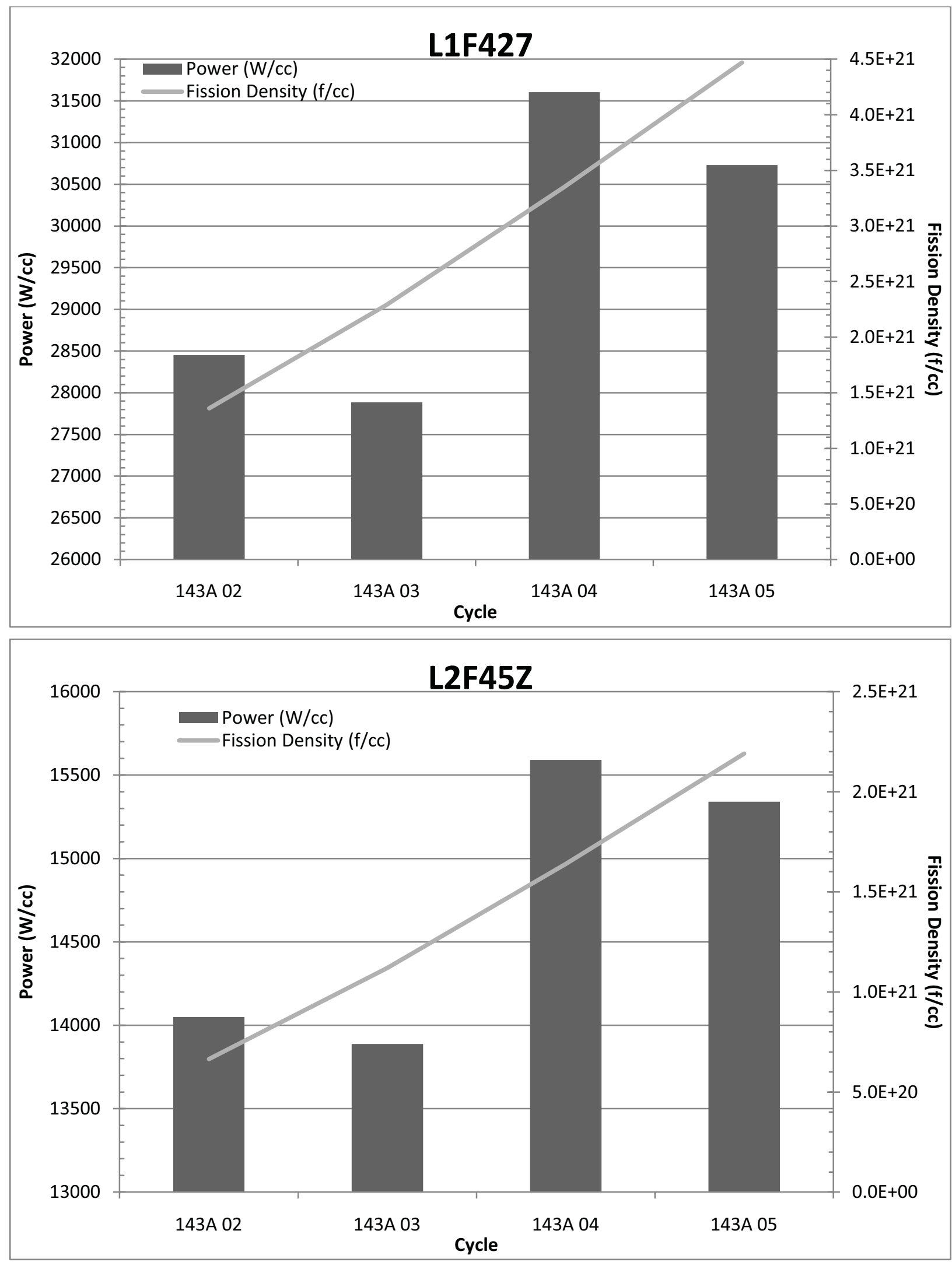


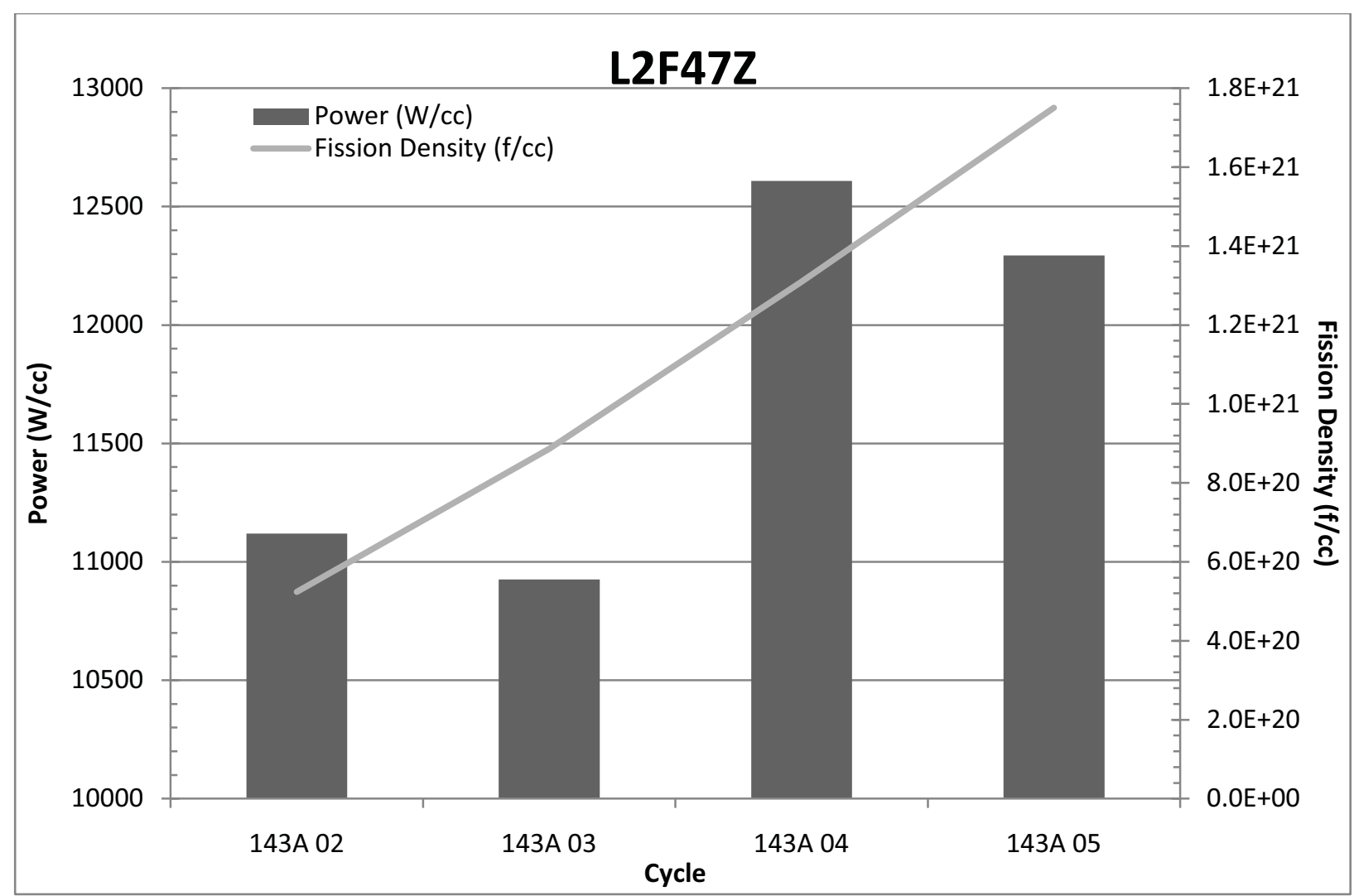


Appendix B

\section{References}




\section{Appendix B References}

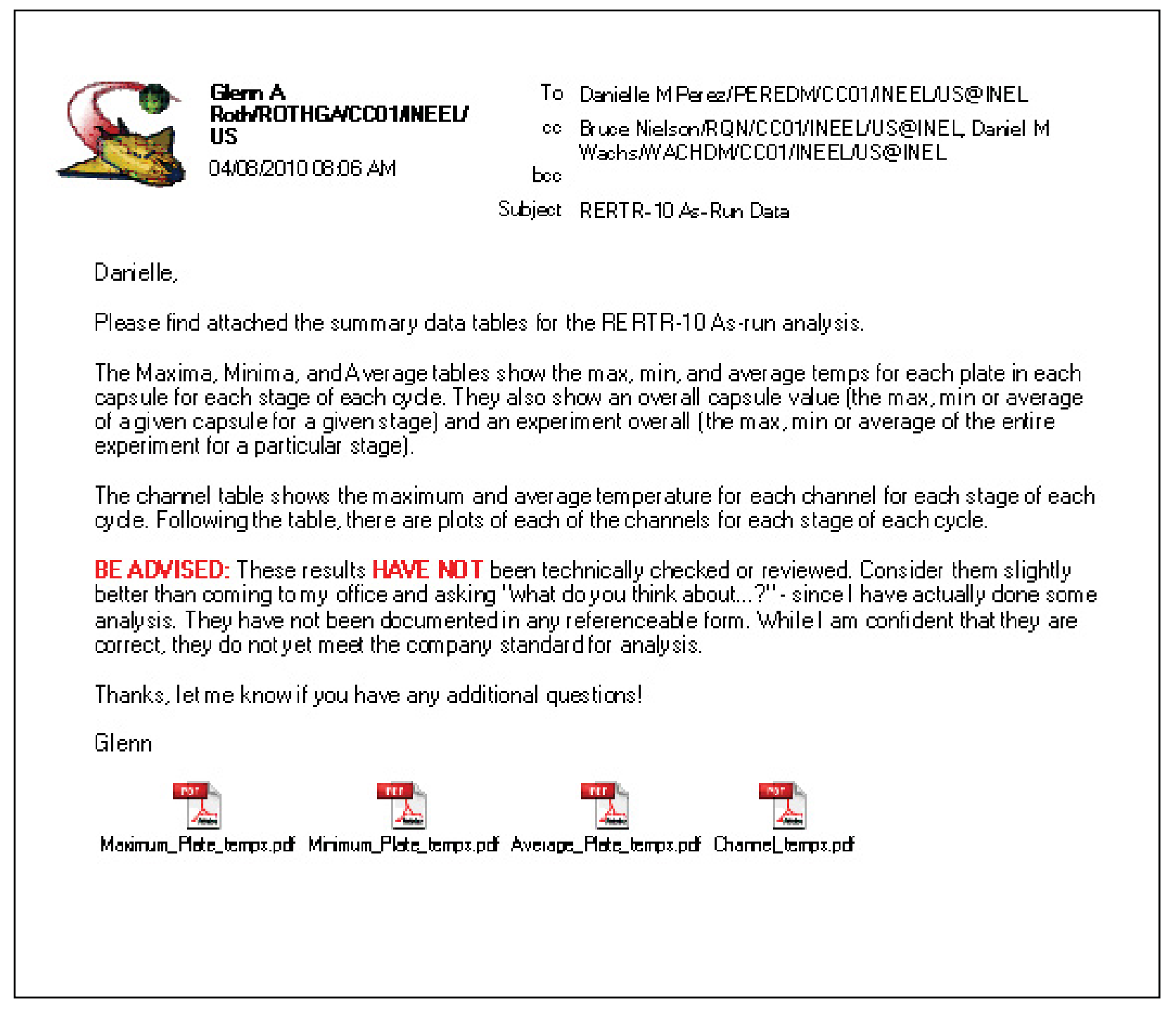




\begin{tabular}{|c|c|c|c|c|c|c|c|c|c|c|}
\hline \multirow{2}{*}{\multicolumn{2}{|c|}{ Maxima }} & \multicolumn{4}{|c|}{ Cycle 142 B } & \multicolumn{5}{|c|}{ Cycle $143 \mathrm{~A}$} \\
\hline & & 0 Day & $16 \mathrm{Da}$ & ay & 52 Day & 0 Day & 15 Day & Da & 37 Day & 48 Day \\
\hline \multirow{9}{*}{ Capsule A } & |Plate 1 & 182.1298 & 180.6166 & 178.8998 & 182.2812 & 200.224 & 197.398 & 196.58 & 128.396 & 128.396 \\
\hline & Plate 2 & 171.5048 & 170.8806 & 169.7782 & 172.9406 & 188.1538 & 186.4278 & 186.1142 & 127.8463 & 127.846 \\
\hline & Plate 3 & 171.4256 & 170.9406 & 169.6635 & 172.9179 & 188.7891 & 187.3304 & 186.9036 & 127.8473 & 127.847 \\
\hline & Plate 4 & 183.8572 & 182.9256 & 181.2234 & 185.332 & 205.8606 & 203.3462 & 202.3941 & 128.4036 & 128.4034 \\
\hline & Plate 5 & 198.55 & 196.3149 & 193.0976 & 197.6482 & 224.2854 & 220.1888 & 218.8015 & 130.4195 & 130.4105 \\
\hline & Plate 6 & 182.5624 & 180.092 & 178.4379 & 182.2788 & 202.8197 & 200.9996 & 200.4755 & 129.8597 & 8481 \\
\hline & Plate 7 & 184.2375 & 182.765 & 180.7965 & 184.8291 & 206.5679 & 203.9077 & 203.4271 & 129.8641 & 129.8521 \\
\hline & Plate 8 & 197.7725 & 195.1136 & 192.8075 & 197.4018 & 224.1447 & 219.5034 & 218.1357 & 328 & 4232 \\
\hline & Capsule & 198.55 & 196.3149 & 193.0976 & 197.6482 & 224.2854 & 220.1888 & 218.8015 & 130.4328 & 4232 \\
\hline \multirow{9}{*}{ Capsule B } & Plate 1 & 134.9549 & 134.7668 & 134.5376 & 134.947 & 142.1248 & 141.3445 & 141.1596 & 134.3482 & 134.2128 \\
\hline & Plate 2 & 134.0941 & 133.9126 & 133.7015 & 134.1222 & 246.2073 & 236.7685 & 234.8292 & 235.3138 & 232.7917 \\
\hline & Plate 3 & 134.1471 & 133.9808 & 133.7808 & 134.2212 & 248.1087 & 238.3438 & 236.3829 & 240.5374 & 237.5346 \\
\hline & Plate 4 & 135.0716 & 134.8961 & 134.6928 & 135.1367 & 142.4468 & 141.6359 & 141.445 & 134.5402 & 134.3868 \\
\hline & Plate 5 & 136.7175 & 136.5022 & 136.2707 & 136.6866 & 145.9218 & 144.7676 & 144.5186 & 123 & 137.3906 \\
\hline & Plate 6 & 135.929 & 135.7187 & 135.4975 & 135.9307 & 254.4955 & 242.7864 & 240.6173 & 240.8173 & 237.8867 \\
\hline & Plate 7 & 135.9703 & 135.7728 & 135.5591 & 136.0079 & 258.3628 & 245.9529 & 243.5834 & 246.8767 & 243.5051 \\
\hline & Plate 8 & 136. & 136.6131 & 136.3998 & 136 & 146.3646 & 1547 & 144.8923 & 137.9464 & 137.697 \\
\hline & Capsule B Overall & 136 & 136.6131 & 136.3998 & 463 & 258 & 9529 & 243.5834 & 246 & 243.5051 \\
\hline \multirow{9}{*}{ Capsule C } & Plate 1 & 213 & 205.4212 & 202.0527 & 206.3685 & 246.1824 & 237.5866 & 235.6981 & 208 & 136.0536 \\
\hline & Plate 2 & 196 & 191.0455 & 188.4007 & 192 & 232 & 225.2812 & 224.3091 & 139 & 9295 \\
\hline & Plate 3 & 194.4273 & 190.5965 & 188.0226 & 192.1141 & 231.0512 & 224.176 & 223.1784 & 139 & 9963 \\
\hline & Plate 4 & 215 & 208.8406 & 204.4709 & 209.8834 & 251.9214 & 243.5051 & 241.2953 & 329 & 136.2481 \\
\hline & Plate 5 & 215.8986 & 209.2758 & 205.148 & 1518 & 249.6578 & 4731 & 239.5386 & 179 & 1576 \\
\hline & Plate 6 & 198.9189 & 194.0969 & 191.0414 & 195.2681 & 235.8692 & 228.9346 & 227.9989 & 140.2047 & 139.9597 \\
\hline & Plate 7 & 199.7313 & 193.8526 & 191.9106 & 196.5898 & 237.0391 & 230.0528 & 229.1261 & 140.2668 & 140.0165 \\
\hline & Plate 8 & 216.5966 & 210.7375 & 207.0998 & 212.7448 & 253.7068 & 244.988 & 242.8581 & 138.5023 & 138.3274 \\
\hline & Capsule C Overall & 216.5966 & 210.7375 & 207.0998 & 212.7448 & 253.7068 & 244.988 & 242.8581 & 140.2668 & 140.0165 \\
\hline \multirow{10}{*}{ Capsule D } & Plate 1 & 145.0942 & 144.2092 & 143.6277 & 144.5073 & 161.3773 & 159.145 & 158.7012 & 142.4773 & 142.1905 \\
\hline & Plate 2 & 143.8308 & 142.9715 & 142.402 & 143.3217 & 257.2678 & 247.4263 & 245.5673 & 239.0989 & 236.7588 \\
\hline & Plate 3 & 143.9351 & 143.1267 & 142.5712 & 143.5491 & 258.0422 & 249.1101 & 247.1522 & 242.9342 & 240.1988 \\
\hline & Plate 4 & 145.3457 & 144.545 & 143.9705 & 144.9487 & 162.0436 & 159.7849 & 159.3025 & 142.8023 & 142.4869 \\
\hline & Plate 5 & 146.2847 & 145.4533 & 144.9052 & 145.7482 & 164.58 & 162.1873 & 161.7126 & 145.512 & 145.1975 \\
\hline & Plate 6 & 145.1898 & 144.3713 & 143.8283 & 144.7161 & 260.4045 & 252.1483 & 250.7797 & 246.3204 & 244.5151 \\
\hline & Plate 7 & 145.2704 & 144.4892 & 143.9559 & 144.8868 & 244.5595 & 237.3991 & 235.7698 & 231.1372 & 228.8648 \\
\hline & Plate 8 & 146.4795 & 145.7183 & 145.179 & 146.1035 & 164.6882 & 162.3058 & 161.7956 & 145.2538 & 144.9021 \\
\hline & Capsule & 146. & 145.7183 & 145.179 & 146 & 260 & 1483 & 250.7797 & 246. & 151 \\
\hline & Over all C & 216.5966 & 210.7375 & 207.0998 & 212.7448 & 260.4045 & 252.1483 & 250.7797 & 246.8767 & 244.5151 \\
\hline
\end{tabular}




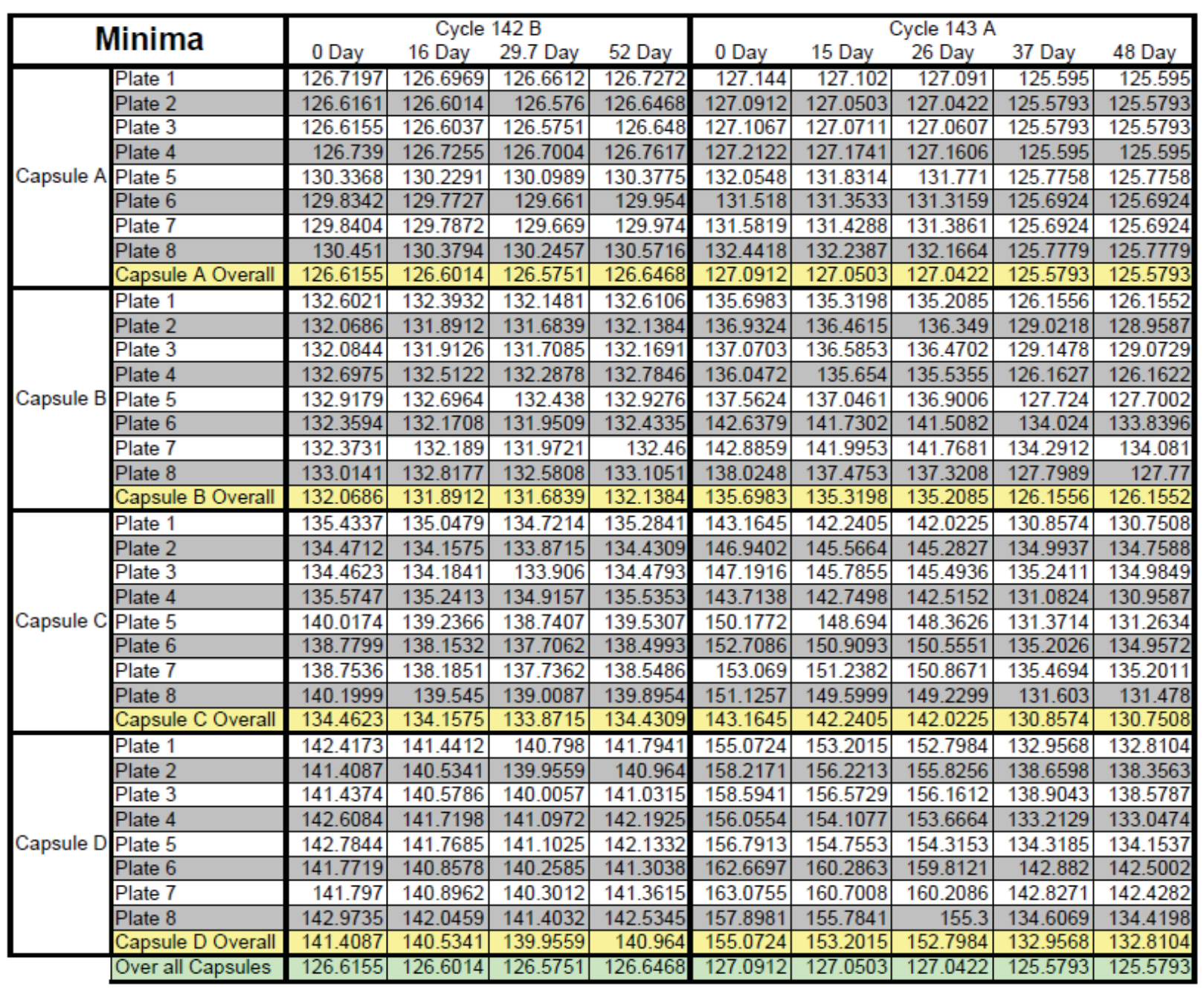




\begin{tabular}{|c|c|c|c|c|c|c|c|c|c|c|}
\hline \multirow{2}{*}{\multicolumn{2}{|c|}{ Averages }} & \multicolumn{4}{|c|}{ Cycle 142 B } & \multicolumn{5}{|c|}{ Cycle $143 \mathrm{~A}$} \\
\hline & & 0 Day & $16 \mathrm{Da}$ & Day & 52 Day & 0 Day & 15 Day & $26 \mathrm{D}$ & 37 Day & 48 Day \\
\hline \multirow{9}{*}{ Capsule A } & Plate 1 & 155.5315 & 154.7168 & 153.7754 & 155.6052 & 165.5172 & 163.9732 & 163.5243 & 126.2529 & 126.2528 \\
\hline & Plate 2 & 150.6175 & 150.2401 & 149.6255 & 151.3682 & 159.9628 & 159.0061 & 158.8265 & 126.1198 & 126.1198 \\
\hline & Plate 3 & 150.6074 & 150.3196 & 149.6097 & 151.4018 & 160.3544 & 159.5184 & 159.2816 & 126.1198 & 126.1198 \\
\hline & Plate 4 & 156.3722 & 155.842 & 154.9238 & 157.1245 & 168.384 & 166.9851 & 166.4658 & 126.2554 & 126.2552 \\
\hline & Plate 5 & 164.4787 & 163.2776 & 161.5817 & 164.0483 & 178.2951 & 176.1365 & 175.4174 & 126.7532 & 126.7528 \\
\hline & Plate 6 & 157.0072 & 155.702 & 154.7884 & 156.9306 & 168.5597 & 167.5098 & 167.2161 & 126.4472 & 126.4468 \\
\hline & Plate 7 & 157.8527 & 157.0526 & 155.9734 & 158.2181 & 170.4439 & 169.02 & 168.7454 & 126.4475 & 126.447 \\
\hline & Plate 8 & 164.1478 & 162.7633 & 161.519 & 164.0336 & 178.4344 & 176.0157 & 175.3009 & 126.7603 & 126.7599 \\
\hline & Capsule A Overall & 157.0769 & 156.2392 & 155.2246 & 157.3413 & 168.7439 & 167.2706 & 166.8472 & 126.3945 & 126.3943 \\
\hline \multirow{9}{*}{ Capsule B } & Plate 1 & 133.3737 & 133.157 & 132.8967 & 133.3694 & 137.9177 & 137.389 & 137.2458 & 128.8142 & 128.7721 \\
\hline & Plate 2 & 132.7262 & 132.5325 & 132.3079 & 132.7759 & 194.4942 & 189.1812 & 188.0855 & 185.1336 & 183.754 \\
\hline & Plate 3 & 132.7655 & 132.5849 & 132.3686 & 132.8518 & 195.6866 & 190.175 & 189.0613 & 188.0273 & 186.3771 \\
\hline & Plate 4 & 133.4914 & 133.2938 & 133.0612 & 133.5727 & 138.3186 & 137.7588 & 137.608 & 128.8939 & 128.845 \\
\hline & Plate 5 & 133.7796 & 133.5619 & 133.3014 & 133.7757 & 140.3119 & 139.5738 & 139.3922 & 588 & 130.9637 \\
\hline & Plate 6 & 133.0326 & 132.8358 & 132.6079 & 133.081 & 199.5397 & 193.0217 & 191.7972 & 188 & 8986 \\
\hline & Plate 7 & 133.0719 & 132.8877 & 132.6683 & 133.1566 & 733 & 194.819 & 193.4863 & 191 & 9922 \\
\hline & Plate 8 & 133.8978 & 133.6991 & 133.4659 & 781 & 707 & 139.9893 & 139.7974 & 131. & 1414 \\
\hline & Capsule & 133.2673 & 133.0691 & 132.8347 & 133.3201 & 168.5966 & 165.2385 & 164.5592 & 15 & 343 \\
\hline \multirow{9}{*}{ Capsule C } & Plate 1 & 175.2827 & 171.2575 & 169.3932 & 171.8478 & 008 & 192.0823 & 190.984 & 133. & 9351 \\
\hline & Plate 2 & 167.3034 & 164.4574 & 162.8978 & 763 & 19 & 187 & 186.5927 & 136. & 6613 \\
\hline & Plate 3 & 166.4668 & 164.2191 & 162.722 & 165.1881 & 191.1332 & 186.8656 & 186.2027 & 137.0992 & 136.8006 \\
\hline & Plate 4 & 176.2657 & 172.9809 & 170.6444 & 173.6617 & 200.2229 & 195.2499 & 193.9788 & 133.3226 & 133.1583 \\
\hline & Plate 5 & 177.9032 & 174.21 & 171.9335 & 174.7945 & 200.8215 & 195.8255 & 194.6773 & 133.6982 & 133.5488 \\
\hline & Plate 6 & 169.8596 & 167.0509 & 165.2514 & 167.845 & 195.3918 & 190.9355 & 190.276 & 137.1966 & 136.9118 \\
\hline & Plate 7 & 170.226 & 166.9228 & 165.6851 & 168.5125 & 196.2092 & 191.7038 & 191.0375 & 137.344 & 137.0466 \\
\hline & Plate 8 & 178.3151 & 175.0532 & 172.9915 & 176.2254 & 203.195 & 197.908 & 196.6432 & 133.9459 & 133.7771 \\
\hline & Capsule C Overall & 172.7028 & 169.519 & 167.6899 & 170.4189 & 196.9492 & 192.2258 & 191.299 & 135.3291 & 135.1049 \\
\hline \multirow{10}{*}{ Capsule D } & Plate 1 & 143.4208 & 142.4175 & 141.7609 & 142.7564 & 157.744 & 155.6249 & 155.1817 & 136.3079 & 136.1043 \\
\hline & Plate 2 & 142.2965 & 141.3751 & 140.769 & 141.78 & 209.6322 & 203.4262 & 202.2489 & 191.0701 & 189.72 \\
\hline & Plate 3 & 142.3705 & 141.4884 & 140.895 & 141.9508 & 209.9774 & 204.2509 & 203.0056 & 192.8621 & 191.2862 \\
\hline & Plate 4 & 143.6703 & 142.7633 & 142.1216 & 143.2279 & 158.6669 & 156.4854 & 156.0046 & 136.5879 & 136.361 \\
\hline & Plate 5 & 143.8145 & 142.8138 & 142.1593 & 143.1536 & 159.9477 & 157.6915 & 157.2264 & 138.5679 & 138.3298 \\
\hline & Plate 6 & 142.6164 & 141.6884 & 141.0786 & 142.0943 & 212.291 & 206.7716 & 205.8182 & 195.343 & 194.2396 \\
\hline & Plate 7 & 142.6914 & 141.8022 & 141.2045 & 142.2645 & 204.3722 & 199.4532 & 198.3554 & 187.6884 & 186.3292 \\
\hline & Plate 8 & 144.0605 & 143.1543 & 142.515 & 143.6178 & 160.6759 & 158.3815 & 157.8765 & 138.7051 & 138.4365 \\
\hline & Capsule D Overall & 143.1176 & 142.1879 & 141.563 & 142.6057 & 184.1634 & 180.2607 & 179.4647 & 164.6415 & 163.8508 \\
\hline & Over all Capsules & 151.5412 & 150.2538 & 149.328 & 150.9215 & 179.6133 & 176.2489 & 175.5425 & 146.389 & 145.9233 \\
\hline
\end{tabular}

\begin{tabular}{|c|c|c|c|c|c|c|c|c|c|c|}
\hline \multirow{2}{*}{\multicolumn{2}{|c|}{ Flow Channels }} & \multicolumn{4}{|c|}{ Cycle $142 \mathrm{~B}$} & \multicolumn{5}{|c|}{ Cycle $143 \mathrm{~A}$} \\
\hline & & 0 Day & 16 Day & 29.7 Day & 52 Day & 0 Day & 15 Day & 26 Day & 37 Day & 48 Day \\
\hline \multirow{5}{*}{$\begin{array}{c}\text { Max Temp } \\
\left({ }^{\circ} \mathrm{F}\right)\end{array}$} & Channel 1 & 144.69 & 143.44 & 142.65 & 143.76 & 157.88 & 155.72 & 155.26 & 135.29 & $\overline{135.11}$ \\
\hline & Channel 2 & 142.88 & 141.74 & 141.01 & 142.13 & 165.43 & 162.53 & 161.97 & 142.84 & 142.45 \\
\hline & Channel 3 & 141.78 & 140.8 & 140.16 & 141.29 & 174.46 & 170.81 & 170.12 & 152.69 & 152.01 \\
\hline & Channel 4 & 143.01 & 141.98 & 141.29 & 142.52 & 165.45 & 162.59 & 161.99 & 142.59 & 142.15 \\
\hline & Channel 5 & 145.21 & 144.12 & 143.32 & 144.61 & 158.85 & 156.63 & 156.12 & 135.48 & 135.26 \\
\hline \multirow{5}{*}{$\begin{array}{l}\text { Average } \\
\text { Temp }\left({ }^{\circ} \mathrm{F}\right)\end{array}$} & Channel 1 & 136.39 & 135.82 & 135.39 & 136.05 & 142.7 & 141.67 & 141.43 & 128.61 & 128.55 \\
\hline & Channel 2 & 135.68 & 135.13 & 134.72 & 135.4 & 146.53 & 145.09 & 144.79 & 132.19 & 132.01 \\
\hline & Channel 3 & 134.66 & 134.22 & 133.88 & 134.54 & 149.2239 & 147.51 & 147.18 & 135.88 & 135.59 \\
\hline & Channel 4 & 135.48 & 135.01 & 134.63 & 135.35 & 146.34 & 144.92 & 144.61 & 132.13 & 131.94 \\
\hline & Channel 5 & 136.66 & 136.15 & 135.73 & 136.49 & 143.52 & 142.44 & 142.17 & 128.74 & 128.67 \\
\hline
\end{tabular}

